\title{
Multimessengers from Core-Collapse Supernovae: Multidimensionality as a Key to Bridge Theory and Observation
}

\author{
Kei Kotake, ${ }^{1,2}$ Tomoya Takiwaki, ${ }^{2}$ Yudai Suwa, ${ }^{3}$ Wakana Iwakami Nakano, ${ }^{4}$ Shio Kawagoe, ${ }^{5}$ \\ Youhei Masada, ${ }^{6}$ and Shin-ichiro Fujimoto ${ }^{7}$ \\ ${ }^{1}$ Division of Theoretical Astronomy, National Astronomical Observatory of Japan, 2-21-1, Osawa, Mitaka, Tokyo 181-8588, Japan \\ ${ }^{2}$ Center for Computational Astrophysics, National Astronomical Observatory of Japan, Mitaka, Tokyo 181-8588, Japan \\ ${ }^{3}$ Yukawa Institute for Theoretical Physics, Kyoto University, Oiwake-cho, Kitashirakawa, Sakyo-ku, Kyoto 606-8502, Japan \\ ${ }^{4}$ Department of Aerospace Engineering, Tohoku University, 6-6-01 Aramaki-Aza-Aoba, Aoba-ku, Sendai 980-8579, Japan \\ ${ }^{5}$ Knowledge Dissemination Unit, Oshima Lab, Institute of Industrial Science, The University of Tokyo, 4-6-1 Komaba, \\ Meguro-ku, Tokyo 153-8505, Japan \\ ${ }^{6}$ Department of Computational Science, Graduate School of System Informatics, Kobe University, Nada, Kobe 657-8501, Japan \\ ${ }^{7}$ Kumamoto National College of Technology, 2659-2 Suya, Goshi, Kumamoto 861-1102, Japan \\ Correspondence should be addressed to Kei Kotake, kkotake@th.nao.ac.jp
}

Received 11 September 2011; Revised 31 January 2012; Accepted 21 March 2012

Academic Editor: Andrew Fruchter

Copyright ( 92012 Kei Kotake et al. This is an open access article distributed under the Creative Commons Attribution License, which permits unrestricted use, distribution, and reproduction in any medium, provided the original work is properly cited.

Core-collapse supernovae are dramatic explosions marking the catastrophic end of massive stars. The only means to get direct information about the supernova engine is from observations of neutrinos emitted by the forming neutron star, and through gravitational waves which are produced when the hydrodynamic flow or the neutrino flux is not perfectly spherically symmetric. The multidimensionality of the supernova engine, which breaks the sphericity of the central core such as convection, rotation, magnetic fields, and hydrodynamic instabilities of the supernova shock, is attracting great attention as the most important ingredient to understand the long-veiled explosion mechanism. Based on our recent work, we summarize properties of gravitational waves, neutrinos, and explosive nucleosynthesis obtained in a series of our multidimensional hydrodynamic simulations and discuss how the mystery of the central engines can be unraveled by deciphering these multimessengers produced under the thick veils of massive stars.

\section{Introduction}

The majority of stars more massive than $\sim 8 M_{\odot}$ end their lives as core-collapse supernovae. They have long attracted the attention of astrophysicists because they have many facets playing important roles in astrophysics. They are the mother of neutron stars as well as black holes; they play an important role for acceleration of cosmic rays; they influence galactic dynamics triggering further star formation; they are gigantic emitters of neutrinos and gravitational waves. They are also a major site for nucleosynthesis, so, naturally, any attempt to address human origins may need to begin with an understanding of core-collapse supernovae (CCSNe).

Current estimates of CCSN rates in our Galaxy predict one event every $\sim 40 \pm 10$ years [1]. When a CCSN event occurs in our galactic center, copious numbers of neutrinos are produced, some of which may be detected on the earth. Such "supernova neutrinos" will carry valuable information from deep inside the core. In fact, the detection of neutrinos from SN1987A (albeit in the Large Magellanic Cloud) opened up the Neutrino Astronomy, which is an alternative to conventional astronomy by electromagnetic waves [2,3]. Even though there were just two dozen neutrino events from SN1987A, these events have been studied extensively (yielding $\sim 500$ papers) and have allowed us to have a confidence that our basic picture of the supernova physics is correct (e.g., [4], see [5, 6] for a recent review). Recently significant progress has been made in the large water Čherenkov detectors such as Super-Kamiokande [7] and IceCube [8], and also in the liquid scintillator detector 
as KamLAND [9]. If a supernova occurs in our Galactic center $(\sim 10 \mathrm{kpc})$, about $10^{5} \bar{\nu}_{e}$ events are estimated to be detectable by IceCube (e.g., [10] and references therein). Those successful neutrino detections are important not only to study the supernova physics but also to unveil the nature of neutrinos itself such as the neutrino oscillation parameters and the mass hierarchy (e.g., [5] for a recent review).

CCSNe are now about to start even another astronomy, Gravitational-Wave Astronomy. Currently long-baseline laser interferometers such as LIGO (USA, e.g., [11]), VIRGO (Italy) (http://www.ego-gw.it/) GEO600 (Germany)(http://geo600.aei.mpg.de/), and TAMA300 (Japan, e.g., [12]) are currently operational and preparing for the first observation (see, e.g., [13] for a recent review), by which the prediction by Einstein's theory of General Relativity (GR) can be confirmed. These instruments are being updated to their Advanced status and may start taking data, possibly detecting GWs (gravitational waves) for the first time, as soon as 2015 (see [14] for a recent review). In fact, Advanced LIGO/VIRGO, which is an upgrade of the initial LIGO and VIGRO, are expected to be completed by 2015 and will increase the observable detection volume by a factor of $\sim 1000$ [15]. The Large-scale Cryogenic Gravitational-wave Telescope (LCGT [16], renamed recently as KAGRA) was funded in late 2010, which is being built under the Kamioka mine and is expected to take its first data in 2016. At such a high level of precision, those GW detectors are sensitive to many different sources, including chirp, ringdown, and merger phases of black-hole and neutron star binaries (e.g., [17-19]), neutron star normal mode oscillations (e.g., [20]), rotating neutron star mountains (e.g., [21]), and CCSN explosions (e.g., [22-24] for recent reviews), on the final of which we focus in this paper.

According to the Einstein's theory of GR (e.g., [25]), no GWs can be emitted if gravitational collapse of the supernova core proceeds perfectly spherically symmetric. To produce GWs, the gravitational collapse should proceed aspherically and dynamically. Observational evidence gathered over the last few decades has pointed towards CCSNe indeed being generally aspherical (see [26] for a recent review). The most unequivocal example is SN1987A. To explain the light-curve, a large amount of mixing of $\mathrm{Ni}$ outward to the $\mathrm{H}$-He interface and of $\mathrm{H}$ inward into the $\mathrm{He}$-core was required (e.g., Woosley [27]; Blinnikov et al. [28]; Utrobin [29], and Woosley \& Heger [30] for a review). Such mixing processes coming from the Rayleigh-Taylor instability at the interface with different compositions after shock passage have been examined extensively so far by 2D (e.g., [31-33]) and 3D simulations [34]. The asymmetry of iron and nickel lines in SN1987A was proposed to be explained, if the explosion occurs in a jet-like $[35,36]$ or in a unipolar manner [37]. More directly, the HST images of SN1987A are showing that the expanding envelope is elliptical with the long axis aligned with the rotation axis inferred from the ring ([38], see however [39] for a recent counterargument). The aspect ratio and position angle of the symmetry axis are consistent with those predicted earlier from the observations of speckle and linear polarization. What is more, the linear polarization became greater as time passed (e.g., [40-42]), a fact which has been used to argue that the central engine of the explosion is responsible for the nonsphericity (e.g., [33, 43-46]). By performing a series of time-dependent, non-LTE (local thermodynamic equilibrium), radiation-transport simulations (e.g., [47-49] , see $[50,51]$ for an alternative approach for the light-curve modeling), Dessart and Hillier [52] recently pointed out that asymmetry in the ejecta can explain the increase in the continuum polarization observed at the nebular phases $[53,54]$. Dense knots, indications of ejecta clumpiness, and filaments seen in supernova remnants by HST in the visual $[55,56]$ and by ROSAT, Chandra, and XMM-Newton [5759 ] in X-rays also provide evidence that small- and largescale inhomogeneities (and perhaps even fragmentation) are a common feature in supernova explosions.

Advancing ability of the HST has enabled the direct observation of the progenitors of nearby CCSNe from preexplosion images (see [60] for a review). Although observational measurements of the progenitor masses are currently not many and still highly uncertain (see [61] for collective references therein), evidence has accumulated that SN type II plateau (II-P) comes predominantly from stars in the range about of $8-16 M_{\odot}[60]$. A generic explosion energy of the SN II-P in the mass range is roughly on the order of $10^{51} \mathrm{erg}$ [37], however, a large diversity of the explosion signatures (i.e., explosion energy and the synthesized Ni mass) have been so far observed from quite similar progenitors [60]. For example, the inferred ${ }^{56} \mathrm{Ni}$ mass and the kinetic energies differ by a factor of five between SNe 2005cs and 2003gd, both of them are among the golden events in which enough information was obtained to give an accurate estimate on a color or spectral type of the progenitor and the initial mass. More massive stars are expected to lose much of their mass and explode as hydrogen-stripped SNe (Ib/c and IIb). Among them, the type Ic-BL SNe, which are associated with long gamma-ray bursts [62], all show much broader lines than SNe Ic. Due to the large kinetic energies of 2$5 \times 10^{52} \mathrm{erg}$, they have been referred to as "hypernovae" (e.g., [63] for a recent review). These events are likely to come from interacting binaries in which the primary exploding star has a mass lower than what is usually associated with evolution to the massive WR phase (e.g., [64-67], see however [68, 69]). In addition to the two branches mentioned above, Nomoto et al. [70] predicted yet another branch, in which the SNe II$\mathrm{P}$ with higher progenitor mass result in fainter explosions. By connecting to candidate $\mathrm{SN}$ explosion mechanisms or to progenitor structures, it is indeed best if one could obtain a unified picture to understand the mentioned wide diversity which is not only dependent on the progenitor masses but also on the evolution scenarios (a single versus binary evolution). But, this is an area for future study firstly because it is too computationally expensive to perform a long-term simulation that follows multidimensional (multiD) dynamics consistently from the onset of core-collapse, through explosion, up to the nebular phase (for example see [71] for the most up-to-date 1D modeling of spectra and light curves for binary models.), secondly because we are still inaccessible to multi-D stellar evolution models, in which multi-D modeling has been a major undertaking (see [72, 73] for recent developments). 
From a theoretical point of view, clarifying what makes the dynamics of the supernova engine deviate from spherical symmetry is essential in understanding the GW emission mechanism. Here it is worth mentioning that GWs are primary observables, which imprint a live information of the central engine, because they carry the information directly to us without being affected in propagating from the stellar center to the earth. On the other hand, SN neutrinos are exposed to a number of (external) environmental effects, including self-interaction that induces collective neutrino flavor oscillations predominantly in the vicinity of the neutrino sphere (see [74-77] for reviews of the rapidly growing research field and collective references therein) and the Mikheyev-Smirnov-Wolfenstein (MSW) effect [78] both in the stellar envelope and in the earth (see [5] for a review). Although the time profiles of neutrino signals can be potentially used like a tomography to monitor the envelope profile (e.g., [79] for a recent review), SN neutrinos generally could provide a rather indirect information about the central engine compared to GWs.

The breaking of the sphericity in the supernova engine has been considered as the most important ingredient to understand the explosion mechanism, for which supernova theorists have been continuously keeping their efforts for the past $\sim 40$ years. Currently multi-D simulations based on refined numerical models have shown several promising scenarios. Among the candidates are the neutrino heating mechanism aided by convection and hydrodynamic instabilities of the supernova shock (e.g., [80] for a review), the acoustic mechanism [81], or the magnetohydrodynamic (MHD) mechanism (e.g., [22] see references therein). To pin down the true answer among the candidate mechanisms, GW signatures, albeit being a primary observable, will not be solely enough and a careful analysis including neutrinos and photons should be indispensable. The current neutrino detectors is ready to broadcast the alert to astronomers to let them know the arrival of neutrinos (e.g., Supernova Early Warning Systems (SNEWS) [82]). In addition to optical observations using largest $8-10 \mathrm{~m}$ telescopes such as VLT and Subaru telescope (e.g., $[83,84]$ ), the planned thirty-meter telescope (TMT) (http://www.tmt.org/) with a refined spectropolarimetric technique is expected to detect more than 20 events of the SN polarization per year (Tanaka in private communication). This should provide valuable information to understand the $\mathrm{SN}$ asymmetry with increasing statistics. Not only for understanding the origin of nonspherical ejecta morphology (especially for nearby event) but more importantly for understanding the origin of heavy elements, it is of crucial importance to accurately determine nucleosynthesis in the $\mathrm{SN}$ ejecta, which naturally requires a multi-D numerical modeling (see, $[30,85]$ for as recent reviews, it is worth mentioning that radioactive decay can affect the shape of the light curve for some peculiar SN 1987A-like events [37], in which explosive nucleosynthesis plays an important role for the light-curve modeling).

Putting things together, the multidimensionality determines the explosion mechanism, in turn we may extract the information that traces the multidimensionality by the SN multimessengers, which would be only possible by a careful analysis on GWs, neutrinos, and photons. In this paper, we hope to bring together various of our published and unpublished findings from our recent multi-D supernova simulations and the obtained predictions of the SN multimessengers so far (for other high-energy astrophysical sources such as magnetars, gamma-ray bursts, and coalescing binaries, see [86-89] for recent reviews). Before we go into details from the next sections, we first have to draw a caution that the current generation of simulation results that we report in this article should depend on the next generation calculations by which more sophistication can be made not only in determining the efficiency of neutrino-matter coupling (the so-called neutrino transport calculation), but also in the treatment of general relativity. Therefore we provide here only a snapshot of the moving (long-run) documentary film whose headline we (boldly) chose to entitle as "multimessengers from CCSNe to bridge theory and observation."

Among the mentioned candidate mechanisms, we focus on the neutrino-heating mechanism in Section 2 and the MHD mechanism in Section 3, respectively. In each section, we first briefly summarize the properties of the explosion dynamics and then move on to discuss possible properties of the SN multimessengers paying particular attention to their detectability. It may be best if we can cover these SN messengers once for all in this paper, but unfortunately not. What we have studied so far is limited to GWs and explosive nucleosynthesis in the neutrino-heating mechanism, and to GWs and neutrino signals in the MHD mechanism. To compensate the uncovered fields, the related references will be given. Although a number of excellent reviews already exist on various topics in this paper, this one might go beyond such reviews by its new perspectives on the multimessenger astronomy.

\section{Neutrino-Heating Mechanism}

CCSN simulations have been counted as one of the most challenging subjects in computational astrophysics. The four fundamental forces of nature are all at play; the collapsing iron core bounces due to strong interactions; weak interactions determine the energy and lepton number loss in the core via the transport of neutrinos; electromagnetic interactions determine the properties of the stellar gas; GR plays an important role due to the compactness of the protoneutron star and also due to high velocities of the collapsing material outside. Naturally, such physical richness ranging from a microphysical scale (i.e., femto-meter scale) of strong/weak interactions to a macrophysical scale of stellar explosions has long attracted the interest of researchers, necessitating a worldwide multidisciplinary collaboration to clarify the theory of massive stellar core-collapse and the formation mechanisms of compact objects.

Ever since the first numerical simulation of such events [90], the neutrino-heating mechanism [91-93], in which a stalled bounce shock could be revived via neutrino absorption on a timescale of several hundred milliseconds 
after bounce, has been the working hypothesis of supernova theorists for these $\sim 45$ years. However, the simplest spherically-symmetric (1D) form of this mechanism fails to blow up canonical massive stars [94-97]. Pushed by mounting observations of the blast morphology (e.g., [26]) mentioned above, it is now almost certain that the breaking of the spherical symmetry is the key to solve the supernova problem. So far a number of multi-D hydrodynamic simulations have been reported, which demonstrated that hydrodynamic motions associated with convective overturn (e.g., [98-102]) as well as the Standing-Accretion-ShockInstability (SASI, e.g., [44, 103-113] see references therein) can help the onset of the neutrino-driven explosion.

To test the neutrino-heating mechanism in the multi-D context, it is of crucial importance to solve accurately the neutrino-matter coupling in spatially nonuniform hydrodynamic environments. For the purpose, one ultimately needs to solve the six-dimensional (6D) neutrino radiation transport problem (three in space, three in the momentum space of neutrinos), which is a main reason why the supernova simulations stand out from other astrophysical simulations due to their complexity. In the final sentence of the last paragraph, we wrote "demonstrated" because the neutrino heating was given by hand as an input parameter in most of the simulations cited above (see, however [101, 102]). The neutrino heating proceeds dominantly via the charged current interactions $\left(\nu_{e}+n \rightleftarrows e^{-}+p, \bar{v}_{e}+p \rightleftarrows\right.$ $\left.e^{+}+n\right)$ in the gain region. The neutrino heating rate in the gain region can be roughly expressed as $Q^{+} \propto L_{\nu}\left\langle\mu_{\nu}\right\rangle^{-1} / r^{2}$, where $L_{v}$ is the neutrino luminosity emitted from the surface of neutrino sphere and it determines the amplitude of the neutrino heating as well as cooling, and $r$ and $\left\langle\mu_{\nu}\right\rangle$ are the distance from the stellar center and the flux factor (this quantity represents the degree of anisotropy in neutrino emission; $\left\langle\mu_{\nu}\right\rangle \sim 0.25$ near at the neutrino sphere, $\left\langle\mu_{\nu}\right\rangle=1$ in the free-streaming limit $(r \rightarrow \infty))$, respectively (e.g., [114]). For example, $L_{v}$ is treated as an input parameter in the so-called "light-bulb" approach (e.g., [100]). This is one of the most prevailing approximations in recent $3 \mathrm{D}$ simulations $[111,112,115,116]$ because it is handy to study multi-D effects on the neutrino heating mechanism (albeit on the qualitative grounds). To go beyond the lightbulb scheme, $L_{v}$ should be determined in a self-consistent manner. For the purpose, one needs to tackle with neutrino transport problem, only by which energy as well as angle dependence of the neutrino distribution function can be determined without any assumptions. Since the focus of this review is on the $\mathrm{SN}$ multimessengers, a detailed discussion of various approximations and numerical techniques taken in the recent radiation-hydrodynamic SN simulations cannot be provided. Table 1 is not intended as a comprehensive compilation, but we just want to summarize milestones that have recently reported the neutrino-driven exploding models so far.

In Table 1, the first column ("Progenitor") shows the progenitor model employed in each simulation. The abbreviations of "NH," "WHW," and "WW" mean [117-119]. The second column shows SN groups with the published or submitted year of the corresponding work. "MPA" stands for the CCSN group in the Max Planck Institute for Astrophysics led by Janka and Müller. "Princeton+" stands for the group chiefly consisting of the staffs in the Princeton University (Burrows, Murphy), Caltech (Ott), Hebrew University (Livne), Université de Provence (Dessart), and their collaborators. The SN group in the Basel university is led by Liebendörfer and Thielemann. "OakRidge+" stands for the SN group mainly consisting of the Florida Atlantic University (Bruenn) and the Oak Ridge National Laboratory (Mezzacappa, Messer) and their collaborators. Tokyo+ is the SN group chiefly consisting of the staffs in the National Astronomical Observatory of Japan (myself, Takiwaki), Kyoto university (Suwa), Numazu College (Sumiyoshi), Waseda University (Yamada), and their collaborators. The third column represents the mechanism of explosions which are basically categorized into two (to date), namely by the neutrino-heating mechanism (indicated by " $\nu$-driven") or by the acoustic mechanism ("Acoustic"). "Dim." in the fourth column is the fluid space dimensions which is one-, two-, or three-dimension (1-,2-,3D). The abbreviation " $\mathrm{N}$ " stands for "Newtonian," while "PN"_for "Post-Newtonian"—_tands for some attempt at inclusion of general relativistic effects, and "GR" denotes full relativity. $t_{\exp }$ in the fifth column indicates an approximate typical timescale when the explosion initiates and $E_{\text {exp }}$ represents the explosion energy normalized by Bethe $\left(=10^{51} \mathrm{erg}\right)$ given at the postbounce time of $t_{\mathrm{pb}}$, both of which are attempted to be sought in literatures (but if we cannot find them, we remain them as blank "-"). In the final column of " $v$ transport," "Dim" represents $v$ momentum dimensions and the treatment of the velocitydependent term in the transport equations is symbolized by $\mathcal{O}(v / c)$. The definition of the "RBR," "IDSA," and "MGFLD" will be given soon in the following.

Due to the page limit of this paper, we have to start the story only after 2006 (see, e.g., [80] for a complete review, and also [120] for a similar table before 2006). The first news of the exploding model was reported by the MPA group. By performing radiation-neutrino-hydrodynamic simulations which includes one of the best available neutrino transfer approximations, they reported $1 \mathrm{D}$ and $2 \mathrm{D}$ explosions for the $8.8 M_{\odot}$ star $[121,122]$ whose progenitor has a very tenuous outer envelope with steep density gradient (a characteristic property of AGB stars). Also in 1D, the Basel+ group reported explosions for 10 and $15 M_{\odot}$ progenitors of WHW02 triggered by the hypothesised first-order QCD phase transition in the protoneutron star (PNS) [123]. To date, these two are the only modern numerical results where the neutrino-driven mechanism succeeded in 1D. In the 2D MPA simulations, they obtained explosions for a nonrotating $11.2 M_{\odot}$ progenitor of WHW02 [124], and then for a $15 M_{\odot}$ progenitor [125] of WW95 with a relatively rapid rotation imposed (by comparing the precollapse angular velocity to the one predicted in a recent stellar evolution calculation $[126,127])$. They newly brought in the socalled "ray-by-ray" approach (indicated by "RBR" in the table), in which the neutrino transport is solved along a given radial direction assuming that the hydrodynamic medium for the direction is spherically symmetric. This method, which reduces the $2 \mathrm{D}$ problem partly to $1 \mathrm{D}$, fits 
TABLE 1: Selected lists of recent neutrino-radiation hydrodynamic milestones reported by many SN groups around the world ("Group"), which obtained explosions by the neutrino-heating mechanism (indicated by " $\nu$-driven") or the acoustic mechanism ("Acoustic") (See text for more details).

\begin{tabular}{|c|c|c|c|c|c|c|}
\hline Progenitor & $\begin{array}{l}\text { Group } \\
\text { (Year) }\end{array}$ & Mechanism & $\begin{array}{c}\text { Dim. } \\
\text { (Hydro) }\end{array}$ & $\begin{array}{c}t_{\exp } \\
(\mathrm{ms})\end{array}$ & $\begin{array}{c}E_{\exp }(\mathrm{B}) \\
@ t_{\mathrm{pb}}(\mathrm{ms})\end{array}$ & $\begin{array}{c}v \text { transport } \\
(\operatorname{Dim}, \mathcal{O}(v / c))\end{array}$ \\
\hline \multirow{4}{*}{$\begin{array}{l}8.8 M_{\odot} \\
(\mathrm{NH} 88)\end{array}$} & MPA & driven & $1 \mathrm{D}(2 \mathrm{D})$ & \multirow{2}{*}{$\sim 200$} & 0.1 & Boltzmann \\
\hline & $(2006,2011)$ & $v$-ariven & $(\mathrm{PN})$ & & $(\sim 800)$ & $2, \mathcal{O}(v / c)$ \\
\hline & Princeton+ & \multirow{2}{*}{$\nu$-driven } & $2 \mathrm{D}$ & \multirow{2}{*}{$\lesssim 125$} & 0.1 & MGFLD \\
\hline & (2006) & & $(\mathrm{N})$ & & - & $1,(\mathrm{~N})$ \\
\hline \multirow{2}{*}{$\begin{array}{l}10 M_{\odot} \\
(\mathrm{WHW} 02)\end{array}$} & Basel & $v+$ & $1 \mathrm{D}$ & \multirow{2}{*}{255} & 0.44 & Boltzmann \\
\hline & $(2009)$ & (QCD transition) & (GR) & & $(350)$ & $2,(\mathrm{GR})$ \\
\hline \multirow{2}{*}{$\begin{array}{l}11 M_{\odot} \\
\text { (WW95) }\end{array}$} & Princeton+ & \multirow{2}{*}{ Acoustic } & $2 \mathrm{D}$ & \multirow{2}{*}{$\gtrsim 550$} & $\sim 0.1^{*}$ & MGFLD \\
\hline & $(2006)$ & & $(\mathrm{N})$ & & $(1000)$ & $1,(\mathrm{~N})$ \\
\hline \multirow{6}{*}{$\begin{array}{l}11.2 M_{\odot} \\
(\mathrm{WHW} 02)\end{array}$} & MPA & \multirow{2}{*}{$v$-driven } & $2 \mathrm{D}$ & $\sim 100$ & $\sim 0.005,0.025$ & “RBR” Boltz- \\
\hline & $(2006,2012)$ & & $(\mathrm{PN}, C-G R)$ & $\sim 200$ & $\sim 200,900$ & mann, $2, \mathcal{O}(v / c)$ \\
\hline & Princeton+ & \multirow[t]{2}{*}{ Acoustic } & $2 \mathrm{D}$ & \multirow{2}{*}{$\gtrsim 1100$} & $\sim 0.1^{*}$ & MGFLD \\
\hline & (2007) & & $(\mathrm{N})$ & & $(1000)$ & $1,(\mathrm{~N})$ \\
\hline & Tokyo+ & \multirow{2}{*}{$\nu$-driven } & $3 \mathrm{D}$ & \multirow[t]{2}{*}{$\sim 100$} & 0.01 & IDSA \\
\hline & $(2011)$ & & $(\mathrm{N})$ & & $(300)$ & $1,(\mathrm{~N})$ \\
\hline \multirow{2}{*}{$\begin{array}{l}12 M_{\odot} \\
(\mathrm{WHW} 02)\end{array}$} & Oak Ridge+ & \multirow{2}{*}{$\nu$-driven } & $2 \mathrm{D}$ & \multirow{2}{*}{$\sim 300$} & 0.3 & “RBR” MGFLD \\
\hline & (2009) & & $(\mathrm{PN})$ & & $(1000)$ & $1, \mathcal{O}(v / c)$ \\
\hline \multirow{4}{*}{$\begin{array}{l}13 M_{\odot} \\
(\mathrm{WHW} 02) \\
(\mathrm{NH} 88)\end{array}$} & Princeton+ & \multirow{2}{*}{ Acoustic } & $2 \mathrm{D}$ & \multirow{2}{*}{$\gtrsim 1100$} & $\sim 0.3^{*}$ & MGFLD \\
\hline & (2007) & & $(\mathrm{N})$ & & $(1400)$ & $1,(\mathrm{~N})$ \\
\hline & Tokyo+ & \multirow{2}{*}{$v$-driven } & $2 \mathrm{D}$ & \multirow{2}{*}{$\sim 200$} & 0.1 & IDSA \\
\hline & (2010) & & $(\mathrm{N})$ & & $(500)$ & $1,(\mathrm{~N})$ \\
\hline \multirow{6}{*}{$\begin{array}{l}15 M_{\odot} \\
\text { (WW95) } \\
\text { (WHW02) }\end{array}$} & MPA & \multirow{2}{*}{$\nu$-driven } & $2 \mathrm{D}$ & $\sim 600$ & $0.025,0.125$ & Boltzmann \\
\hline & $(2009,2012)$ & & $(\mathrm{PN}, C-G R)$ & $\sim 400$ & $(\sim 700,800)$ & $2, \mathcal{O}(v / c)$ \\
\hline & Princeton+ & \multirow{2}{*}{ Acoustic } & $2 \mathrm{D}$ & \multirow{2}{*}{-} & - & MGFLD \\
\hline & & & $(\mathrm{N})$ & & $(-)$ & $1,(\mathrm{~N})$ \\
\hline & Oak Ridge+ & \multirow{2}{*}{$\nu$-driven } & $2 \mathrm{D}$ & \multirow{2}{*}{$\sim 300$} & $\sim 0.3$ & “RBR” MGFLD \\
\hline & (2009) & & $(\mathrm{PN})$ & & $(600)$ & $1, \mathcal{O}(v / c)$ \\
\hline $20 M_{\odot}$ & Princeton+ & Acoustic & $2 \mathrm{D}$ & $>1200$ & $\sim 0.7^{*}$ & MGFLD \\
\hline (WHW02) & (2007) & & $(\mathrm{N})$ & & $(1400)$ & $1,(\mathrm{~N})$ \\
\hline & Princeton+ & Acoustic & $2 \mathrm{D}$ & $>1200$ & - & MGFLD \\
\hline $25 M_{\odot}$ & (2007) & Acoustic & $(\mathrm{N})$ & z & $(-)$ & $1,(\mathrm{~N})$ \\
\hline (WHW02) & Oak Ridge+ & $\nu$-driven & $2 \mathrm{D}$ & $\sim 300$ & $\sim 0.7$ & “RBR” MGFLD \\
\hline & (2009) & $v$-ariven & $(\mathrm{PN})$ & & (1200) & $1, \mathcal{O}(v / c)$ \\
\hline
\end{tabular}

well with their original 1D Boltzmann solver [94] (note that the ray-by-ray approach has an advantage compared to other approximation schemes, such that it can fully take into account the available neutrino reactions (e.g., [128] for references therein) and also give us the most accurate solution for a given angular direction). For 2D hydrodynamic simulations with the ray-by-ray transport, one needs to solve the $4 \mathrm{D}$ radiation transport problem (two in space and two in the neutrino momentum space). Regarding the explosion energies obtained in the MPA simulations, their values at their final simulation time are typically underpowered by one or two orders of magnitudes to explain the canonical supernova kinetic energy $\left(\sim 10^{51} \mathrm{erg}\right)$. But the explosion energies presented in their figures are still growing with time, and they could be as high as $1 \mathrm{~B}$ if they were able to follow a much longer evolution as discussed in [124]. Very recently, Mueller et al. [129] reported that more energetic explosions are obtained for the 11.2 and $15 M_{\odot}$ stars in their GR 2D simulations compared to the corresponding post-Newtonian models (e.g., [125]). In the table, the data in italic character represent their most up-to-date results based on the 2D GR simulations using conformally-flatness approximation (e.g., $[130,131]$, indicated by " $C-G R$ " in the table).

It is rather only recently that fully $2 \mathrm{D}$ multiangle Boltzmann transport simulations become practicable by the Princeton+ group $[132,133]$. In this case, one needs to handle the $5 \mathrm{D}$ problem for $2 \mathrm{D}$ simulations (two in space, and three in the neutrino momentum space). However 
this scheme is very computationally expensive currently to perform long-term supernova simulations. In fact, the most recent 2D work by Brandt et al. [133] succeeded in following the dynamics until $\sim 400 \mathrm{~ms}$ after bounce for a nonrotating and a rapidly rotating $20 M_{\odot}$ model of WHW02, but explosions seemingly have not been obtained in such an earlier phase either by the neutrino-heating or the acoustic mechanism.

In the table, "MGFLD" stands for the multigroup Flux-limited diffusion scheme which eliminates the angular dependence of the neutrino distribution function (see, e.g., [134] for more details). For 2D simulations, one needs to solve the $3 \mathrm{D}$ problem, namely two in space, and one in the neutrino momentum space. By implementing the MGFLD algorithm to the CHIMERA code in a ray-by-ray fashion (e.g., [135]), Bruenn et al. (2009) obtained neutrino-driven explosions for nonrotating progenitors in a relatively wide range in $12,15,20,25 M_{\odot}$ of WHW02 (see Table 1). These models tend to start exploding at around $300 \mathrm{~m}$ after bounce, and the explosion energy for the longest running model of the $25 M_{\odot}$ progenitor is reaching to $1 \mathrm{~B}$ at $1.2 \mathrm{~s}$ after bounce [135].

On the other hand, the 2D MGFLD simulations implemented in the VULCAN code [136] obtained explosions for a variety of progenitors of $11,11.2,13,15,20$, and $25 M_{\odot}$ not by the neutrino-heating mechanism but by the acoustic mechanism (for the $8.8 M_{\odot}$ progenitor, they obtained neutrinodriven explosions (see Table 1)). The acoustic mechanism relies on the revival of the stalled bounce shock by the energy deposition via the acoustic waves that the oscillating protoneutron stars (PNSs) would emit in a much delayed phase ( $\sim 1$ second) compared to the conventional neutrinoheating mechanism ( 300-600 milliseconds). If the core pulsation energy given in Burrows et al. [45] could be used to measure the explosion energy in the acoustic mechanism, they reach to $1 \mathrm{~B}$ after $1000 \mathrm{~ms}$ after bounce.

By performing 2D simulations in which the spectral neutrino transport was solved by the isotropic diffusion source approximation (IDSA) scheme [137], the Tokyo+ group reported explosions for a nonrotating and rapidly rotating $13 M_{\odot}$ progenitor of $\mathrm{NH} 88$. They pointed out that a stronger explosion is obtained for the rotating model comparing to the corresponding nonrotating model. The IDSA scheme splits the neutrino distribution into two components (namely the streaming and trapped neutrinos), both of which are solved using separate numerical techniques (see [137] for more details). The approximation level of the IDSA scheme is basically the same as the one of the MGFLD. The main advantage of the IDSA scheme is that the fluxes in the transparent region can be determined by the nonlocal distribution of sources rather than the gradient of the local intensity like in MGFLD. A drawback in the current version of the IDSA scheme is that heavy lepton neutrinos $\left(v_{x}\right.$, i.e., $v_{\mu}, v_{\tau}$ and their antiparticles) as well as the energy-coupling weak interactions have yet to be implemented. Extending the 2D modules in [138] to 3D, they recently reported explosions in the $3 \mathrm{D}$ models for an $11.2 M_{\odot}$ progenitor of WHW02 [139]. By comparing the convective motions as well as neutrino luminosities and

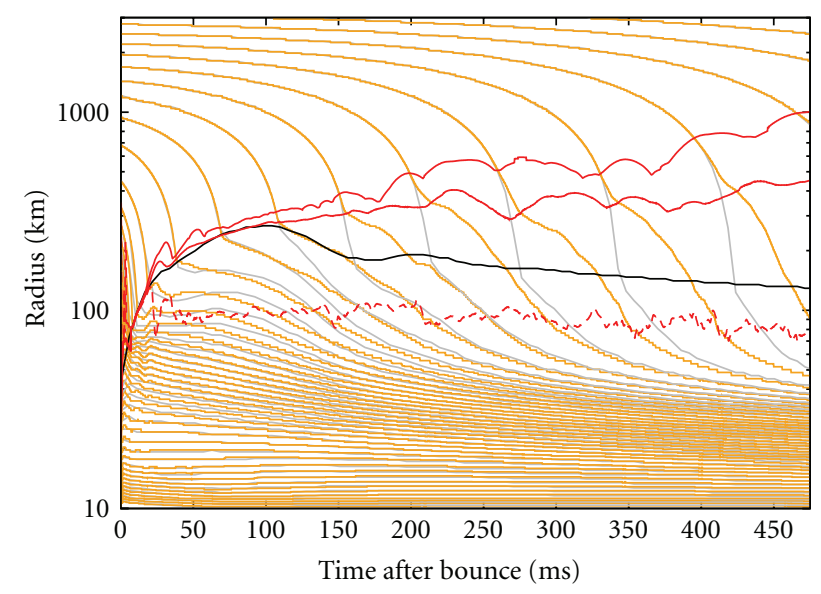

Figure 1: Time evolution of 1D (thin gray lines) or 2D (thin orange lines) hydrodynamic simulation [138] of a $13 M_{\odot}$ progenitor [117]. Thick lines in red (for 2D) and black (for 1D) show the position of shock waves, noting for $2 \mathrm{D}$ that the maximum (top) and average (bottom) shock position are shown. The red-dashed line represents the position of the gain radius, which is similar to the 1D case (not shown).

energies between their 2D and 3D models, they pointed out whether 3D effects would help explosions or not is sensitive to the employed numerical resolutions. They argued that next-generation supercomputers are at least needed to draw a robust conclusion of the $3 \mathrm{D}$ effects.

Having summarized a status of the current supernova simulations, one might easily see a number of issues that remain to be clarified. First of all, the employed progenitors usually rather scatter (e.g., Table 1). Different SN groups seem to have a tendency to employ different progenitors, providing different results. By climbing over a wall which may have rather separated exchanges among the groups, a detailed comparison for a given progenitor needs to be done seriously in the multi-D results (as have been conducted in the Boltzmann 1D simulations between the MPA, Basel+, and Oak Ridge+ groups [140]).

In the next section, we briefly summarize the findings obtained in our 2D [138] and 3D [139] simulations, paying particular attention to how multidimensionality such as SASI, convection, and rotation could affect the neutrinodriven explosions.

\subsection{Multidimensionality in Multi-D Radiation Hydrodynamic} Simulations. Figure 1 depicts the difference between the time evolutions of $1 \mathrm{D}$ (thin gray lines) or 2D (thin orange lines) simulation of the $13 M_{\odot}$ progenitor model. Until $\sim 100 \mathrm{~ms}$ after bounce, the shock position of the 2D model (thick red line) is similar to the 1D model (thick black line). Later on, however, the shock for $2 \mathrm{D}$ does not recede as for $1 \mathrm{D}$, but gradually expands and reaches $1000 \mathrm{~km}$ at about $470 \mathrm{~ms}$ after bounce. Comparing the position of the gain radius (reddashed line) to the shock position for 1D (thick black line) and 2D (thick red line), one can see that the advection time of the accreting material in the gain region can be longer in $2 \mathrm{D}$ 


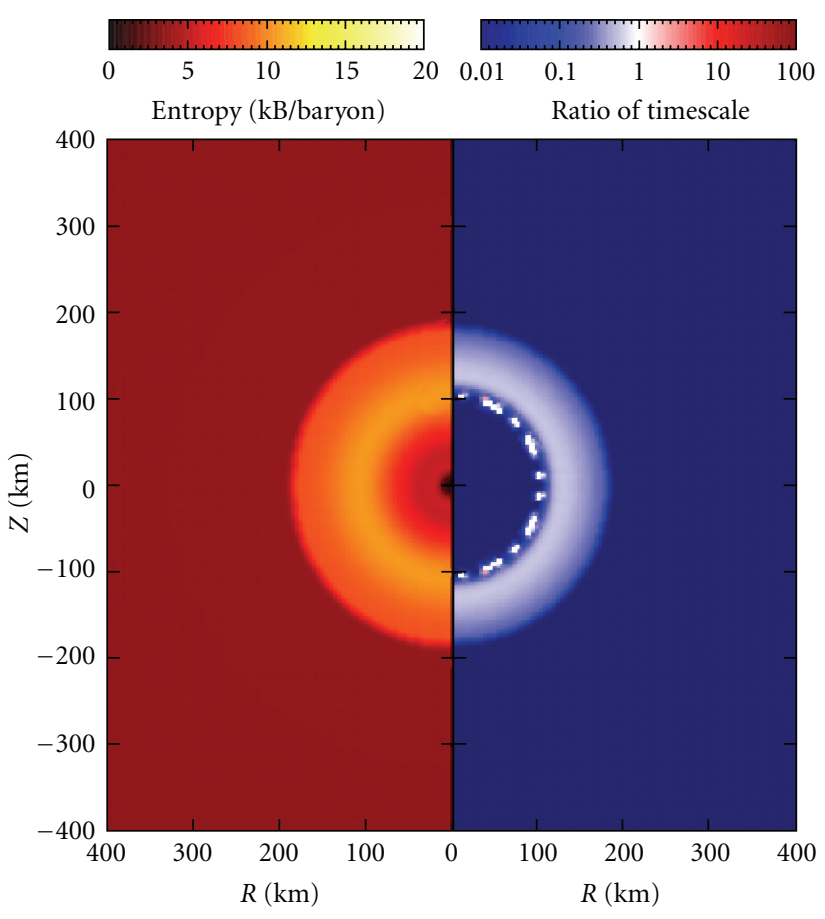

(a)

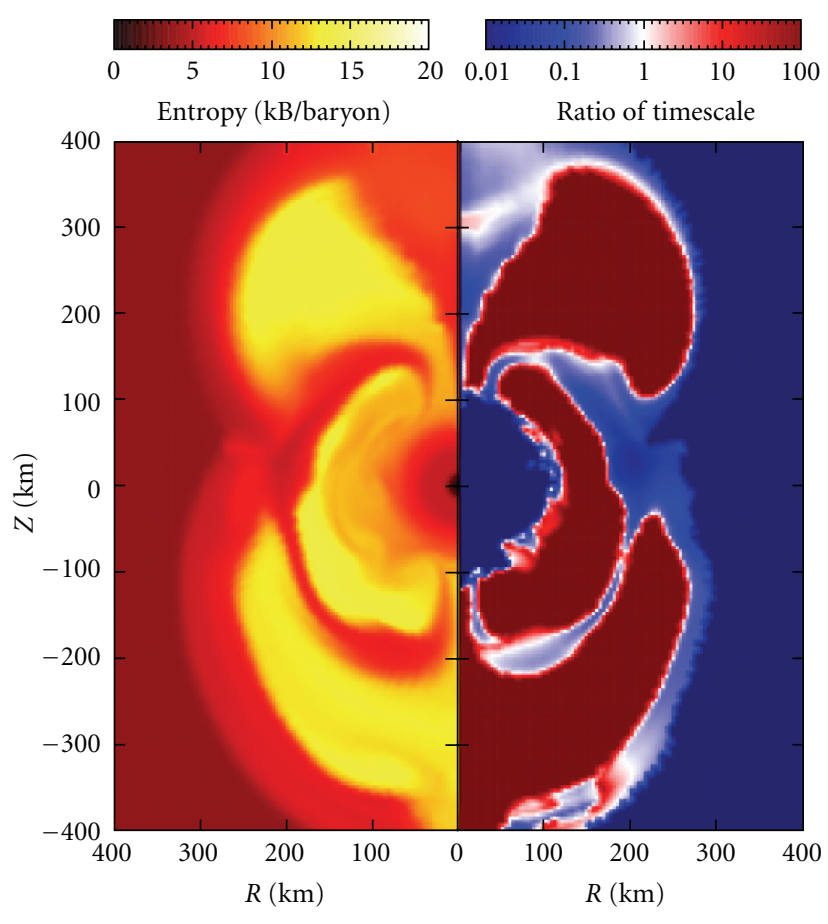

(b)

FIGURE 2: Snapshot of the distribution of entropy (left half) and the ratio of the advection to the heating timescale (right half) for models of the $1 \mathrm{D}$ (left) and 2D (right) models at $200 \mathrm{~ms}$ after bounce. These figures are taken from [138].

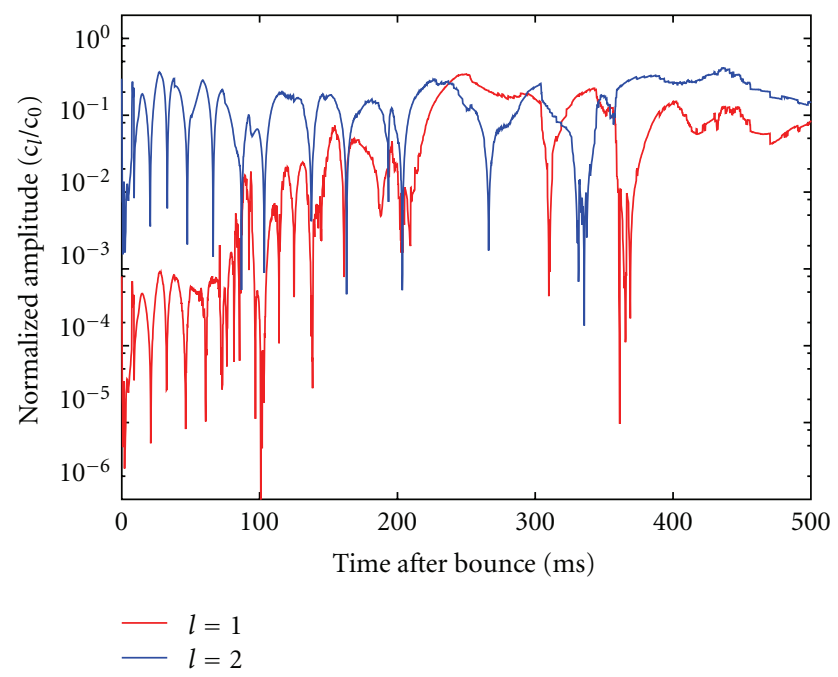

FIGURE 3: SASI activity for the rotating model versus postbounce time. Shown are the coefficients of the dipole $(\ell=1)$ and quadrupole $(\ell=2)$ modes of the spherical harmonics of the aspherical shock position, normalized to the amplitude of the $\ell=0$ mode (taken from [138]).

than in 1D. This longer exposure of cool matter in the heating region to the irradiation of hot outstreaming neutrinos from the PNS is essential for the increased efficiency of the neutrino heating in multi-D models. This can be also depicted in Figure 2 . The right half shows $\tau_{\text {adv }} / \tau_{\text {heat }}$, which is the ratio of the advection to the neutrino heating timescale. This quantity is known as a useful quantity to diagnose the success $\left(\tau_{\text {adv }} / \tau_{\text {heat }} \gtrsim 1\right.$, i.e., the neutrino-heating timescale is shorter than the advection timescale of material in the gain region) or failure $\left(\tau_{\mathrm{adv}} / \tau_{\text {heat }} \lesssim 1\right)$ of the neutrino-driven explosion (e.g., [141]). For the 2D model (right panel), it can be shown that the condition of $\tau_{\text {adv }} / \tau_{\text {heat }} \gtrsim 1$ is satisfied behind the aspherical shock, which is deformed predominantly by the SASI ( $\ell=2$ mode at the snapshot), while the ratio is shown to be smaller than unity in the whole region behind the spherical standing accretion shock (left panel: 1D). Here SASI that develops due to the advectionacoustic cycle in the supernova core $[142,143]$, is a uni- and bipolar sloshing of the stalled bounce shock with pulsational strong expansion and contraction (seen as oscillations in the red curves in Figure 1). Comparing the left half of each panel, the entropy for the 2D model is shown to be larger than for the $1 \mathrm{D}$ model. This is also the evidence that the neutrino heating works more efficiently in 2D.

To see clearly the effects of stellar rotation on the postbounce evolution, a rapidly rotating model was taken in [138], in which a constant angular frequency of $\Omega_{0}=$ $2 \mathrm{rad} / \mathrm{s}$ is imposed inside the iron core with a dipolar cut off $\left(\propto r^{-2}\right)$ outside. This corresponds to $\beta \sim 0.18 \%$ with $\beta$ being the ratio of the rotational to the gravitational energy when the simulation starts. For the rotating model, the dominant mode of the shock deformation after bounce is almost always the $\ell=2$ mode for the rotating model (e.g., Figure 3, although the $\ell=1$ mode can be as large as the $\ell=2$ mode when the SASI enters the nonlinear regime $(\gtrsim 200 \mathrm{~ms}$ 
after bounce). In contrast to this rotation-induced $\ell=2$ deformation, the $\ell=1$ mode tends to be larger than the $\ell=2$ mode for the $2 \mathrm{D}$ models without rotation in the saturation phase.

The top panel of Figure 4 shows the comparison of the explosion energies for the $2 \mathrm{D}$ models with and without rotation (models with “-rot" or “-2D," resp.). Although the explosion energies depend on the numerical resolutions quantitatively, they show a continuous increase for the rotating models. The explosion energies for the models without rotation, on the other hand, peak around $180 \mathrm{~ms}$ when the neutrino-driven explosion sets in (see also Figure 1), and show a decrease later on. The reason for the greater explosion energy for models with rotation is due to the bigger mass of the exploding material. This is because the northsouth symmetric $(\ell=2)$ explosion can expel more material than for the unipolar explosion (compare the left and right of the bottom panel in Figure 4). The explosion energies when we terminated the simulation are less than $\lesssim 10^{50}$ erg for all the computed models. For the rotating models, we are tempted to speculate that the explosion energies could increase later on by a linear extrapolation. However, in order to identify the robust feature of an explosion, a longer-term simulation with improved input physics is needed. In combination with the assumed rapid rotation, magnetic fields should be also taken into account as in [46], which we are going to study as a followup of our rotating $2 \mathrm{D}$ models (Suwa et al. in preparation).

Extending our 2D modules mentioned above, we are currently running $3 \mathrm{D}$ simulations for an $11.2 M_{\odot}$ star of Woosley et al. [118] with spectral neutrino transport that is solved by the IDSA scheme in a ray-by-ray manner [139]. We briefly summarize the results in the following.

The left panel of Figure 5 shows mass-shell trajectories for the 3D (red lines) and 1D model (green line), respectively. At around $300 \mathrm{~ms}$ after bounce, the average shock radius for the 3D model exceeds $1000 \mathrm{~km}$ in radius. On the other hand, an explosion is not obtained for the 1D model, which is in agreement with Buras et al. [124]. The right panel of Figure 5 shows a comparison of the average shock radius versus postbounce time. In the $2 \mathrm{D}$ model, the shock expands rather continuously after bounce. This trend is qualitatively consistent with the 2D result by Buras et al. [124] (see their Figure 15 for model s112_128_f). The reason that the average shock of our 2D model expands much faster than theirs would come from the neglected effects in this work including general relativistic effects, inelastic neutrinoelectron scattering, and cooling by heavy-lepton neutrinos. All of them could give a more optimistic condition to produce explosions. Apparently these ingredients should be appropriately implemented, which we hope to be practicable in the next-generation 3D simulations.

Comparing the shock evolution between our 2D (green line in the right panel of Figure 5) and 3D models (red line), the shock is shown to expand much faster for $2 \mathrm{D}$. The pink line labeled by "3D low" is for the low resolution $3 \mathrm{D}$ model, in which the mesh numbers are taken to be half of the standard model. Note that the 3D computational grid consists of 300 logarithmically spaced, radial zones to cover from the center up to $5000 \mathrm{~km}$ and 64 polar $(\theta)$ and 32 azimuthal $(\phi)$ uniform mesh points, which are used to cover the whole solid angle. The low resolution $3 \mathrm{D}$ model has onehalf of the mesh numbers in the $\phi$ direction $\left(n_{\phi}=16\right)$, while fixing the mesh numbers in other directions. Comparing with our standard 3D model (red line), the shock expansion becomes less energetic for the low resolution model (later than $\sim 150 \mathrm{~ms}$ ). Above results indicate that explosions are easiest to obtain in $2 \mathrm{D}$, followed in order by $3 \mathrm{D}$, and $3 \mathrm{D}$ (low). At first sight, this may look contradicted with the finding by Nordhaus et al. [116] who pointed out that explosions could be more easily obtained in $3 \mathrm{D}$ than in $2 \mathrm{D}$. The reason of the discrepancy is summarized shortly as it follows.

Figure 6 compares the blast morphology for our 3D (left panel) and 2D (right) model (note that the polar axis is tilted (about $\pi / 4$ ) both in the left and middle panel). In the 3D model (left panel), nonaxisymmetric structures are clearly seen. By performing a tracer-particle analysis, the maximum residency time of material in the gain region is shown to be longer for $3 \mathrm{D}$ than $2 \mathrm{D}$ due to the nonaxisymmetric flow motions (see Figure 7). This is one of advantageous aspects of $3 \mathrm{D}$ models to obtain the neutrino-driven explosions. On the other hand, our detailed analysis showed that convective matter motions below the gain radius become much more violent in $3 \mathrm{D}$ than in $2 \mathrm{D}$, making the neutrino luminosity larger for 3D (see [139] for more details). Nevertheless the emitted neutrino energies are made smaller due to the enhanced cooling. Due to these competing ingredients, the neutrino-heating timescale becomes shorter for the $3 \mathrm{D}$ model, leading to a smaller net-heating rate compared to the corresponding 2D model (Figure 8). Note here that the spectral IDSA scheme, by which the feedback between the mass accretion and the neutrino luminosity can be treated in a self-consistent manner (not like the light-bulb scheme assuming a constant luminosity), sounds quite efficient in the first-generation 3D simulations.

As seen from Figure 5, an encouraging finding in [139] was that the shock expansion tends to become more energetic for models with finer resolutions. These results would indicate whether these advantages for driving $3 \mathrm{D}$ explosions can or cannot overwhelm the disadvantages is sensitive to the employed numerical resolutions (It is of crucial importance to conduct a convergence test in which a numerical gridding is changed in a systematic way (e.g., [144]).) To draw a robust conclusion, 3D simulations with much more higher numerical resolutions and also with more advanced treatment of neutrino transport as well as of gravity are needed, which could be hopefully practicable by utilizing forthcoming petaflops-class supercomputers.

In addition to the $3 \mathrm{D}$ effects, impacts of $\mathrm{GR}$ on the neutrino-driven mechanism stand out among the biggest open questions in the supernova theory. It should be remembered that using newly derived Einstein equations [145], the consideration of GR was standard in the pioneering era of supernova simulations (e.g., [146]). One year after Colgate and White [90], Schwartz [147] reported the first fully GR simulation of stellar collapse to study the supernova mechanism, who implemented a gray transport of neutrino 


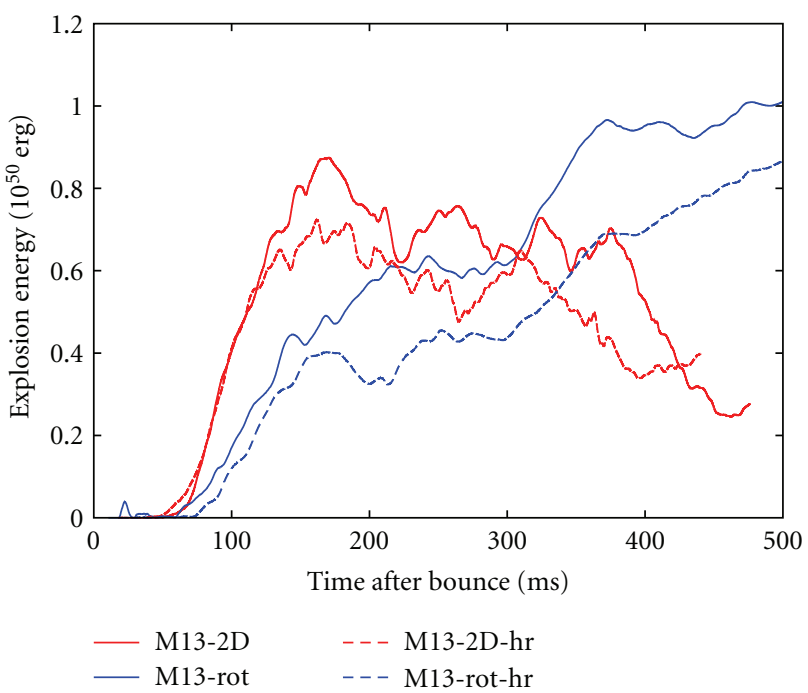

(a)
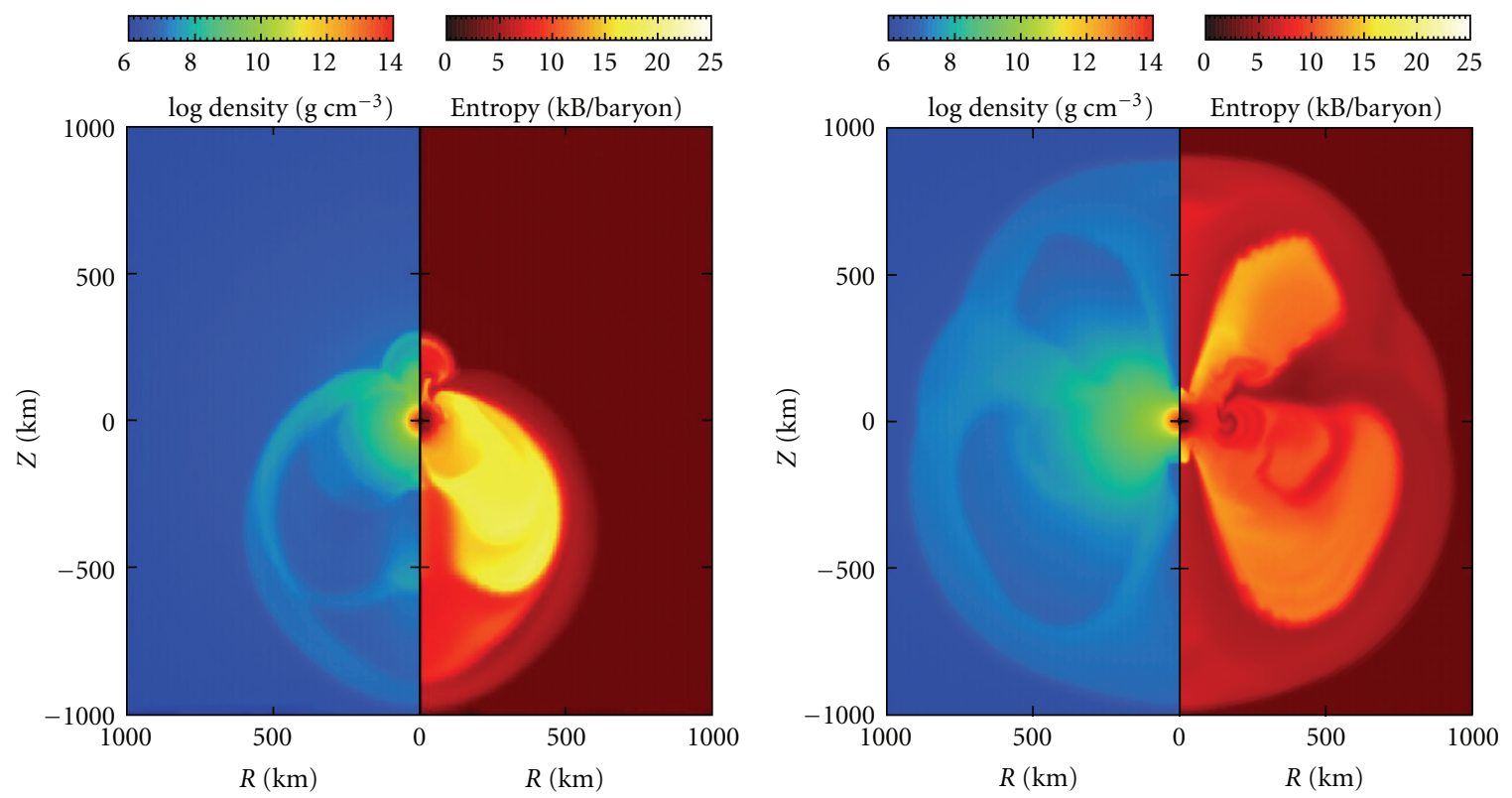

(b)

Figure 4: Time evolution of the explosion energy versus postbounce time for 2D models with and without rotation (a). The explosion energy is defined as the total energy (internal plus kinetic plus gravitational), integrated overall matter where the sum of the corresponding specific energies is positive. Models with "-hr" indicate the ones with higher numerical resolution, in which the mesh numbers in the lateral direction are doubled. (b) are snapshots of the density (left half) and the entropy (right half) for 1D (left) and 2D rotating (right) models at the epoch when the shock reaches $1000 \mathrm{~km}$. These figures are taken from [138].

diffusion in the 1D GR hydrodynamics (citing from his paper, "In this calculation, the neutrino luminosity of the core is found to be $10^{54} \mathrm{erg} / \mathrm{s}$, or $1 / 2$ a solar rest mass per second !! ... This is the mechanism which the supernova explodes." The neutrino luminosity rarely becomes so high in the modern simulations, but it is surprising that the potential impact of GR on the neutrino-heating mechanism was already indicated in the very first GR simulation). Using GR Boltzmann equations derived by Lindquist [148], Wilson [149] developed a 1D GR-radiation-hydrodynamic code including a more realistic (at the time) description of the collisional term than the one in [147]. By performing 1D GR hydrodynamic simulations that included a leakage scheme for neutrino cooling, hydrodynamical properties up to the prompt shock stagnation were studied in detail [150-152]. These pioneering studies, albeit using a much simplified neutrino physics than today, did provide a bottom-line of our current understanding of the supernova mechanism (see [153] for a complete list of references for the early GR studies). In the middle of the 1980s, Bruenn [134] developed a code that coupled 1D GR hydrodynamics to the MGFLD transport of order $(v / c)$ including the so-called standard set 


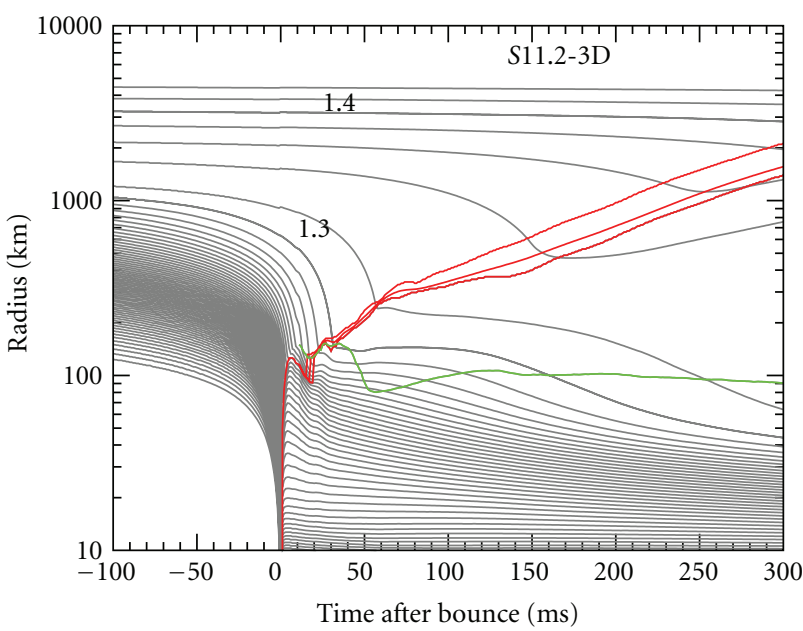

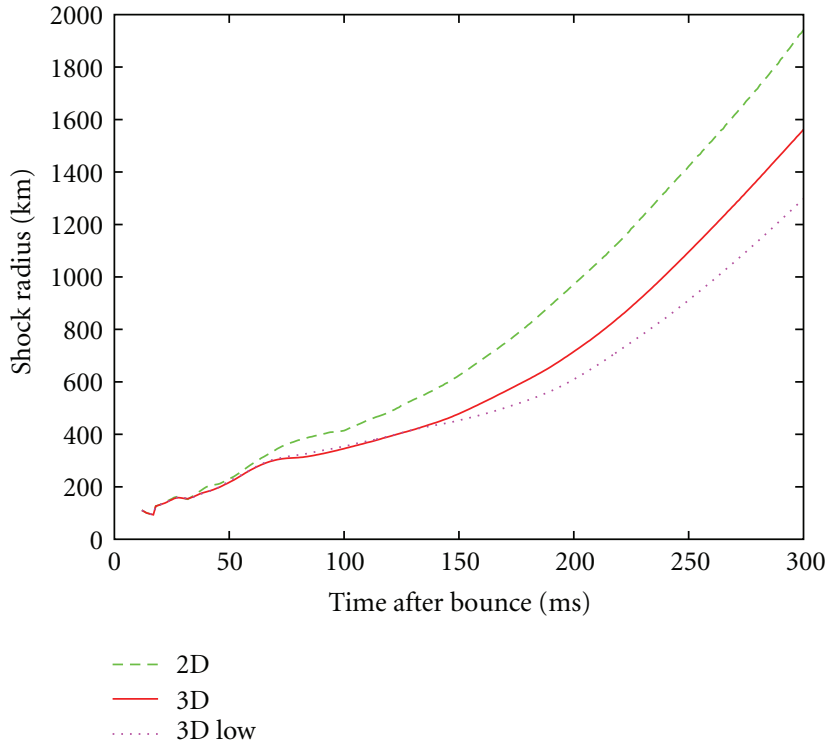

(b)

FIGURE 5: Time evolution of our 3D model [139] visualized by mass shell trajectories in thin gray lines (left panel). Thick red lines show the position of shock waves, noting that the maximum (top), average (middle), and the minimum (bottom) shock position are shown, respectively. The green line represents the shock position of the 1D model. "1.30" and "1.40" indicate the mass in unit of $M_{\odot}$ enclosed inside the mass-shell. Right panel shows the evolution of average shock radii for the 2D (green line) and 3D (red line) models. The "3D low" (pink line) corresponds to the low resolution 3D model, in which the mesh numbers are taken to be half of the standard model (see text). These figures are taken from [139].

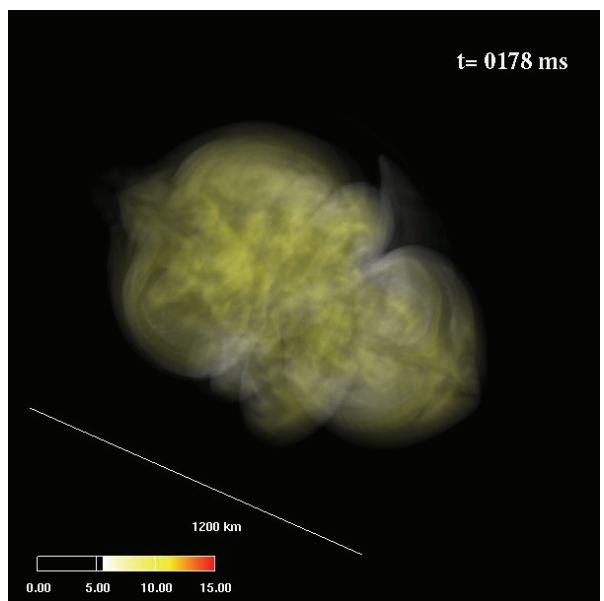

(a)

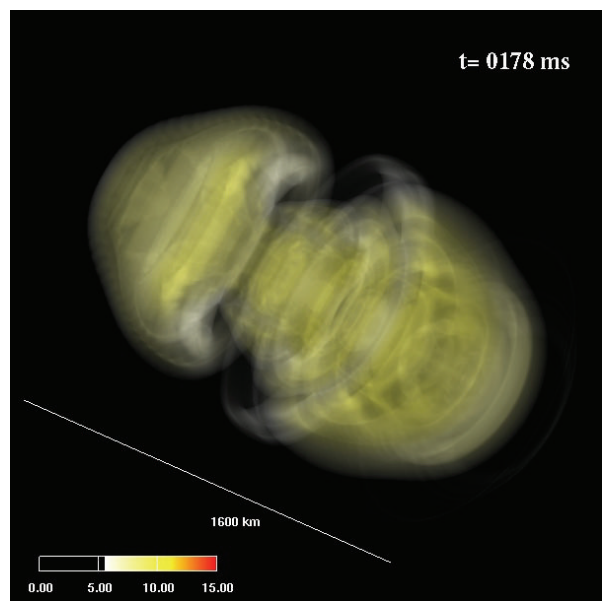

(b)

FiguRE 6: Volume rendering of entropy showing the blast morphology in our 3D (left) and 2D (right) model for the $11.2 M_{\odot}$ progenitor of Woosley et al. [118] (at $t=178 \mathrm{~ms}$ after bounce), respectively. The linear scale is indicated in each panel. These figures are taken from [139].

of neutrino interactions. Since the late 1990s, the ultimate 1D simulations, in which the GR Boltzmann transport is coupled to 1D GR hydrodynamics, have been made feasible by Yamada et al. [154-157] (very recently, they reported their success to develop the first multiangle, multienergy neutrino transport code in 3D [158]) and by Mezzacappa et al. [153, 159-161] (and by their collaborators).
Among them, Bruenn et al. [153] firstly showed that the neutrino luminosity and the average neutrino energy of any neutrino flavor during the shock reheating phase increase when switching from Newtonian to GR hydrodynamics. They also pointed out that the increase is larger in magnitude compared to the decrease due to redshift effects and gravitational time dilation. By employing the 


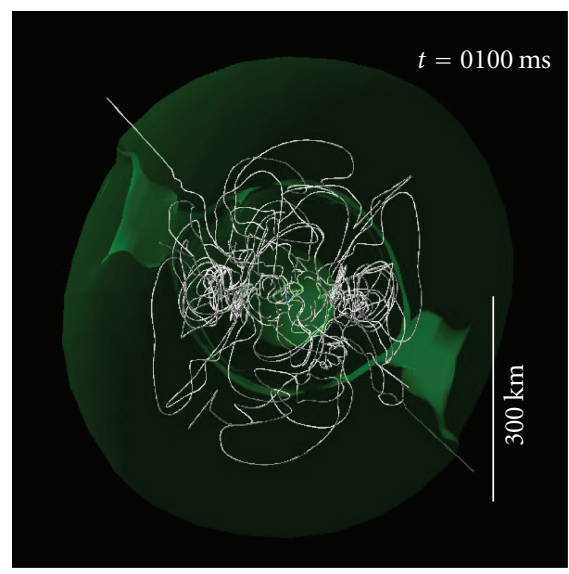

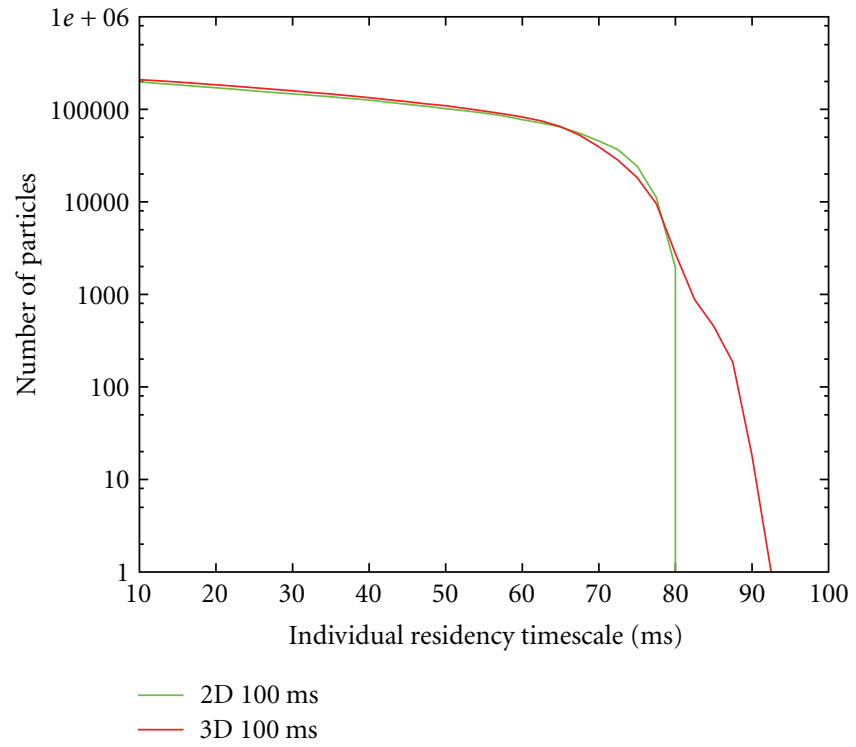

(b)

Figure 7: The left panel shows streamlines of selected tracer particles advecting through the shock wave to the PNS seen from the polar direction in our 3D model [139]. Several surfaces of constant entropy marking the position of the shock wave (greenish outside) and the PNS (indicated by the central sphere) are shown with the linear scale given in the right-bottom edge. The right panel shows the number of tracer-particles travelling in the gain region as a function of their individual residency time between the 2D and 3D model, at 100 ms after bounce. If the left panel were for 2D models, the streamlines would be seen as a superposition of circles with different diameters. The maximum residency timescale for the $3 \mathrm{D}$ model is shown to be longer than that in the corresponding $2 \mathrm{D}$ model. These figures are taken from [139].

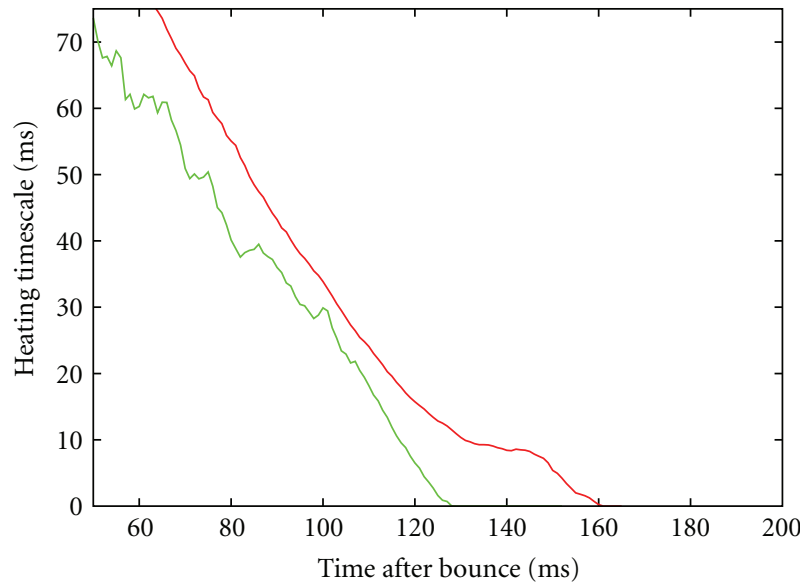

$-2 \mathrm{D}$
$-3 \mathrm{D}$

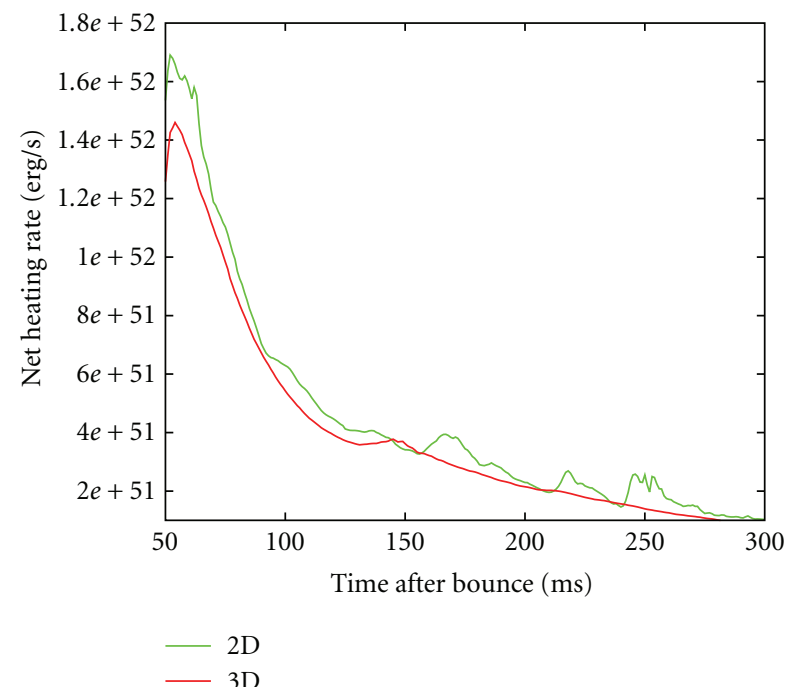

(b)

Figure 8: Time evolution of neutrino-heating timescale (left) and total net rate of neutrino heating (right) in our 2D (green line) and 3D models (red line). These figures are taken from [139].

current best available weak interactions, Lentz et al. [162] reported the update of Bruenn et al. [153] very recently. They showed that the omission of observer corrections in the transport equation particularly does harm to drive the neutrino-driven explosions. A disadvantageous trend of
GR to produce the neutrino-driven explosions has been commonly observed in these full-fledged 1D simulations; the residency time of material in the gain region becomes shorter due to the stronger gravitational pull. Due to this competition between the gain and loss effects in the end, 
GR works disadvantageously to facilitate the neutrino-driven explosions in 1D. In fact, the maximum shock extent in the postbounce phase is shown to be $20 \%$ smaller when switching from Newtonian to GR hydrodynamics (e.g., Figure 2 in [162]).

Among the most up-to-date multi-D models with spectral neutrino transport mentioned earlier (Table 1), the GR effects are best attempted to be modeled by using a modified gravitational potential that takes into account a 1D, post-Newtonian correction $[124,125,135,163]$. A possible drawback of this prescription is that a conservation law for the total energy cannot be guaranteed by adding an artificial term in the Poisson equation. Since the energy reservoir of the supernova engines is the gravitational binding energy, any potential inaccuracies in the argument of gravity should be avoided. There are a number of relativistic simulations of massive stellar collapse in full GR (e.g., 2D [164] or 3D $[165,166]$, and references therein) or using the conformallyflatness approximation (CFC) (e.g., $[130,131])$. Although extensive attempts have been made to include microphysics such as by the $Y_{e}$ formula [167] or by the neutrino leakage scheme [168], the effects of neutrino heating have yet to be included in them, which has been a main hindrance to study the GR effects on the multi-D neutrino-driven mechanism (see, however, $[129,169]$ ).

Putting things together, a complete "realistic" supernova model should naturally be done in full GR (MHD) with multi-D GR Boltzmann neutrino transport, in which a microphysical treatment of equation of state (EOS) and nuclear-neutrino interactions are implemented as realistically as possible. Unfortunately none of the currently published SN simulations satisfy the "ultimate" requirement (e.g., Table 1). In this sense, all the mentioned studies employ some approximations (with different levels of sophistication) towards the final goal.

In the same way, theoretical predictions of the $\mathrm{SN}$ multimessengers that one can obtain by analyzing the currently available numerical results, cannot unambiguously give us the final answer yet. Again we hereby note the feature of this paper which shows only a snapshot of the moving theoretical terrain. Keeping this caveat in mind, it is also true that a number of surprising GW features of CCSNe have been reported recently both by the firstprinciple simulations (e.g., in Table 1) and also by idealized simulations in which explosions are parametrically initiated mostly by the light-bulb scheme. As will be mentioned in the next section, the latter approach is also useful to get a better physical understanding of the GW signatures obtained in the first-principle simulations (in this sense, these two approaches are complimentary in understanding the GW signatures). Having shortly summarized a current status of CCSN simulations, we are now ready to move on to focus on the GW signatures from the next section.

2.2. Gravitational Waves. The paper by Müller [170] entitled as "Gravitational Radiation from Collapsing Rotating Stellar Cores" unquestionably opened our eyes to the importance of making the GW prediction based on realistic SN numerical modeling (see e.g., Section 2 in [23] for a summary of more earlier work which had mainly focused on the GW emission in very idealized systems such as in homogeneous spheroids and ellipsoids) (needless to say, this kind of approach is still very important to extract the physics of the GW emission mechanism). As one may expect from the title of his paper, rapid rotation, if it would exist in the precollapse iron core, leads to significant rotational flattening of the collapsing and bouncing core, which produces a timedependent quadrupole (or higher) GW emission. Following the first study by Müller, most studies of the past thirty have focused on the so-called bounce signals (e.g., [170-184]), and references therein).

As summarized by Ott [23], a number of important progresses have been recently made to understand features of the bounce signals by extensive 2D GR studies using the CFC approximation [180-182] and also by fully GR 3D simulations [177] both including realistic EOSs and a deleptonization effect based on 1D-Boltzmann simulations [185].

For the bounce signals having a strong and characteristic signature, the iron core must rotate enough rapidly. However recent stellar evolution calculations suggest that rapid rotation assumed in most of the previous studies is not canonical for progenitors with neutron star formations $[126,127,186]$. To explain the observed rotation periods of radio pulsars, the precollapse rotation periods are estimated to be larger than $\sim 100 \mathrm{sec}$ [186]. In such a slowly rotating case, the detection of the bounce signals becomes very hard even by next-generation laser interferometers for a Galactic supernova (e.g., [175]).

Besides the rapid rotation, convective matter motions and anisotropic neutrino emission in the postbounce phase are expected to be primary GW sources with comparable amplitudes to the bounce signals (e.g., [187] for a review). Thus far, various physical ingredients for producing asphericities and the resulting GWs in the postbounce phase have been studied, such as the roles of precollapse density inhomogeneities [102, 188, 189], moderate rotation of the iron core [190], nonaxisymmetric rotational instabilities [191, 192], g-modes [193] and r-modes pulsations [20] of PNSs, and the SASI [194-198] (see also [199] for the GW signals at the black hole formation). Among them, the most promising GW sources may be convection and SASI, because the degree of the initial inhomogeneities [200] and the growth of rotational instabilities as well as the r-modes and g-modes pulsations are rather uncertain.

Based on 2D simulations that parametrize the neutrino heating and cooling by the light-bulb scheme (which we shortly call as parametric SASI simulations hereafter), we pointed out that the GW amplitudes from anisotropic neutrino emission (as anisotropic matter motions generate GWs, anisotropic neutrino emission also gives rise to GWs, which has been originally pointed out in late 1970's by Epstein [201] and Turner and Wagoner [202] (see recent progress in [203]). It is expected as a primary GW source also in gamma-ray bursts [204-206] and Pop III stars [207]) increase almost monotonically with time, and that such signals may be visible to next-generation detectors for a 
Galactic source $[195,196]$. By performing such a parametric simulation but without the excision inside the PNS, Murphy et al. [198] showed that the GW signals from matter motions can be a good indicator of the explosion geometry. These features qualitatively agree with the ones obtained by Yakunin et al. [208] who reported exploding 2D simulations in which the ray-by-ray MGFLD neutrino transport is solved with the hydrodynamics (e.g., Oak-Ridge+ simulations in Table 1). Marek et al. [197] analyzed the GW emission based on their long-term 2D ray-by-ray Boltzmann simulations, which seem very close to produce explosions [125] (e.g., MPA simulations in Table 1). They also confirmed that the GWs from neutrinos with continuously growing amplitudes (but with the different sign of the amplitudes in [195, 196, 208]), are dominant over the ones from matter motions. They proposed that the third-generation class detectors such as the Einstein Telescope are required for detecting the GW signals with a good signal-to-noise ratio.

Regarding the GW predictions in 3D models, Müller and Janka [189] coined the first study to analyze the GW signature of 3D nonradial matter motion and anisotropic neutrino emission from prompt convection in the outer layers of a PNS during the first $30 \mathrm{~ms}$ after bounce. Their first 3D calculations using the light-bulb recipe were forced to be performed in a wedge of opening angle of $60^{\circ}$. Albeit with this limitation (probably coming from the computer power at that time), they obtained important findings that because of smaller convective activities inside the cone with slower overturn velocities, the GW amplitudes of their 3D models are more than a factor of 10 smaller than those of the corresponding 2D models, and the wave amplitudes from neutrinos are a factor of 10 larger than those due to nonradial matter motions. With another pioneering (2D) study by Burrows and Hayes [188] it is worth mentioning that those early studies had brought new blood into the conventional GW predictions, which illuminated the importance of the theoretical prediction of the neutrino GWs.

A series of findings obtained by Fryer et al. in early 2000s $[101,209]$ have illuminated also the importance of the 3D modeling. By running their 3D Newtonian SmoothedParticle-Hydrodynamic (SPH) code coupled to a gray fluxlimited neutrino transport scheme, they studied the GW emission due to the inhomogeneous core-collapse, core rotation, low-modes convection, and anisotropic neutrino emission. Although the early shock-revival and the subsequent powerful explosions obtained in these SPH simulations have yet to be confirmed by other groups, their approach paying particular attention to the multiple interplay between the explosion dynamics, the GW signatures, the kick and spins of pulsars, and also the nonspherical explosive nucleosynthesis, blazed a new path on which current supernova studies are progressing.

We also studied the GW signals from 3D models that mimic neutrino-driven explosions aided by the SASI [194, 196, 210]. In the series of our 3D experimental simulations, the light-bulb scheme was used to obtain explosions and the initial conditions were derived from a steady-state approximation of the postshock structure and the dynamics only outside an inner boundary at $50 \mathrm{~km}$ was solved. Based on the results, we show in the following that features of the gravitational waveforms obtained in $3 \mathrm{D}$ models are significantly different than those in $2 \mathrm{D}$, which tells us a necessity of 3D modeling for a reliable prediction of the GW signals.

\subsubsection{Stochastic Nature of Gravitational Waves in 3D Simu-} lations. Figure 9 shows the evolution of 3D hydrodynamic features from the onset of the nonlinear regime of SASI (top left) until the shock breakout (this corresponds to the shock emergence at the outer boundary of the computational domain ( $2000 \mathrm{~km}$ in radius)) (bottom right) with the gravitational waveform from neutrinos inserted in each panel. After about $100 \mathrm{~ms}$, the deformation of the standing shock becomes remarkable marking the epoch when the SASI enters the nonlinear regime (top left of Figure 9). At the same time, the gravitational amplitudes begin to deviate from zero. Comparing the top two panels in Figure 10, which shows the total amplitudes (top panel, neutrino + matter) and the neutrino contribution only (bottom), it can be seen that the overall structures of the waveforms are predominantly determined by the neutrino-originated GWs with the slower temporal variations ( $\gtrsim 30-50 \mathrm{~ms}$ ), to which the GWs from matter motions with rapid temporal variations ( $\lesssim 10 \mathrm{~ms}$ ) are superimposed.

As seen from the top right through bottom left to right panels of Figure 9, the major axis of the growth of SASI is shown to be not aligned with the symmetric axis $(z$-axis in the figure) and the flow inside the standing shock wave is not symmetric with respect to this major axis (see the first and third quadrants in Figure 9). This is a generic feature in the computed 3D models, which is in contrast to the axisymmetric case. The GW amplitudes from SASI in 2D showed an increasing trend with time due to the symmetry axis, along which SASI can develop preferentially $[195,196]$. Free from such a restriction, a variety of the waveforms is shown to appear (see waveforms inserted in Figure 5). Furthermore, the 3D standing shock can also oscillate in all directions, which leads to the smaller explosion anisotropy than 2D. With these two factors, the maximum amplitudes seen either from the equator or the pole become smaller than 2D. On the other hand, their sum in terms of the total radiated energy are found to be almost comparable between $2 \mathrm{D}$ and 3D models, which is likely to imply the energy equipartition with respect to the spatial dimensions.

The (two) pair panels of Figure 10 show the gravitational waveforms for models with different neutrino luminosity. The input luminosity for the pair panels (models A (top two) and B (bottom two)) differs only $0.5 \%$ (although any seed perturbation would demonstrate the chaotic behavior, we simply chose the value of $0.5 \%$ as a reference). Despite the slight difference in the luminosities, the waveforms of each polarization are shown to exhibit no systematic similarity when seen from the pole or equator. This also reflects a chaotic nature of the SASI influenced by small differences (see also [211]).

It should be noted that the approximations taken in the simulation, such as the excision inside the PNS with its 


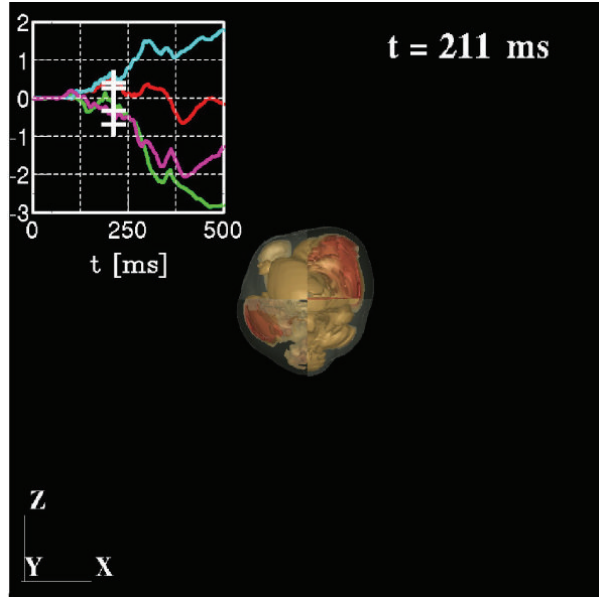

(a)

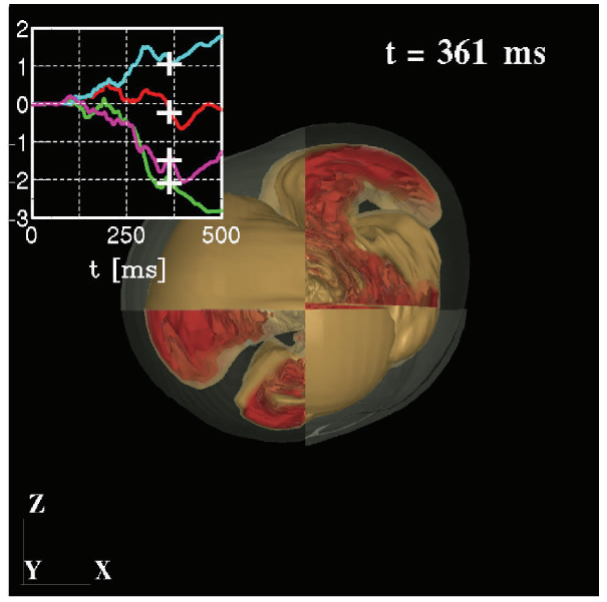

(c)

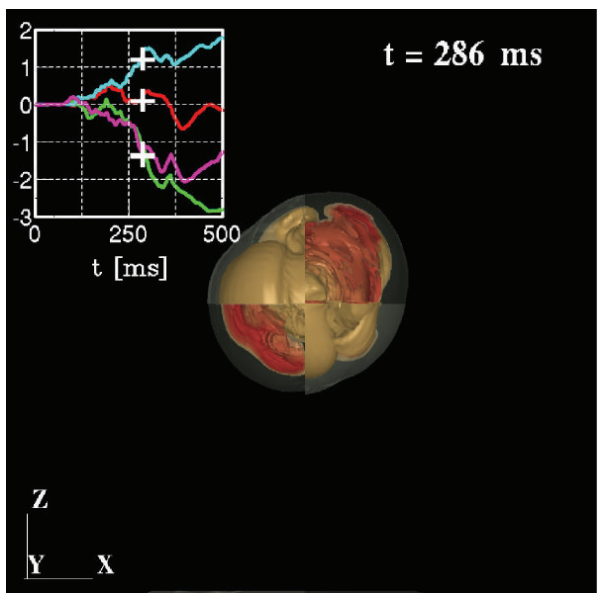

(b)

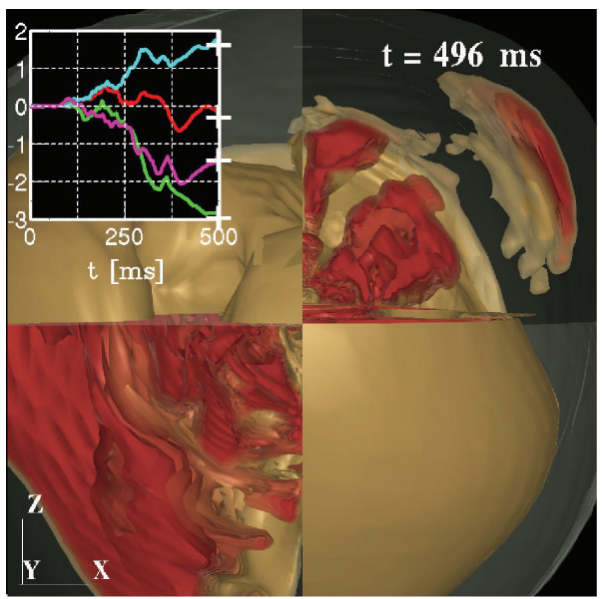

(d)

FIGURE 9: Four snapshots of the entropy distributions of a representative 3D supernova explosion model (corresponding to model A in [194]). The second and fourth quadrants of each panel show the surface of the standing shock wave. In the first and third quadrants, the profiles of the high entropy bubbles (colored by red) inside the section cut by the $Z X$ plane are shown. The side length of each plot is $1000 \mathrm{~km}$. The insets show the gravitational waveforms from anisotropic neutrino emissions, with "+" on each curve representing the time of the snapshot. Note that the colors of the curves are taken to be the same as the top panel of Figure 10. This figure is taken from [194].

fixed inner boundary and the light bulb approach with the isotropic luminosity constant with time, are the very first step to model the dynamics of the neutrino-heating explosion aided by SASI and study the resulting GWs. As already mentioned, the excision of the central regions inside PNSs may hinder the efficient gravitational emission of the oscillating neutron star [193] and the nonaxisymmetric instabilities $[184,192]$ of the PNSs, and the enhanced neutrino emissions inside the PNSs [197]. Bearing these caveats in mind, a piece of encouraging news is that the gravitational waveforms obtained in the 2D radiation-hydrodynamic simulations [208] are similar to the ones obtained in our 2D study using the light-bulb scheme [196]. More recently, Müller et al. [211] confirmed the stochastic nature by analyzing the GW features obtained in their 3D models [115]. The obtained GW amplitudes as well as the degree of anisotropic neutrino emission are almost comparable to our results, which are considerably smaller than 2D models [190, 197, 198, 208].

2.2.2. Breaking of the Stochasticity due to Stellar Rotation. More recently, we studied the effects of stellar rotation on the stochastic nature of the GWs mentioned above [210]. In 3D, the modes of SASI are divided into sloshing modes and spiral modes (e.g., [111]). Asymmetric $m=0$ modes so far studied in 2D models and axisymmetric $m \neq 0$ modes are classified into the sloshing modes, where $m$ stands for the azimuthal index of the spherical harmonics $Y_{l}^{m}$. In the latter situation, the $\pm m$ modes degenerate so that the $+m$ modes has the same amplitudes as the $-m$ modes. If random perturbations or uniformly rotating flows are imposed on these axisymmetric flows in the postbounce phase, the degeneracy is broken and 


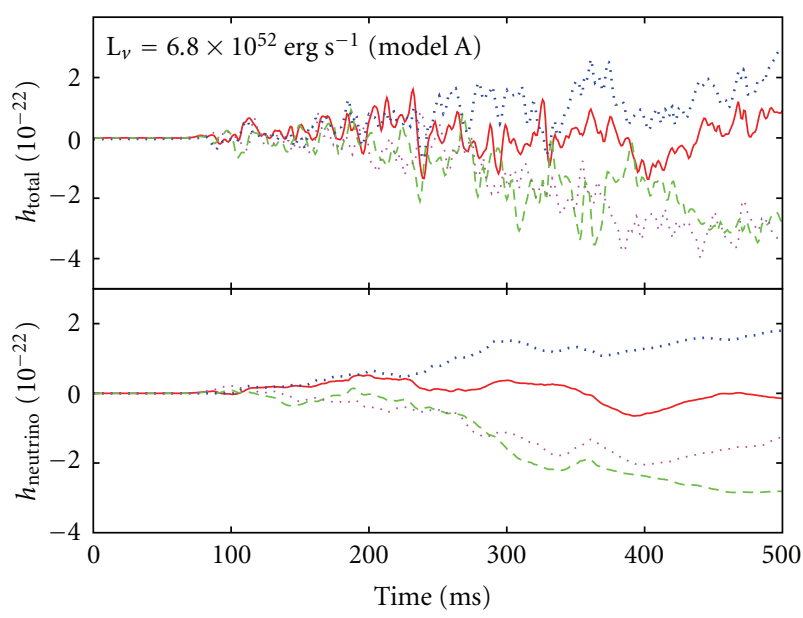

(a)

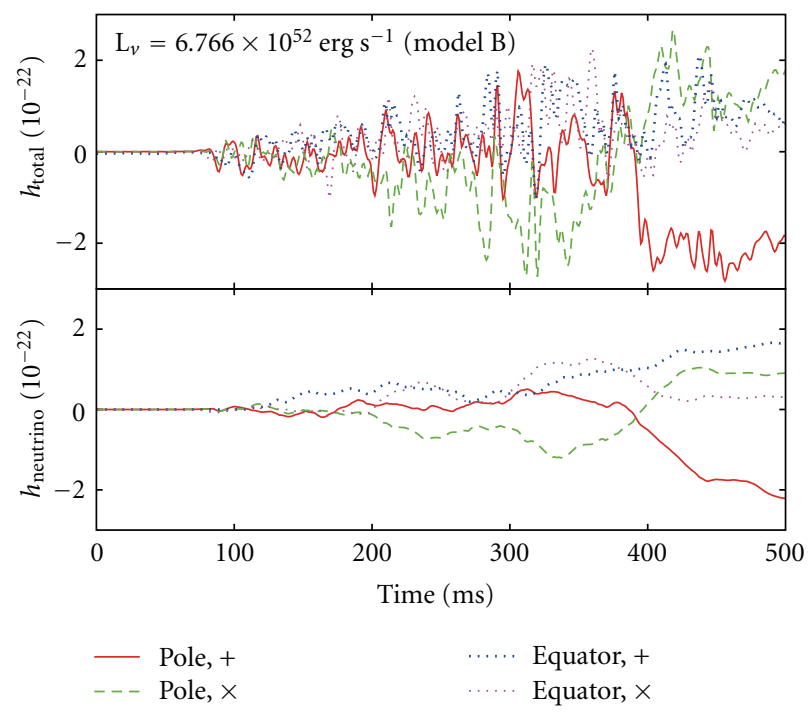

(b)

Figure 10: Gravitational waveforms from neutrinos (bottom) and from the sum of neutrinos and matter motions (top), seen from the polar axis and along the equator (indicated by "Pole" and "Equator") with polarization ( + or $\times$ modes) for two representative 3D models of A and B (see [194] for details). The distance to the SN is assumed to be $10 \mathrm{kpc}$.

the rotational modes emerge $[112,212]$. In this situation, the $+m$ modes has the different amplitudes from $-m$ modes. Such rotating nonaxisymmetric $m \neq 0$ modes are called as spiral modes. These nonaxisymmetric modes are expected to bring about a breakthrough in our supernova theory, because they can help to produce explosions more easily compared to $2 \mathrm{D}$ due to its extra degree of freedom [116], and also because they may have a potential to generate pulsar spins $([212,213]$, see also $[115,214])$. These findings illuminating the importance of stellar rotation motivated us to clarify how rotation that gives a special direction to the system (i.e., the spin axis), could affect the stochastic GW features which we observed in the absence of rotation (Section 2.2.1).
Figure 11 shows the gravitational waveform for a typical 3D model with rotation (left: total amplitudes, right: neutrino only). To construct a model with rotation, we give a uniform rotation on the flow advecting from the outer boundary of the iron core as in [112], whose specific angular momentum is assumed to agree with recent stellar evolution models [126, 127]. Comparing to Figure 10 (for models without rotation), one can clearly see a sudden rise in the GW amplitude after around $500 \mathrm{~ms}$ for the rotating model (blue line in Figure 11), which is the plus mode of the neutrino GW seen from the equator. By systematically changing the initial angular momentum and the input neutrino luminosities from the PNS, we computed fifteen 3D models in [210] and found that the GW features mentioned above were common among the models.

Figure 12 illustrates a typical snapshot of the flow fields for the rotating model (corresponding to the one in Figure 11) when the spiral SASI modes have already entered the nonlinear regime, seen from the pole (right panel) or from the equator (left panel), respectively. From the left panel, one may guess the presence of the sloshing modes that happen to develop along the rotational axis ( $Z$-axis) at this epoch. It should be emphasized that although the dominance of $h_{\nu,+}^{\text {equ }}$ observed in the current 3D simulations is similar to the one obtained in previous 2D studies [196], its origin has nothing to do with the coordinate symmetry axis. The preferred direction here is determined by the spin axis. Free from the 2D axis effects, the major axis of the sloshing SASI mode changes stochastically with time, and the flow patterns behind the standing shock simultaneously change in every direction like the nonrotating models. As a result, the sloshing modes can make only a small contribution to the GW emission. The remaining possibility is that the spiral flows seen in the right panel should be a key importance to understand the GW feature mentioned above. In fact, by analyzing the matter distribution on the equatorial plane, we find that the compression of matter is more enhanced in the vicinity of the equatorial plane due to the growth of the spiral SASI modes, leading to the formation of the spiral flows circulating around the spin axis with higher temperatures. As a result, the neutrino emission seen parallel to the spin axis becomes higher than the ones seen from the other direction. Remembering that the lateral-angle $(\theta)$ dependent function of the GW formulae (e.g., in (9) in [196]) is positive near the north and south polar caps, the dominance of the polar neutrino luminosities leads to make the positively growing feature of $h_{v,+}^{\text {equ }}$ in Figure 11 (blue line). From the spectral analysis of the gravitational waveform (Figure 13), it can be readily seen that it is not easy to detect these neutrinooriginated GW signatures with slower temporal evolution ( $\mathrm{O}(10) \mathrm{ms}$ ) by ground-based detectors whose sensitivity is limited mainly by the seismic noises at such lower frequencies. However these signals may be detectable by the recently proposed future space interferometers like Fabry-Perot type DECIGO ([217], black line in Figure 13). Contributed by the neutrino GWs in the lower frequency domains, the total GW spectrum tends to become rather flat over a broad frequency range below $\sim 100 \mathrm{~Hz}$. These GW features obtained in the context of the SASI-aided neutrino-driven mechanism 


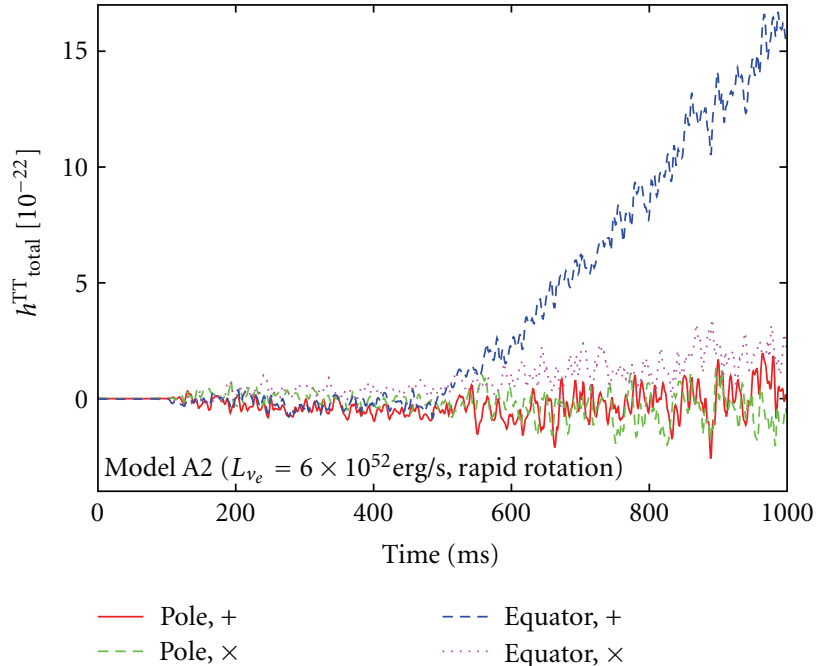

(a)

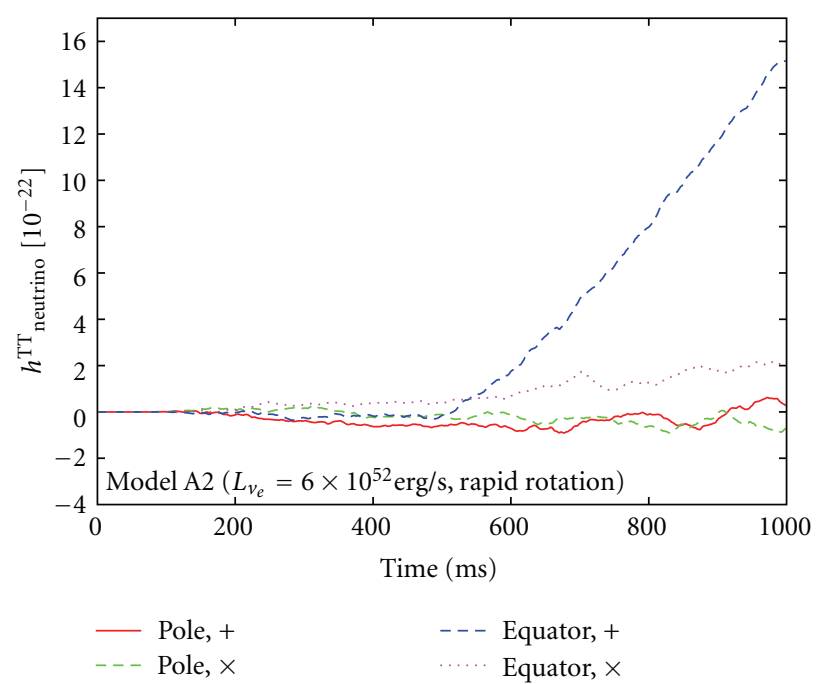

(b)

FIGURE 11: Gravitational waveforms from the sum of neutrinos and matter motions (left) and only from neutrinos (right) for a 3D model with rotation (from [210]). The time is measured from the epoch when the neutrino luminosity is injected from the surface of the neutrino sphere. For this $3 \mathrm{D}$ model with rotation, the rotational flow is imposed to advect to the PNS surface at around $t=400 \mathrm{~ms}$. The supernova is assumed to be located at the distance of $10 \mathrm{kpc}$.

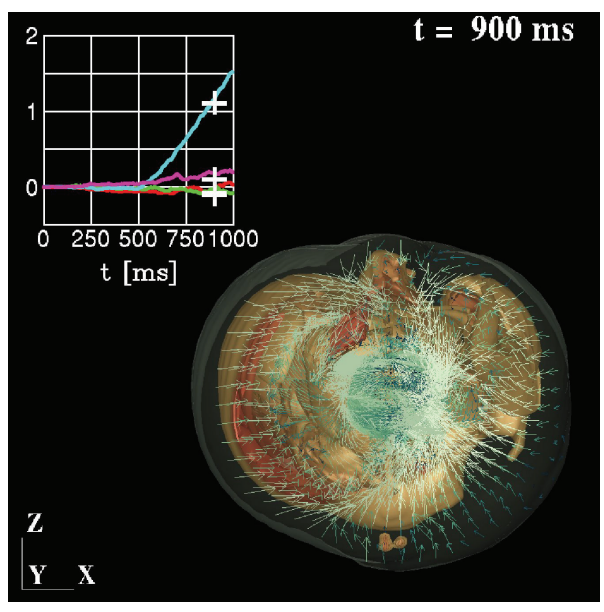

(a)

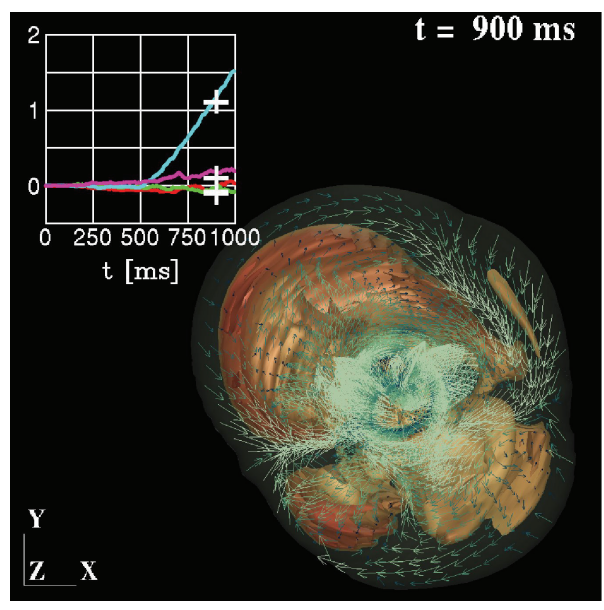

(b)

FIGURE 12: Partial cutaway of the entropy isosurfaces and the velocity vectors on the cutting plane for a 3D model that includes rotation. Left and right panels are for the equatorial and polar observer, respectively. The insets show the gravitational waveforms with " + " on each curves representing the time of the snapshot. Note that the colors of the curves are taken to be the same as the top panel of Figure 10. This figure is taken from [210].

are different from the ones expected in the other candidate mechanisms, such as the MHD mechanism (e.g., [219]) and the acoustic mechanism [193]. Therefore the detection of such signals could be expected to provide an important probe into the explosion mechanism (e.g., [23, 220]).

We like to draw a caution that most of the 3D models cut out the PNS and the neutrino transport is approximated by a simple light-bulb scheme [210] or by the gray transport scheme [211]. Needless to say, these exploratory approaches are but the very first step to model the neutrino-heating explosion and to study the resulting GWs. As already mentioned, the excision of the central regions inside PNSs truncates the feedback between the mass accretion to the PNS and the resulting neutrino luminosity, which should affect the features of the neutrino GWs. By the cutout, efficient GW emission of the oscillating neutron star [193] and nonaxisymmetric instabilities $[183,184]$ of the PNSs, and the enhanced neutrino emissions inside the PNSs [197] cannot be treated in principle. To elucidate the GW signatures in a more quantitative manner, full 3D simulations with spectral 


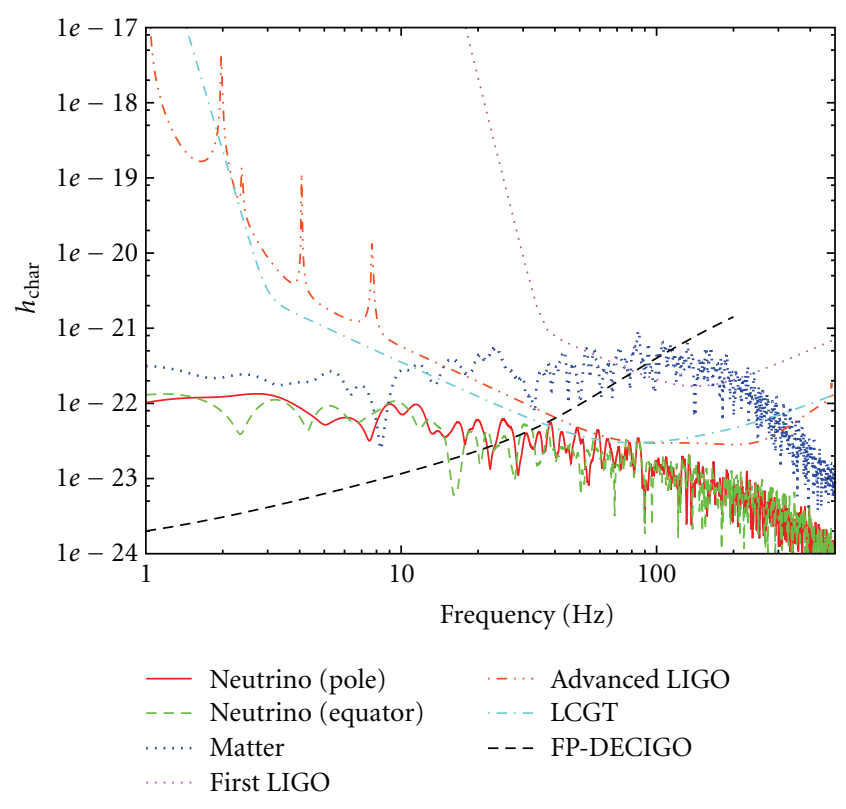

FIGURE 13: Spectral distributions of GWs from matter motions ("Matter") and neutrino emission ("Neutrino") seen from the pole or the equator for a representative 3D rotating model (e.g., [210]) with the expected detection limits of TAMA300 [12], first LIGO and advanced LIGO [215], Large-scale Cryogenic Gravitational wave Telescope (LCGT) [216] and Fabry-Perot type DECIGO [217]. It is noted that $h_{\text {char }}$ is the characteristic gravitational wave strain defined in [218]. The distance to the supernova is assumed to be $10 \mathrm{kpc}$. Note that for the matter signal, the + mode seen from the polar direction is plotted (from [210]).

neutrino transport are apparently needed (e.g., Section 2.1). This is unquestionably a vast virgin territory awaited to be explored for the future.

\subsection{Explosive Nucleosynthesis. In this section, we proceed} to discuss possible signatures of supernova nucleosynthesis. The study of nucleosynthesis is of primary importance to unveil the origins of heavy elements. It could also provide a valuable information of the ejecta morphology by observing the aspherical distributions of the synthesized elements especially for a nearby CCSN event (note that nucleosynthesis is not critical for the modeling of the light-curve and spectra for the most frequent types of SNe II-P). In the following, we first present a short overview paying particular attention to explosive nucleosynthesis, and then discuss possible observational signatures that would imprint information of multidimensionalities of the supernova engine.

When in a successful explosion the shock passes through the outer shells, its high temperature induces an explosive nucleosynthesis on short timescales (e.g., [118, 119, 221], and collective references in [222]). The observational determination of the masses of the three main radioactive isotopes ${ }^{56} \mathrm{Ni},{ }^{57} \mathrm{Ni}$, and ${ }^{44} \mathrm{Ti}$ sets one of the main constraints on the explosion dynamics, because the production of these elements is sensitive to the track of density and temperature that the expanding material traces (e.g., [223]). During the shock propagation, iron group elements such as ${ }^{56} \mathrm{Ni}$ and its daughter nucleus ${ }^{56} \mathrm{Co}$ are predominantly produced, which are radioactive with a lifetime of 8.8 days and 111.5 days, respectively. Most CCSNe enter the so-called nebular phase after the first few months when the expanding ejecta becomes optically thin in the continuum. In the early nebular phase, ${ }^{56} \mathrm{Co}$ is the major nuclear power source. As long as the decay particles are trapped by the ejecta, the radiation energy supplied by radioactivity is emitted instantaneously, so that the light curve can be described by an exponential decay with time, simply tracing the decay of the ${ }^{56} \mathrm{Co}$ nuclide. To explain the bolometric light curve of SN1987A in such a phase, the ${ }^{56} \mathrm{Ni}$ mass was determined to be $0.07 M_{\odot}[224]$.

After several years of explosion, the radioactive output from the ejecta no longer balances with the instantaneous input by radioactivity, because the reprocessing timescale is going to be longer [225]. The bolometric light curve is affected by the delayed release of the ionization energy. After that, a self-consistent modeling is needed, in which one should include a detailed calculation of the gammaray/positron thermalization and a determination of the timedependent temperature, ionization, and excitation (e.g., [225-228] and references therein). Such a time-dependent modeling by Fransson and Kozma [225] revealed the ${ }^{57} \mathrm{Ni}$ mass of $\sim 3.3 \times 10^{-3} M_{\odot}$ of SN1987A, which agrees well with observations (e.g., $[229,230]$ and collective references in [231]).

By the similar reason to ${ }^{57} \mathrm{Ni}$ just mentioned above, the determination of the ${ }^{44} \mathrm{Ti}$ is also complicated. The most recent study by Jerkstrand et al. [228] gives an estimate of the ${ }^{44} \mathrm{Ti}$ to be $1.5_{-0.5}^{+0.5} \times 10^{-4} M_{\odot}$, which is in good agreement with the eight-year spectrum analysis of SN1987A (e.g., [232], see also [233]). As shown above, the amount of ${ }^{44} \mathrm{Ti}$ is typically one order-of-magnitude smaller than that of ${ }^{57} \mathrm{Ni}$, however it is crucially important for young supernova remnants due to its long lifetime ( $~ 86$ years). It is worth mentioning that NASA will launch the satellite NuSTAR (Nuclear Spectroscopic Telescope Array) to study ${ }^{44} \mathrm{Ti}$ production in CCSNe. The detector will be able to map out the ${ }^{44} \mathrm{Ti}$ distribution of the supernova remnant Cassiopeia $\mathrm{A}$ and can get velocity distributions of the ${ }^{44} \mathrm{Ti}$ in SN 1987A. By comparing detailed modeling of the SN nucleosynthesis in the context of $2 \mathrm{D}$ and $3 \mathrm{D}$ models (e.g., $[234,235])$, these are expected to provide both direct probes of the explosion asymmetry.

Ever since SN1987A, challenges to the classical spherical modeling $[119,221,236,237]$ have been built also in the SN nucleosynthesis (likewise in the explosion theory and GWs mentioned so far). For many years it has been customary to simulate explosions and the effects of the shock wave on the explosive nucleosynthesis by igniting a thermal bomb in the star's interior or by initiating the explosion by a strong push with a piston. $2 \mathrm{D}$ simulations with manually imparted asymmetries showed that bipolar explosion scenarios could account for enhanced ${ }^{44} \mathrm{Ti}$ synthesis along the poles as indicated in SN1987A (e.g., [35]). More recently, 3D effects have been more elaborately studied ([238], see also [239]) as well as the impacts of different explosions by employing a number of progenitors [240] or by assuming a jet-like 
explosion [241, 242], which is one of the possible candidates of hypernovae (e.g., [243]).

In addition to the above-mentioned work, nucleosynthesis in a more realistic simulation that model multi-D neutrino-driven explosions has been also extensively studied (e.g., [33, 43, 244] and references therein). Although a small network has ever been included in the computations, these 2D simulations employing a light-bulb scheme [33] or a more accurate gray transport scheme $[43,44]$ have made it possible to elucidate nucleosynthesis inside from the iron core after the shock-revival up to explosion in a more consistent manner. Kifonidis et al. [43] demonstrated that the SASI-aided low-mode explosions can naturally explain masses and distributions of the synthesized elements observed in SN1987A. Their recent 3D results by Hammer et al. [34] show that the 3D effects change the velocity profiles as well as the growth of the Rayleigh-Taylor instability, which affects properties of the SN ejecta. In simulations with spectral neutrino transport, a new nucleosynthesis process, the so-called $\nu$ p process, is reported to successfully explain some light proton-rich ( $p$-)nuclei including ${ }^{92,94} \mathrm{Mo}$ and ${ }^{96,98} \mathrm{Ru}$ (e.g., [245-247] and references therein). It should be noted that self-consistent simulations are currently too computationally expensive to follow the dynamics of the expanding shock far outside the central iron core $(\gg 1 \mathrm{~s}$ after bounce) where explosive nucleosynthesis takes place. On the other hand, the light-bulb scheme is not accurate to determine a sensitive balance between neutrino captures proceeding via $v_{e}$ or $\bar{v}_{e}$, which is decisive for quantifying the $\nu \mathrm{p}$ processes. Therefore the two approaches, namely, lightbulb scheme versus self-consistent neutrino transport, may be regarded as playing a complimentary role at present.

In the next section, we briefly summarize our findings on explosive nucleosynthesis in our 2D models that utilize the light-bulb scheme to trigger explosions (e.g., [248] for more details). By changing neutrino luminosities from PNSs systematically $\left(L_{\nu}\right)$, we discuss how the multidimensionality formed by the SASI and convection could impact on the explosive nucleosynthesis. We employ a nonrotating $15 M_{\odot}$ star with solar metalicity, which has been a beststudied supernova progenitor. The abundance pattern of the synthesized elements is estimated by a post-processing procedure, in which we have adopted a large nuclear network continuously maintained by the Joint Institute for Nuclear Astrophysics (JINA) REACLIB project [249]. Our readers might wonder why we are returning to $2 \mathrm{D}$ results here again after referring to 3D studies in Section 2.2. This is simply because our $3 \mathrm{D}$ project for the nucleosynthesis is currently in progress, and we like to note again the feature of this paper which shows only a snapshot of the moving theoretical terrain.

\subsubsection{Symmetry Breaking in Explosive Nucleosynthesis.} Figure 14 shows how explosion energies (left panel), explosion timescales, as well as the PNS masses (right panel) change with the input neutrino luminosity $\left(L_{v_{e}}\right)$ for the $2 \mathrm{D}$ parametric explosion models (e.g., [248] for more details). As seen, higher $L_{\nu_{e}}$ makes explosion energy greater, the onset of explosion $\left(t_{\exp }\right)$ earlier, the PNS masses ( $\left.M_{\mathrm{PNS}}\right)$ smaller. The PNS masses range in (1.54-1.62) $M_{\odot}$ for models with higher luminosity $\left(L_{v_{e}} \gtrsim 4.25 \times 10^{52} \mathrm{erg} \mathrm{s}^{-1}\right.$, panel (b), blue line), which are comparable to the baryonic mass (around $1.5 M_{\odot}$ ) of neutron stars observed in binaries [250]. The explosion energies for these high luminosity models (panel (a), red line) are also close to the canonical supernova kinetic energy of $10^{51} \mathrm{erg}$. To discuss explosive nucleosynthesis in the following, we thus choose to focus on the model with $L_{\nu_{e}}=4.5 \times 10^{52} \mathrm{erg} \mathrm{s}^{-1}$ as a reference.

For the model, Figure 15 shows a snapshot of internal energy (left panel) and abundances of ejecta $(\mathrm{O}, \mathrm{Si}, \mathrm{Fe})$ when the SASI-aided low-mode shock is propagating in the O-rich layers at around $\sim 20000 \mathrm{~km}$ along the pole. Ejecta with higher internal energies (left panel, colored by red) concentrate on the shock front particularly in polar regions $(\theta<\pi / 4, \theta>3 \pi / 4)$, where both $\mathrm{Si}$ and $\mathrm{O}$ burning proceed to produce abundantly ${ }^{56} \mathrm{Ni}$ and ${ }^{28} \mathrm{Si}$. These aspherical element distributions might be responsible for anisotropies of the SN ejecta as observed in SN1987A. More recently, Kimura et al. [251, 252] have analyzed the metal distribution of the Cygnus loop and pointed out that the progenitor of the Cygnus loop is a CCSN explosion whose progenitor mass ranges in $\sim 12-15 M_{\odot}$. Since the material in this middle-aged supernova remnant has not been completely mixed yet, the observed asymmetry is considered to still remain a trace of inhomogeneity produced at the moment of explosion. The asymmetries of the $\mathrm{SN}$ ejecta obtained in our 2D explosion model of a $15 M_{\odot}$ progenitor have a close correlation with the ones in the Cygnus loop (see [248] for more detailed comparison). This might allow us to speculate that globally asymmetric explosions induced by the SASI-aided neutrinoheating mechanism could account for the origin.

Concerning the synthesized amount of ${ }^{56} \mathrm{Ni}$ and ${ }^{44} \mathrm{Ti}$, they are $\sim 0.06 M_{\odot}$ and $4 \times 10^{-5} M_{\odot}$, respectively, for the model (bottom panel in Figure 16). For SN1987A, masses of ${ }^{56} \mathrm{Ni}$ and ${ }^{44} \mathrm{Ti}$ are deduced to be $\sim 0.07 M_{\odot}[27,253]$ and $1-2 \times 10^{-4} M_{\odot}$ ([36], and references therein). The obtained mass of ${ }^{44} \mathrm{Ti}$ is, therefore, not enough for SN1987A as well as for Cas A $\left(1.6_{-0.3}^{+0.6} \times 10^{-4} M_{\odot}\right)[254]$. The shortage of ${ }^{44} \mathrm{Ti}$ is a long-standing problem, which might be solved by $3 \mathrm{D}$ effects [34]. For the abundance patterns, our reference model (top and middle panels in Figure 16) is shown to reproduce a similar trend to the solar system (top panel, Figure 16), which is in sharp contrast to the model with lower neutrino luminosity (middle panel, Figure 16).

Finally, we note that there should be at least two barriers to get over in the current-generation studies of the CCSN nucleosynthesis. As mentioned already, the first one is the need of radiation hydrodynamic simulations that follow the dynamics from gravitational collapse, shock-revival, shock propagation in the stellar mantle, to stellar explosions in a self-consistent manner. The second one is the need of computing light curves, spectra, and the degree of polarizations in $2 \mathrm{D}$ or $3 \mathrm{D}$ hydrodynamic models, for which one needs to solve the multi-D photon transport coupled with multiD hydrodynamics. Unfortunately however, the marriage of the two items, albeit ultimately needed for clarifying the photon messengers, may not be done immediately due to 


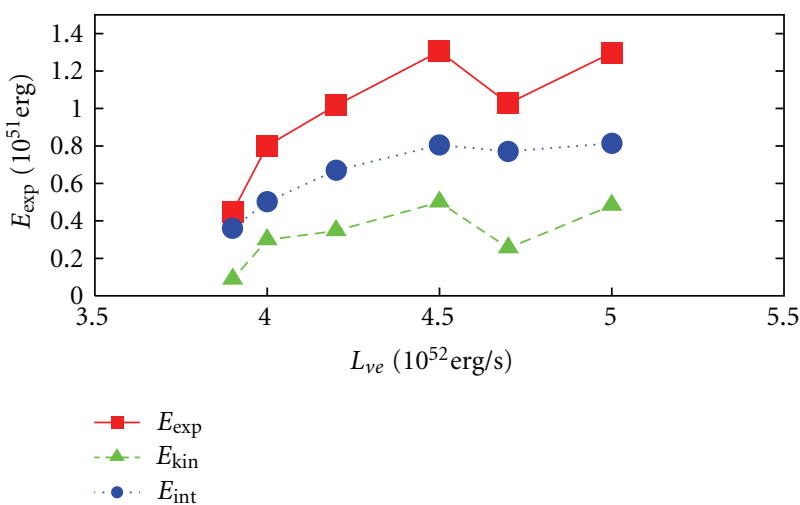

(a)

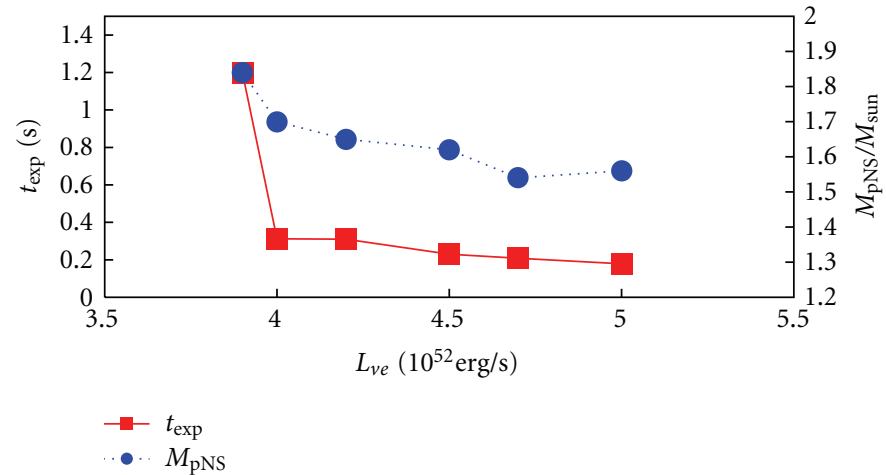

(b)

Figure 14: Panel (a) shows explosion energies (red line) versus input neutrino luminosities $\left(L_{v_{e}}\right)$, where green and blue lines show contributions from kinetic and thermal energy, respectively. Panel (b) shows the mass of protoneutron star ( $\left.M_{\mathrm{PNS}}\right)$ and the explosion timescales $\left(t_{\exp }\right)$ as a function of $L_{\gamma_{e}} . M_{\mathrm{PNS}}$ is estimated as the enclosed mass exceeding $10^{12} \mathrm{~g} \mathrm{~cm}^{-3}$ and $t_{\exp }$ as the time scale when the mass ejection rate at $100 \mathrm{~km}$ drops down to $0.1 M_{\odot} / \mathrm{s}$ in our $2 \mathrm{D}$ simulations (from [248]).

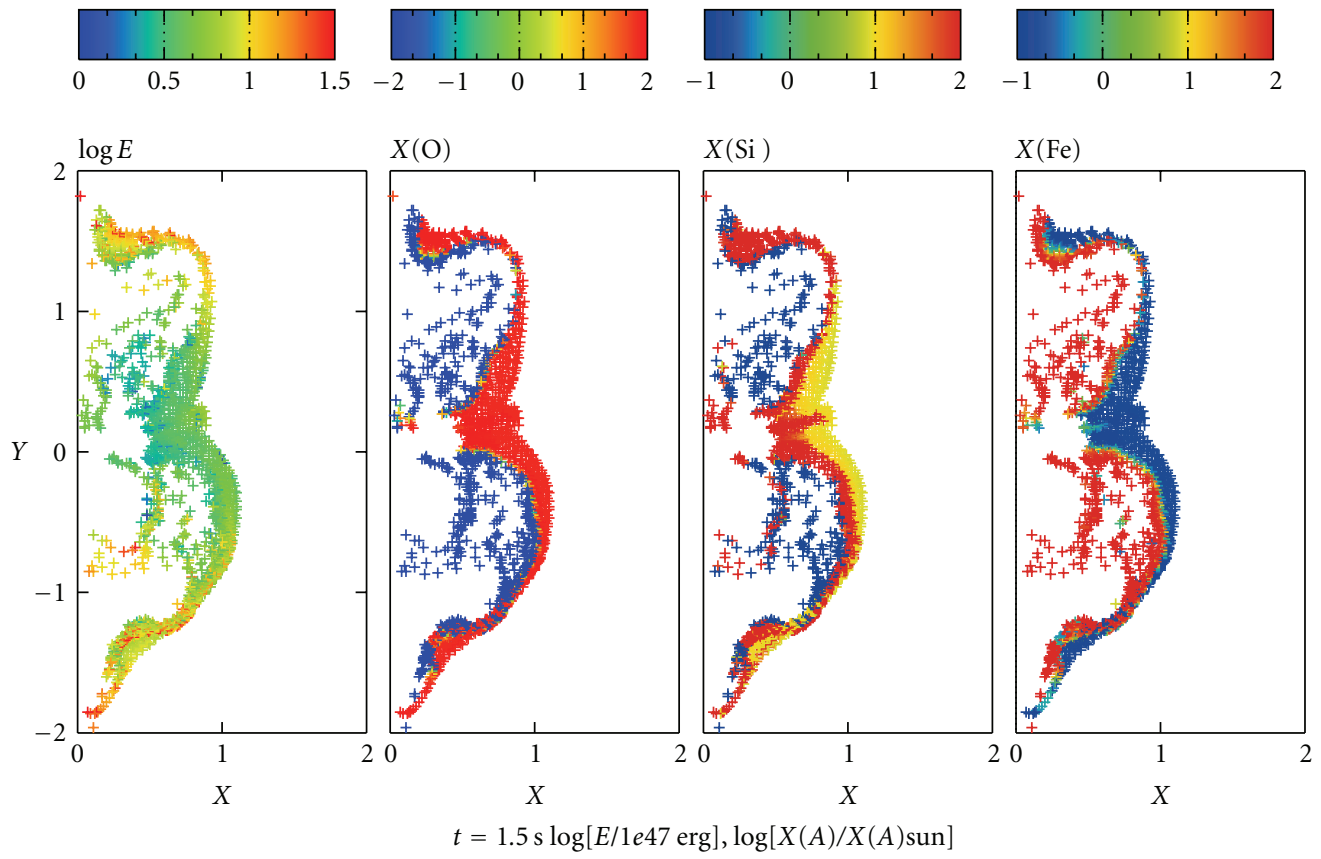

FIGURE 15: Distribution of internal energy (left panel) and abundances of the ejecta ( $\mathrm{O}, \mathrm{Si}$, Fe from left to right, normalized to solar) when the nonspherical shock is propagating in the O-rich layers at around $\sim 20000 \mathrm{~km}$ along the pole $(\sim 1.5 \mathrm{~s}$ after bounce). For this model, the input neutrino luminosity is set as $L_{\gamma_{e}}=4.5 \times 10^{52} \mathrm{erg} \mathrm{s}^{-1}$. Note that Fe in the right panel is the sum of ${ }^{56} \mathrm{Fe}$, ${ }^{56} \mathrm{Ni}$, and ${ }^{54} \mathrm{Fe}$ (from $[248]$ ).

their computational expensiveness. For example, one needs to follow the dynamics more than $\sim 1$ day after the onset of gravitational core-collapse for computing the explosive nucleosynthesis, because the envelop of a typical supernova progenitor extends up to a radius of $\sim 10^{12} \mathrm{~cm}$. It takes furthermore more than $\sim 1$ week before the shock propagation enters to the so-called homologous phase. At present, the best available numerical simulation to this end is limited to $2 \mathrm{D}$ (e.g., [244]). For discussing anisotropies in emission-line profiles, one needs to follow the dynamics later than $\sim 1$ year after explosions when the remnant becomes transparent. It is a very challenging (but very important) task for the first principle simulations to overcome the big gaps regarding the very different timescales. An encouraging news is that several groups are pursuing it with the use of advanced numerical techniques such as the adaptive-mesh-refinement approach [116] or Ying-Yang gridding [115]. With an accelerating power (like peta- or exa-scale) of supercomputers, it would be unsurprising that the next (or the next after next!) generation supernova modellers can execute the ultimate 


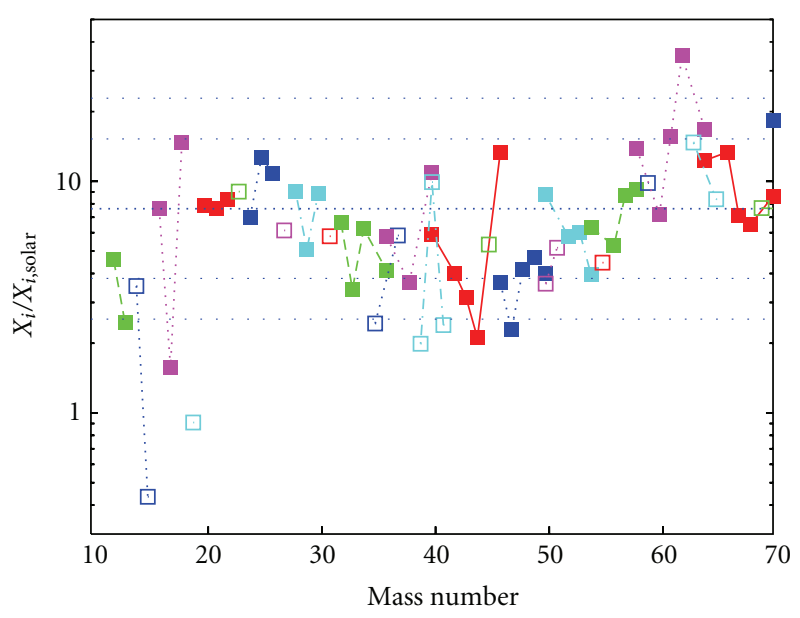

(a)

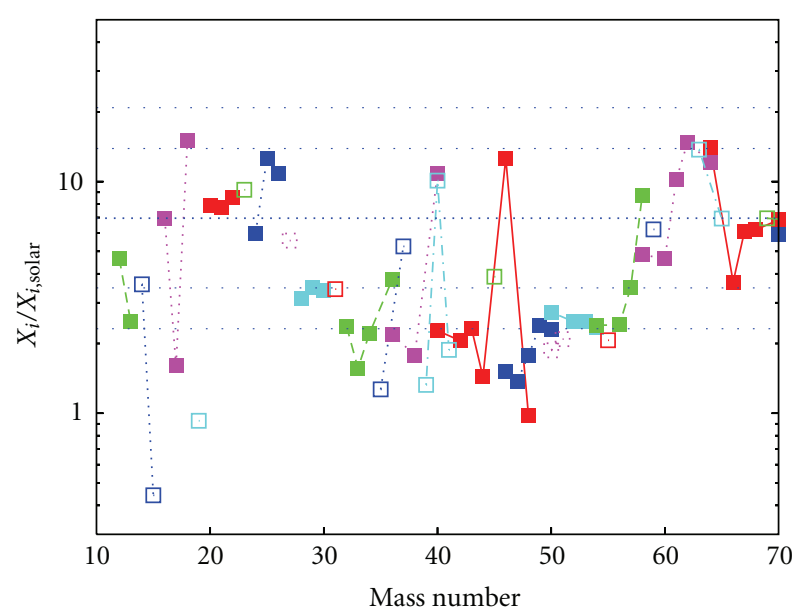

(b)

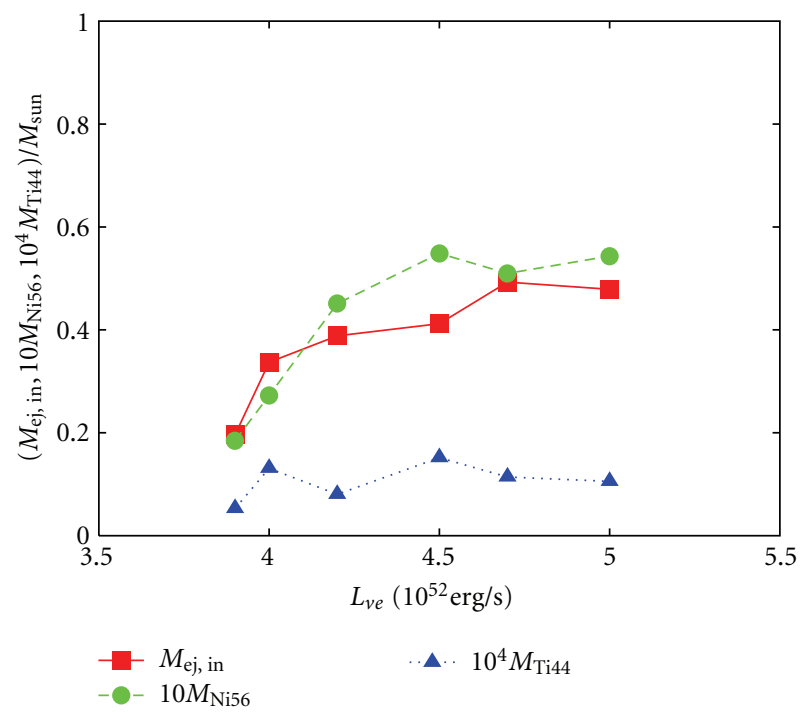

(c)

Figure 16: Bottom panel shows masses of ejecta $\left(M_{\mathrm{ej}, \text { in }}\right)$ and masses of radioactives $\left({ }^{56} \mathrm{Ni}\right.$ and $\left.{ }^{44} \mathrm{Ti}\right)$ as a function of the input neutrino luminosity. Top and middle panels show element abundance yields $\left(X_{i} / X_{\odot, i}\right.$, normalized to solar) versus mass number for our reference model of $L_{\gamma_{e}}=4.5 \times 10^{52} \mathrm{erg} \mathrm{s}^{-1}$ and for the middle panel $L_{\gamma_{e}}=3.9 \times 10^{52} \mathrm{erg} \mathrm{s}^{-1}$, respectively. Thick horizontal-dashed lines represent a factor equals to that of ${ }^{16} \mathrm{O}$, while two normal and two thin lines denote a factor equals to that of ${ }^{16} \mathrm{O}$ times $2,1 / 2,3$, and $1 / 3$, respectively. These figures are taken from [248].

ab initio simulations, whose findings would be immediately used to make a more precise prediction of the photon messengers.

\section{MHD Mechanism}

In this section, we move on to focus on the MHD mechanism. After we briefly summarize the current status of this topic below, we mention the explosion dynamics that proceeds by the field-wrapping mechanism in Section 3(a) and discuss properties of the so-called magnetorotational instability (MRI) in Sections 3(b) and 3(c) based on our preliminary results. Possible signatures of GWs and neutrinos are discussed in Sections 3.1 and 3.2, respectively.

Numerical simulations of MHD stellar explosions have started already in early 1970s shortly after the discovery of pulsars [255-258]. However, it is rather only recently that the MHD studies come back to the front-end topics in the supernova research followed by a number of extensive MHD simulations (e.g., [46, 175, 207, 259-270] for references therein). Main reasons for this activity are observations indicating very asymmetric explosions $[38,40]$, and the interpretation of magnetars [271, 272], collapsars and their 
relevance to gamma-ray bursts [273-275] as a possible outcome of the magnetorotational core-collapse of massive stars.

The MHD mechanism of stellar explosions relies on the extraction of rotational free energy of collapsing progenitor core via magnetic fields. Hence a high angular momentum of the core is preconditioned for facilitating the mechanism [276]. Given (a rapid) rotation of the precollapse core, there are at least two ways to amplify the initial magnetic fields to a dynamically important strength, namely, by the field wrapping by means of differential rotation that naturally develops in the collapsing core, and by the MRI (MRI, see [16]). Each of them we are going to review briefly in the following.

(a) Field-Wrapping Mechanism. Three dimensional plots of Figure 17 are useful to see how the field wrapping occurs. For the 2D model taken from our 2D special relativistic (SR) MHD simulation [268], the precollapse magnetic field is set to be as high as $10^{12} \mathrm{G}$ that is uniform and parallel to the rotational axis and the initial $\beta$ parameter (ratio of initial rotational energy to the absolute value of the initial gravitational energy) is taken to be $0.1 \%$ with a uniform rotation imposed in the iron core. From the left panel, it can be seen that the field lines are strongly twisted around the rotational axis. From the induction equation of ideal MHD equations, the time evolution of the toroidal fields $\left(B_{\phi}\right)$ can be expressed as (e.g., [276]),

$$
\frac{\partial B_{\phi}}{\partial t} \approx B_{X}\left(X \frac{\partial \Omega}{\partial X}\right)
$$

where $X$ denotes the distance from the rotational axis and $B_{X}$ represents the $X$ component of the poloidal fields in the cylindrical coordinates $(X, Z)$. Given that a precollapse iron core has strong magnetic field (such as $B_{X, 0} \sim 10^{12} \mathrm{G}$ with $B_{X, 0}$ representing the initial poloidal fields) and rapid rotation (such as $P_{\text {init }} \lesssim 4$ s with $P_{\text {init }}$ being the precollapse rotation period), the typical amplification of $B_{\phi}$ near core bounce may be estimated as

$$
\Delta B_{\phi} \approx \frac{t}{P_{\text {rot }}} B_{X} \sim 10^{15} \mathrm{G}\left(\frac{t}{100 \mathrm{~ms}}\right)\left(\frac{P_{\text {rot }}}{1 \mathrm{~ms}}\right)\left(\frac{B_{X, 0}}{10^{12} \mathrm{G}}\right),
$$

where $t$ represents the typical timescale when the field amplification via the wrapping processes saturates, $P_{\text {rot }}$ denotes the rotational period near bounce. Equation (2) shows that the toroidal fields grow linearly with time by wrapping the poloidal fields $\left(B_{X}\right)$, which is the so-called $\Omega$ dynamo (e.g., [265]). The $10^{15} \mathrm{G}$ class magnetic fields are already dynamically important strength to affect the postbounce hydrodynamics as will be mentioned below.

The white lines in the right panel in Figure 17 show the streamlines of matter. A fallback of material just outside of the jet head advecting downwards to the equator (like a cocoon) is seen. In this jet with a cocoon-like structure, the magnetic pressure is always dominant over the matter pressure. As estimated above, the typical field strength behind the shock is $\sim 10^{15} \mathrm{G}$. The critical strength of the toroidal magnetic field to induce the magnetic shock revival (i.e., the revival of the stalled bounce shock due to the MHD mechanism) is estimated as follows. The matter in the regions behind the stalled shock is pushed inwards by the ram pressure of the accreting matter. This ram pressure is estimated as,

$$
P=4 \times 10^{28}\left(\frac{\rho}{10^{10} \mathrm{~g} / \mathrm{cm}^{3}}\right)\left(\frac{\Delta v}{2 \times 10^{9} \mathrm{~cm} / \mathrm{s}}\right)^{2} \mathrm{erg} / \mathrm{cm}^{3},
$$

where $\rho$ and $\Delta v$ denote typical density and radial velocity in the vicinity of the stalled shock. When the toroidal magnetic fields are amplified as large as $\sim 10^{15} \mathrm{G}$ due to the field wrapping behind the shock, the resulting magnetic pressure, $B^{2} / 8 \pi \sim\left(10^{15}\right)^{2} / 8 \pi \sim 10^{29} \mathrm{erg} / \mathrm{cm}^{3}$, can overwhelm the ram pressure, leading to the magnetic shock revival (e.g., Figure 18). The onset timescale of the magnetic shock revival is sharply dependent on the precollapse magnetic fields and rotation (e.g., [46]). As the initial field strength is larger with more rapid rotation imposed, the interval from the shock stall to the MHD-driven shock revival becomes shorter. The speed of the jet head is typically mildly relativistic (at most $\sim 0.4 c$ ), with $c$ being the speed of light (see also the right panel of Figure 18). At the shock breakout of the iron core, the explosion energies generally exceed $10^{51} \mathrm{erg}$ for models that produce MHD explosions in a shorter delay after the stall of the bounce shock. These features are in accord with those obtained in more detailed MHD simulations with spectral neutrino transport (e.g., [46, 273]) (see, e.g., [219] for a more detailed comparison).

(b) On the MRI. We are now in a position to discuss possible impacts of the MRI in supernova cores. Akiyama et al. [277] were the first to point out that the interfaces surrounding the nascent PNSs quite generally satisfy the MRI instability criteria. Therefore any seed magnetic fields can be amplified exponentially in the differentially rotating layers, much faster than the linear amplification due to the field wrapping (e.g., (2)). After the MRI enters to the saturated state, the field strength might reach $\sim 10^{15-16} \mathrm{G}$, which is high enough to affect the supernova dynamics. Not only in the field amplification, the MRI plays a crucial role also in operating the MHD turbulence (see $[16,278,279]$ ). The turbulent viscosity sustained by the MRI can convert a fraction of the shear rotational energy to the thermal energy of the system. Thompson et al. [141] suggested that the additional energy input by the viscous heating can help the neutrinodriven supernova explosion. Followed by the exponential field amplification and additional heating, a natural outcome of the magnetorotational core-collapse is expected to be the formation of energetic bipolar explosions, which might be observed as hypernovae (e.g., Section 2.3).

Note again that bipolar explosions obtained in the previous section with the assumption of a strong precollapse magnetic field ( $\left.\gtrsim 10^{12} \mathrm{G}\right)$, are predominantly driven by the field wrapping processes, not by the MRI. Shibata et al. [280] pointed out in their fully 2D GRMHD core-collapse simulations that more than 10 grids are at least needed to capture the fastest growing mode of the MRI (see also [281]). As well-known, the growth rate of MRI-unstable 


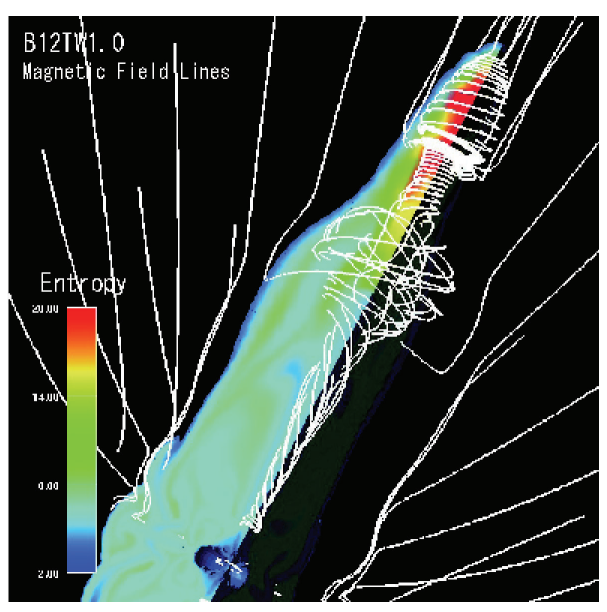

(a)

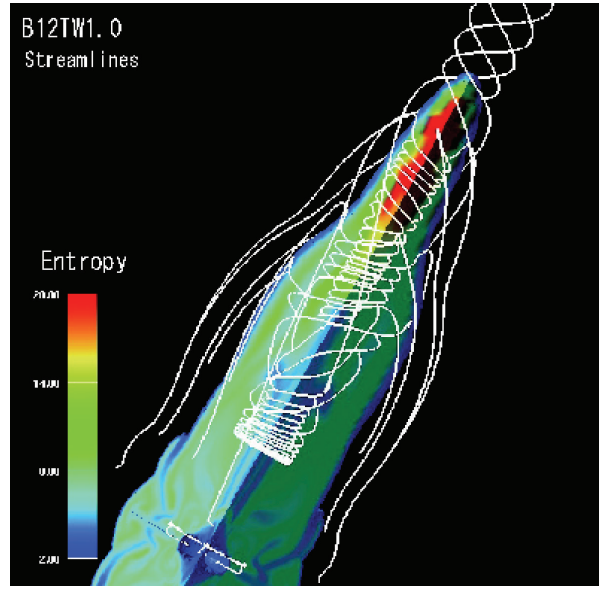

(b)

Figure 17: Three-dimensional plots of entropy with the magnetic field lines (a) and the streamlines of the matter (b) during the jet propagation at $20 \mathrm{~ms}$ and $94 \mathrm{~ms}$ after bounce, respectively (for model B12TW1 taken from [268]). The outer edge of the sphere colored by blue represents the radius of $7.5 \times 10^{7} \mathrm{~cm}$. These panels highlight not only the wound-up magnetic field around the rotational axis (a), but also the fallback of the matter from the jet head of the downwards to the equator, making a cocoon-like structure behind the jet (b).
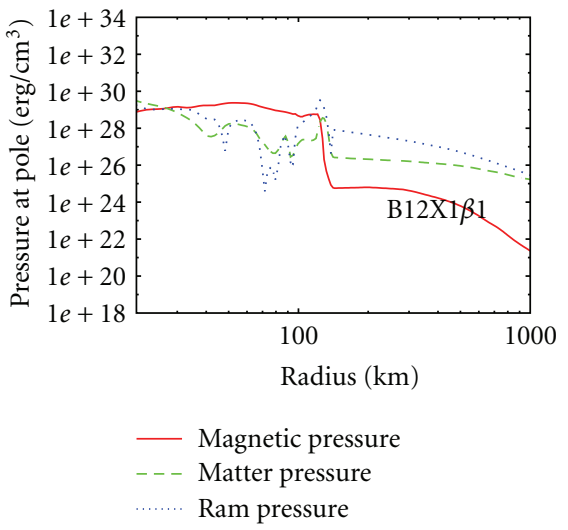

(a)

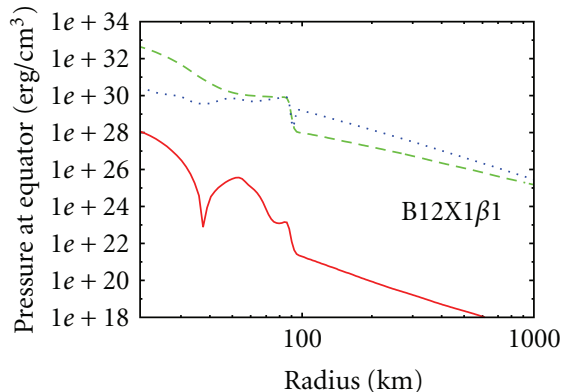

_- Magnetic pressure
-.- Matter pressure
… Ram pressure

(b)

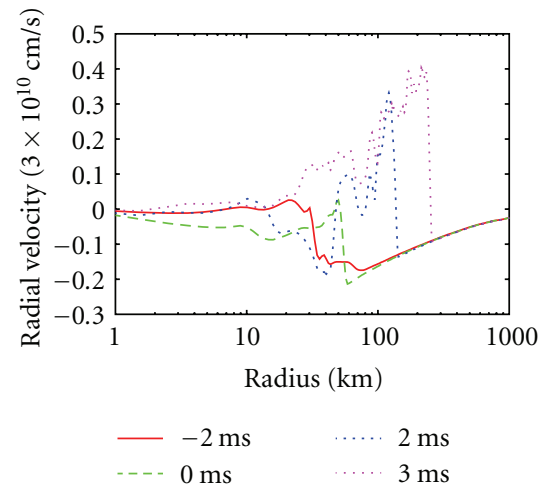

(c)

Figure 18: Left and middle panels show magnetic pressure (red line) versus ram pressure (blue line) for a typical MHD model (same as Figure 17) along the polar axis (a) or the equatorial plane (b) at $2 \mathrm{~ms}$ after the revival of the stalled bounce shock. The matter pressure is shown by green line as a reference. The right panel shows a velocity profile along the pole measured from the shock-stall $(0.0 \mathrm{~ms}$ in the panel). For the equator, the magnetic pressure is much less than the ram pressure (middle panel), while the magnetic pressure amplified by the field wrapping along the pole becomes as high as the ram pressure of the infalling material at the shock front ((a), note that the shock position can be inferred by the discontinuity at around $150 \mathrm{~km}$ ), leading to the MHD-driven shock formation (c). These figures are taken from [219].

modes depend on the product of the field strength and the wave number of the mode [16]. When a rapidly precollapse core is strongly magnetized as $10^{11} \mathrm{G}$ (like in the case of collapsar progenitor), the wavelength is on the order of $\mathrm{km}$ in the postbounce core. In this case, the exponential field growth of the MRI was successfully captured by high-resolution simulations [280]. On the other hand, the fastest growing modes drop below several meters [282] for canonical supernova progenitors, which rotates much more slowly with weaker initial fields $\left(\sim 10^{9} \mathrm{G}\right)$ as predicted by recent stellar evolution models [126, 127]. At present, it is computationally too expensive to resolve those small scales in the global MHD simulations, typically more than two or three orders-of-magnitudes smaller than their typical finest grid size. To reveal the nature of the MRI, local simulations focusing on a small part of the MRI-unstable regions are expected to be quite useful as traditionally studied in the context of accretion disks (see [16]).

Obergaulinger et al. [282] were the first to study the growth and saturation level of the MRI in the supernova 
environment (see, [283] for extensive simulations that focus on the MRI in relativistic outflows in the context of gammaray bursts and active galactic nuclei). To ease a drawback of the local shearing box simulation, they employed the shearing disk boundary conditions by which global radial density stratification can be taken into account. By performing such a semiglobal simulation systematically in $2 \mathrm{D}$ and $3 \mathrm{D}$, they derived scaling laws for the termination of the MRI. As estimated in [277], the MRI was shown to amplify the seed fields exceeding $10^{15} \mathrm{G}$. These important findings may open several questions that motivate us to join in this effort, such as how the nonlinear properties as well as the scaling laws could be in the subsequent nonlinear state and whether the viscous and resistive heating driven by the MRI turbulence could or could not affect the supernova mechanism.

In the following, we briefly summarize the current status of our MRI project, in which we perform a local shearing box simulation to study the MRI-driven turbulence and their nonlinear properties bearing in mind the application to the supernova core (Masada, Takiwaki, and Kotake in preparation). The final goal of this project is to determine how much the conversion from the rotational energy (that the differential rotation taps) to the thermal energy occurs via the MRI turbulence, which was treated parametrically in [141]. As will be shown later, our preliminary results suggest that the additional energy supply would affect the neutrinodriven explosion in the case of rapidly rotating cores at late postbounce phase.

To study the nonlinear properties of the MRI, we solve viscous MHD equations by a finite-difference code that was originally developed by Sano et al. [284]. The hydrodynamic part is based on the second-order Godunov scheme [285], which consists of Lagrangian and remap steps. The exact Riemann solver is modified to account for the effect of tangential magnetic fields. The field evolution is calculated with Consistent MoC-CT method [286]. The energy equation is solved in the conservative form and the viscous terms are consistently calculated in the Lagrangian step. The advantages of our scheme are its robustness for strong shocks and the satisfaction of the divergence-free constraint of magnetic fields $[287,288]$.

Rigorously speaking, 3D simulations are absolutely required for the study of the MRI because only by them the disruption of channel structures, mode couplings, and the growth of parasitic instabilities can be accurately determined (e.g., $[289,290])$. But in the following, we are only able to show our $2 \mathrm{D}$ results assuming axisymmetry, which are currently being updated to $3 \mathrm{D}$. Bearing these caveats in mind, we briefly illustrate fundamental properties of the MRI (which is not always familiar with supernova modellers,) and discuss their possible impacts on the explosion dynamics.

(c) Local Simulations in Axisymmetry. As it is well known, there are three typical evolutionary stages observed in our local simulations. They are (i) exponential growth stage, (ii) transition stage, and (iii) nonlinear turbulent stage [278, 291]. Each stage is denoted by white, dark gray, and light gray shaded regions in Figure 19.
In the stage (i), the channel structure of the magnetic field exponentially evolves and inversely cascades to the larger spatial scale with small structures merging as the magnetic field is amplified. This is because the channel modes of the MRI are an exact solution for the nonlinear MHD equations [292]. The temporal evolution of channel structures for the toroidal magnetic field and their inversely cascading nature are shown in panels (a) and (b) of Figure 20. Then in the stage (ii), the channel structure of the magnetic field is disrupted via the parasitic instability and magnetic reconnection at the transition stage [282,292]. The channel disruption induces a drastic phase-shift from the coherent structure to the turbulent tangled structure of the field as is illustrated by panel (c) of Figure 20. The magnetic energy stored in the amplified magnetic field is then converted to the thermal energy of the system. Finally in the stage (iii), the nonlinear stage emerges after the channel structures are disrupted to be a turbulence state driven by the MRI (see panel (d) in Figure 20). Figure 19(a) shows that, at this stage, the turbulent Maxwell stress $\left(M_{r \phi}=\right.$ $\left.-B_{r} B_{\phi} / 4 \pi\right)$ dominates over the Reynolds stress $\left(R_{r \phi}=\right.$ $\left.\rho v_{r} \delta v_{\phi}\right)$. Therefore the turbulent Maxwell stress is the main player to convert the free energy stored in the differential rotation into the thermal energy of the system.

Figure 19(b) shows the power spectra of the magnetic energy in the nonlinear turbulent stage. The logarithmic slope of the spectrum roughly coincides with $-11 / 3$ in the regime $10 \lesssim \bar{k} \lesssim 100$ while it steepens at higher wavenumbers where numerical effects become important (like $\left.k^{-6}\right)$. This indicates that the turbulent state in the MRI can be represented by the Kolmogorov spectrum for a homogeneous, incompressible turbulence that scales as $k^{-11 / 3}$. These features would be consistent with the 3D properties of the MRI-driven turbulence [278]. It has been pointed out in the previous axisymmetric local shearing box simulation that the channel structures do neither disrupt nor transit to the turbulent state without a relatively strong resistive effect $[282,293]$. We however adopted the simulation box with a large aspect ratio $\left(L_{x} / L_{z}=4\right)$ and quasi-isothermal equation of state $(\gamma \simeq 1)$, both of which could facilitate to disrupt the channel structures [294] as in 3D simulations.

Figure 21 shows the time- and volume-averaged Maxwell (green squares) and Reynolds stresses (orange diamonds, panel (a)), and magnetic energy (blue circles, panel (b)) in the turbulent stage as a function of the initial magnetic energy. It is shown that the Maxwell stress depends on the initial magnetic flux and it provides the following scaling relation:

$$
\left\langle\left\langle M_{r \phi}\right\rangle\right\rangle \propto E_{\mathrm{mag}, \mathrm{ini}},
$$

with $E_{\text {mag,ini }} \equiv B_{\mathrm{ini}}^{2} / 8 \pi$. This suggests that the magnetic flux initially penetrating the system controls the nonlinear transport properties of the MRI-driven turbulence. This is qualitatively consistent with the previous results in the accretion disk [278, 284, 295, 296] while the power-law index obtained here is slightly larger. Note that the magnetic energy at the nonlinear turbulent stage has a linear dependence on the initial magnetic flux (because $\left\langle\left\langle E_{\text {mag }}\right\rangle\right\rangle \propto E_{\text {mag,ini }}^{1 / 2} \propto B_{\text {ini }}$ 


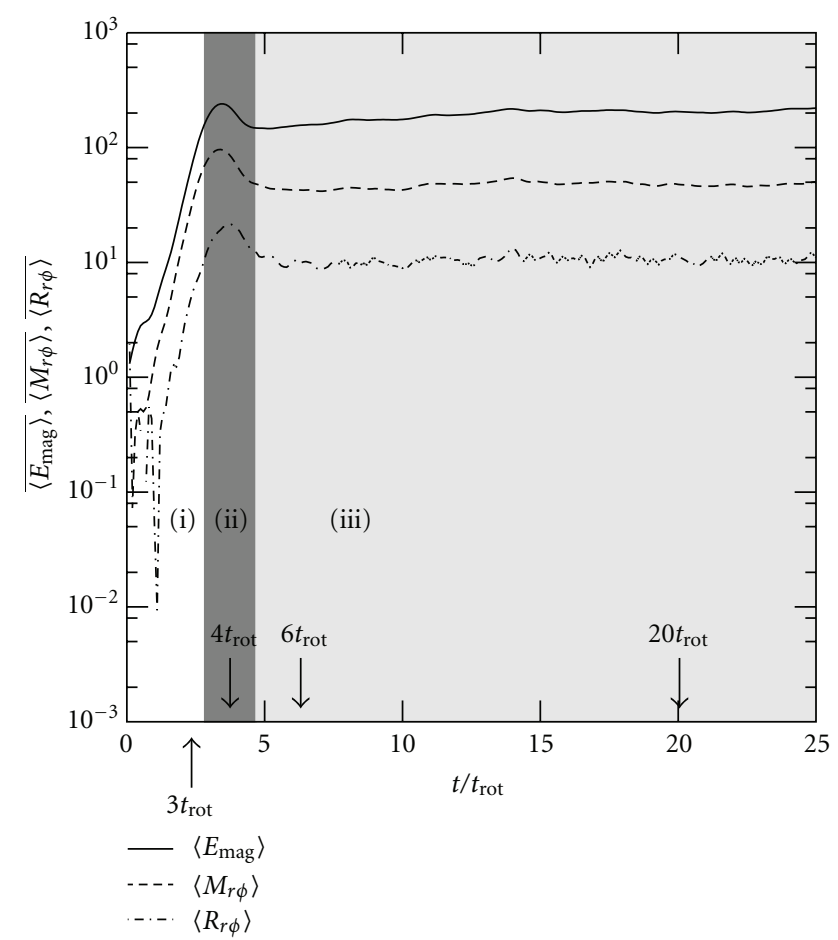

(a)

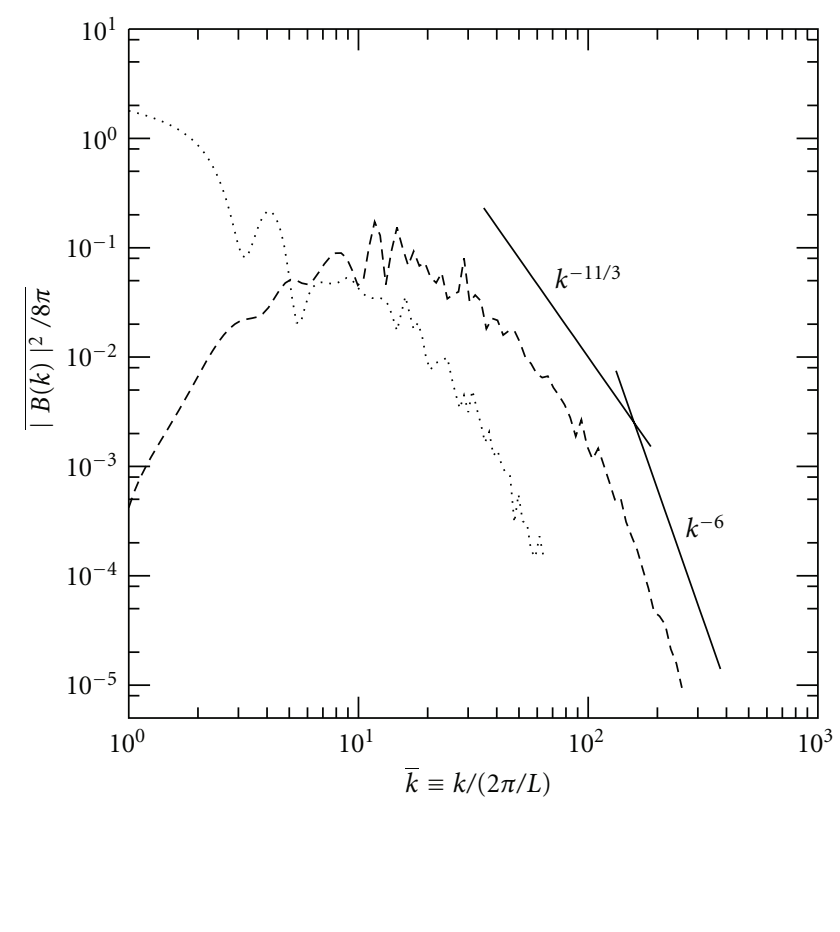

(b)

FIGURE 19: (a) Temporal evolution of the volume averaged magnetic energy, Maxwell and Reynolds stresses in the fiducial run are represented by thick, dashed, and dash-dotted curves, respectively. The vertical axis is normalized by the initial magnetic energy $E_{\text {mag, } 0} \equiv B_{0}^{2} / 8 \pi$, that is $\overline{\left\langle E_{\mathrm{mag}}\right\rangle} \equiv\left\langle E_{\mathrm{mag}}\right\rangle / E_{\mathrm{mag}, 0}, \overline{\left\langle M_{r \phi}\right\rangle} \equiv\left\langle M_{r \phi}\right\rangle / E_{\mathrm{mag}, 0}$, and $\overline{\left\langle R_{r \phi}\right\rangle} \equiv\left\langle R_{r \phi}\right\rangle / E_{\mathrm{mag}, 0}$ where single bracket denotes the volume average of the physical variables. Horizontal axis is normalized by the rotational period $t_{\text {rot }} \equiv 2 \pi / \Omega$. Three typical evolutionary stages, (i) exponential growth stage, (ii) transition stage, and (iii) nonlinear turbulent stage, are denoted by white, dark gray, and light gray shaded regions. (b) The amplitude of power spectra of the magnetic energy $|B(k)|^{2}$ along the $k_{x}$ and $k_{z}$ axes. The dashed and dotted curves describes the $k_{x}$ and $k_{z}$ components respectively. The vertical axis is normalized by the initial magnetic energy $E_{\mathrm{mag}, 0}$. The wave-numbers shown in the horizontal axis are normalized by the $2 \pi / L$. The upper thick curve demonstrates the Kolmogorov slope for isotropic incompressible turbulence $\propto k^{-11 / 3}$. The lower slope is proportional to $k^{-6}$ just for the reference. In our local simulations, we prepare a 2D shearing box which is threaded by the initial vertical field $B_{0}=1.4 \times 10^{12} \mathrm{G}$ with imposing initial gas pressure $P_{0}=4 \times 10^{28} \mathrm{dyn} \mathrm{cm}^{-2}$, angular velocity $\Omega_{0}=2000 \mathrm{sec}^{-1}$ and shear parameter $q_{0}=1.25$, respectively. Here the shear parameter represents the degree of differential rotation as $q \equiv-d \ln \Omega / d \ln r$ with $r$ being the radial coordinate. The other parameters are fixed to mimic the properties in the vicinity of the PNS's surface in the postbounce phase (i.e., the density is taken to be $\rho_{0}=10^{12} \mathrm{~g} \mathrm{~cm}^{-3}$ and the box size is taken to be $L_{x}=4 \mathrm{~km}$ (horizontal) $\times L_{z}=1 \mathrm{~km}(\mathrm{vertical})$ ).

(Figure 21(b))). This is also qualitatively consistent with Hawley et al. [278]. In this way, we investigated parameter dependence of the rotational shear ( $q$ parameter) as well as the initial pressure $(P)$ on the saturated value of the Maxwell stress and deduced a scaling relation that yields $M_{r \phi} \propto$ $B^{2} P^{1 / 4}[q /(2-q)]^{1 / 4} \Omega^{1 / 2}$. Here the shear parameter represents the degree of differential rotation as $q \equiv-d \ln \Omega / d \ln r$ with $r$ being the radial coordinate.

Using the scaling relation obtained in our local simulations, the heating rate maintained by the MRI turbulence can be estimated as:

$$
\begin{aligned}
\epsilon_{\mathrm{MRI}} & =M_{r \phi} q \Omega \\
& =\left[f\left(\frac{B_{\mathrm{pb}}}{B_{0}}\right)^{2}\left(\frac{P_{\mathrm{pb}}}{P_{0}}\right)^{1 / 4} s_{\mathrm{pb}}^{1 / 4}\left(\frac{\Omega_{\mathrm{pb}}}{\Omega_{0}}\right)^{1 / 2}\right] q \Omega \\
& \simeq 10^{30} \mathrm{erg} \mathrm{cm}^{-3} \mathrm{sec}^{-1}
\end{aligned}
$$

$$
\times f_{30} q_{\mathrm{pb}, 1} s_{\mathrm{pb}, 1}^{1 / 4}\left(\frac{B_{\mathrm{pb}}}{10 B_{0}}\right)^{2}\left(\frac{P_{\mathrm{pb}}}{100 P_{0}}\right)^{1 / 4}\left(\frac{\Omega_{\mathrm{pb}}}{\Omega_{0}}\right)^{3 / 2}
$$

where $f_{30}$ represents a typical amplification factor of the stress normalized by 30 (e.g., Figure 21 ), $q_{\mathrm{pb}, 1}$ is the shear rate normalized by unity, and $s_{\mathrm{pb}, 1}$ is the ratio of the vorticity to the shear normalized by unity. Since we are interested in the growth of the MRI in the postbounce phase, the original magnetic flux, gas pressure angular velocity, and the shear rate $B_{0}=1.4 \times 10^{12} \mathrm{G}, P_{0}=4 \times 10^{28} \mathrm{erg} \mathrm{cm}^{-3}, \Omega_{0}=$ $2000 \mathrm{rad} \mathrm{s}^{-1}$, and $q=1.25$ are replaced by $B_{\mathrm{pb}}, P_{\mathrm{pb}}, \Omega_{\mathrm{pb}}$ and $q_{\mathrm{pb}}$ with some amplification factors.

A typical volume in which the MRI-driven turbulence is active, may be estimated as $V_{\mathrm{MRI}}=4 \pi R^{2} h \simeq 10^{21} \mathrm{~cm}^{3} R_{7}^{2} h_{6}$, where $R_{7}=R / 10^{7} \mathrm{~cm}$ is typical radius of the PNS normalized by $10^{7} \mathrm{~cm}$, and $h_{6}=h / 10^{6} \mathrm{~cm}$ is the typical radial width of 


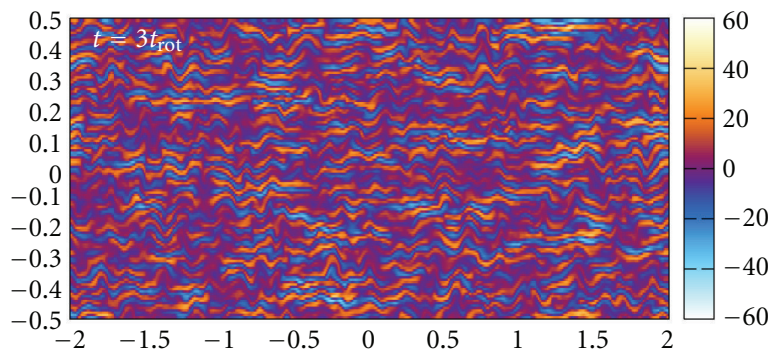

(a)

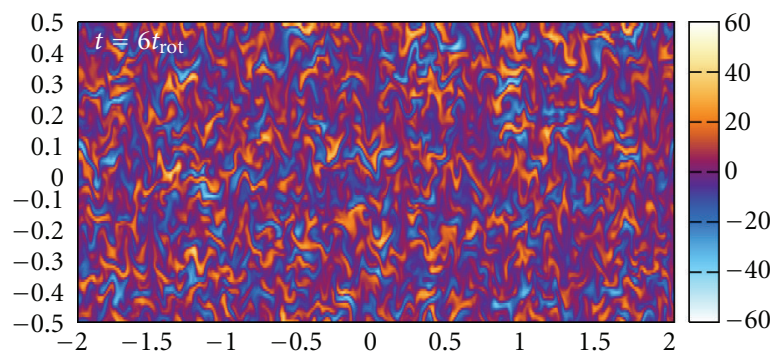

(c)

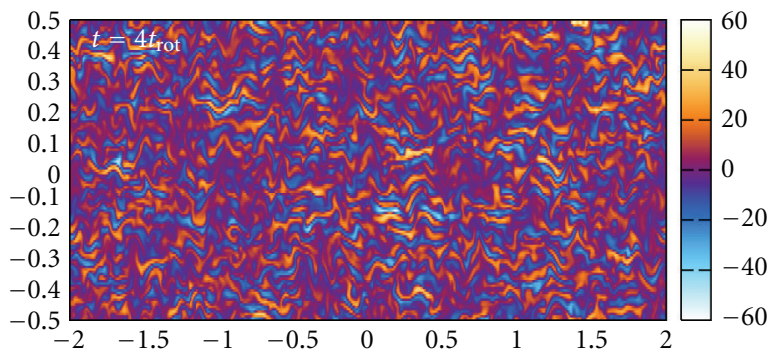

(b)

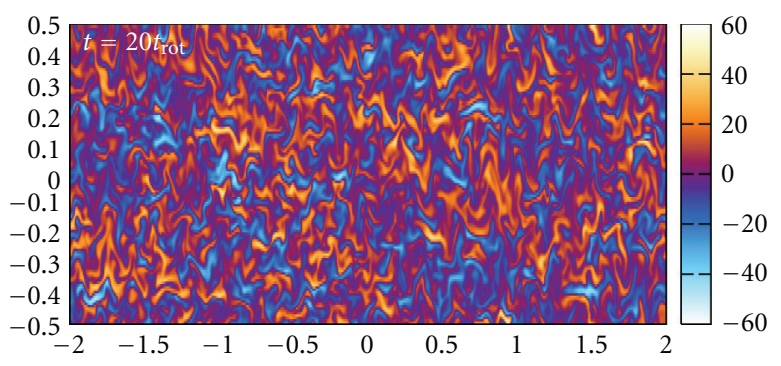

(d)

FIGURE 20: Four snapshots representing the temporal evolution of MRI from the linear to the nonlinear phase. Panels (a), (b), (c) and (d) correspond to the time slice $t=3 t_{\text {rot }}, 4 t_{\text {rot }}, 6 t_{\text {rot }}$, and $20 t_{\text {rot }}$ respectively. The color bar indicates the size of the toroidal magnetic field normalized by the strength of the initial vertical magnetic field $\left(B_{0}=1.4 \times 10^{12} \mathrm{G}\right)$. Note that the vertical and horizontal are normalized by the typical spatial scale of the local portion in the protoneutron stars which is selected as $L=1 \mathrm{~km}$ in the fiducial run. Three typical evolutionary stages are observed, (i) exponential growth stage, (ii) transition stage, and (iii) nonlinear turbulent stage (compare with Figure 19).

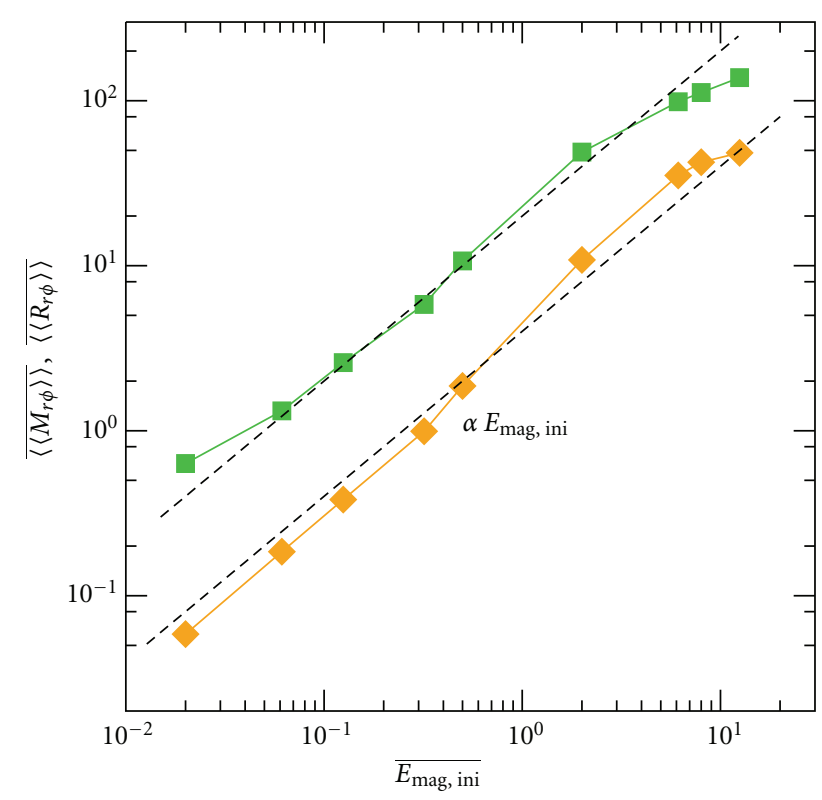

(a)

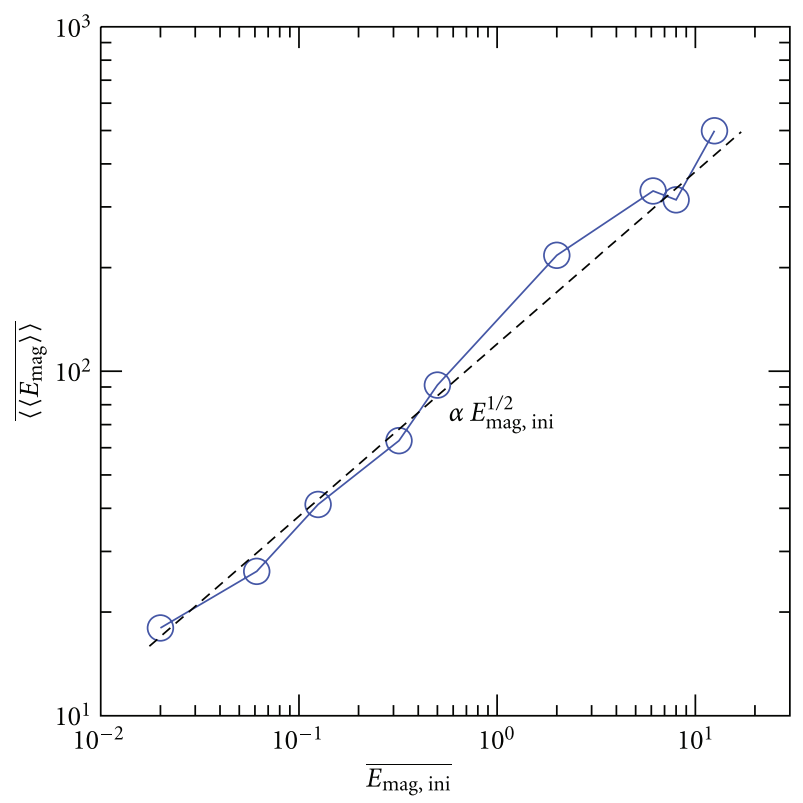

(b)

Figure 21: The dependence of the time- and volume-averaged (a) Maxwell (green squares) and Reynolds stresses (orange diamonds) and (b) magnetic energy (blue circles) and in the turbulent state on the strength of the initial magnetic energy $E_{\text {ini }}$. Both the vertical and horizontal axes are normalized by the initial magnetic energy of the fiducial model $E_{\operatorname{mag}, 0}$. The initial field strength is varied from $\sim 10^{11}$ to $\sim 6 \times 10^{12} \mathrm{G}$. The dashed lines denote reference slopes proportional to (a) $E_{\text {mag }}^{1 / 2}$ and (b) $E_{\text {mag }}$, respectively. We take the time-average of volume-averaged quantities during $90 t_{\text {rot }}<t<100 t_{\text {rot }}$. 


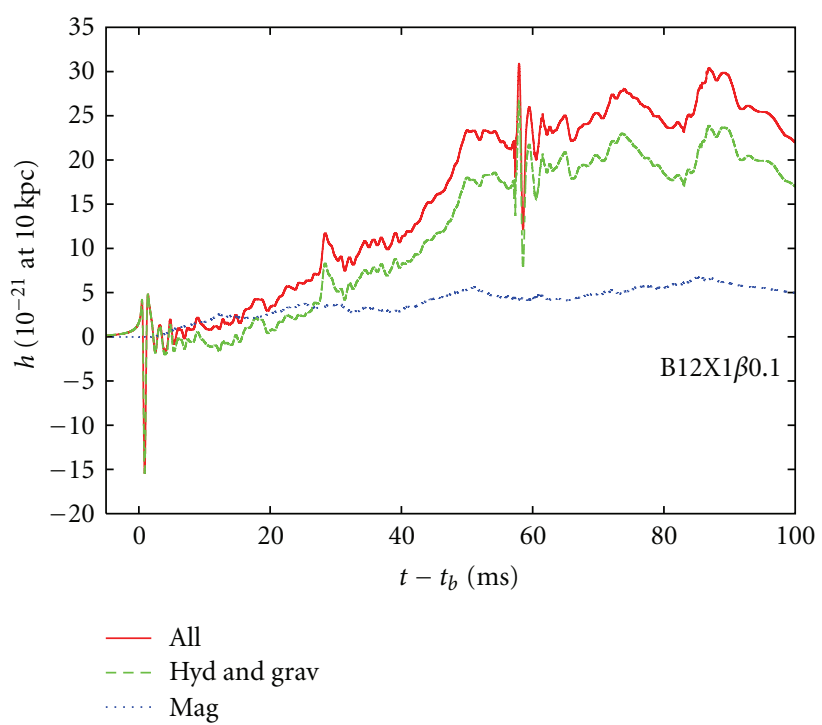

(a)

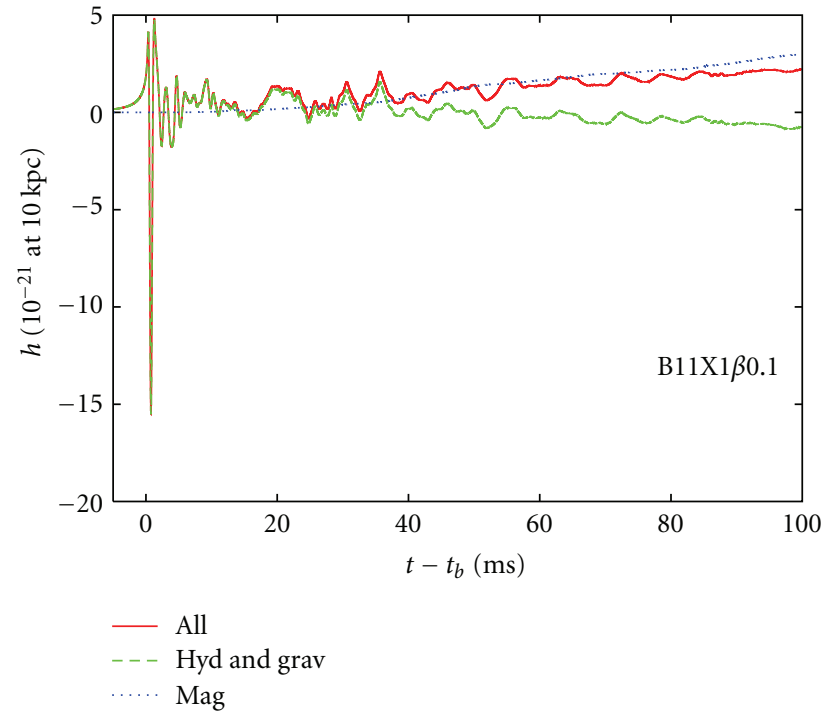

(b)

FIGURE 22: Gravitational waveforms with an increasing trend (left) or not (right panel). Initial rotation parameter is set to be $\beta=0.1 \%$, while the precollapse magnetic field is taken as $10^{12} \mathrm{G}$ (left panel) and $10^{11} \mathrm{G}$ (right panel), respectively (from [219]). The total wave amplitudes are shown by the red line, while the contribution from the magnetic fields and from the sum of hydrodynamic and gravitational parts are shown by blue and green lines, respectively (e.g., (14) and (11), (13)). Note that the bounce GW signals $\left(\left(t-t_{b} \lesssim 20\right.\right.$ ms) are not affected by the magnetic fields significantly, and they are categorized into the so-called type I or II waveforms.

the MRI-active layer normalized by $10^{6} \mathrm{~cm}$. Then the energy releasing rate $L_{\mathrm{MRI}} \equiv \epsilon_{\mathrm{MRI}} V_{\mathrm{MRI}}$ becomes:

$$
\begin{aligned}
L_{\mathrm{MRI}} \sim & 10^{51} R_{7}^{2} h_{6} \mathrm{erg} \mathrm{sec}^{-1} \\
& \times f_{30} q_{\mathrm{pb}, 1} s_{\mathrm{pb}, 1}^{1 / 4}\left(\frac{B_{\mathrm{pb}}}{10 B_{0}}\right)^{2}\left(\frac{P_{\mathrm{pb}}}{100 P_{0}}\right)^{1 / 4}\left(\frac{\Omega_{\mathrm{pb}}}{\Omega_{0}}\right)^{3 / 2} .
\end{aligned}
$$

Typical timescales of neutrino-driven explosions observed in recent supernova simulations are $\gtrsim 400 \mathrm{~ms}$ after bounce (e.g., $[125,138])$. So the energy deposition could be as high as $10^{50} \mathrm{erg}$ if the core rotates rather rapidly as taken in the above estimation. If this is the case, the turbulent heating due to the MRI is expected to play an important role for assisting the neutrino-driven explosion.

It should be noted that nonaxisymmetric modes of the parasitic instability could disrupt the channel structures more effectively than in $2 \mathrm{D}[289,290]$. To obtain more accurate estimate of the amplification factor of $f(6)$, $3 \mathrm{D}$ simulations are unquestionably required, which we are currently undertaking [297].

3.1. Gravitational Waves. As already mentioned in Section 2.2, rapid rotation, necessary for producing strong bounce GW signals, is likely to obtain $\sim 1 \%$ of massive star population (e.g., [62]). This can be really the case, albeit minor, for progenitors of rapidly rotating metal-poor stars, which experience the so-called chemically homogeneous evolution $[274,275]$. In such a case, the MHD mechanism could work to produce energetic explosions, which are receiving great attention recently as a possible relevance to magnetars and collapsars (e.g., [298, 299], see [273, 300, 301] for collective references), which are presumably linked to the formation of long-duration gamma-ray bursts (GRBs) (e.g., [302] for review).

Among the previous studies focusing on the bounce signals (e.g., references in Section 2.2), only a small portion of papers has been spent on determining the GW signals in the MHD mechanism $[175,260,264,265,269,280]$. This may be because the MHD effects on the dynamics as well as their influence over the GW signals can be visible only for cores with precollapse magnetic fields over $B_{0} \gtrsim 10^{12} \mathrm{G}[175$, 264]. Considering that the typical magnetic-field strength of GRB progenitors is at most $\sim 10^{11-12} \mathrm{G}$ [274], this is already an extreme situation. In a more extremely case of $B_{0} \sim 10^{13} \mathrm{G}$, a secularly growing feature in the waveforms was observed $[263,269,280]$. In the following, we summarize the GW signatures based on our 2D SRMHD simulations [219] and discuss how a peculiarity of the MHD mechanism could be imprinted in the GW signals.

The left panel of Figure 22 shows the gravitational waveform with the increasing trend, which is obtained for a model with strong precollapse magnetic field $\left(B_{0}=10^{12} \mathrm{G}\right)$ also with rapid rotation initially imposed $(\beta$ parameter $=$ $0.1 \%)$. Such a feature cannot be observed for a weakly magnetized model $\left(B_{0}=10^{11} \mathrm{G}\right.$, right panel $)$. To understand the origin of the increasing trend, it is straightforward to look into the quadrupole GW formula, which can be expressed as

$$
h_{i j}^{T T}(\mathbf{X}, t) \stackrel{\ell=2, m=0}{=} \frac{1}{R} A_{20}^{E 2}\left(t-\frac{R}{c}\right) T_{i j}^{E 2,20}(\theta, \phi),
$$

where $T_{i j}^{E 2,20}(\theta, \phi)$ is

$$
T_{i j}^{E 2,20}(\theta, \phi)=\frac{1}{8} \sqrt{\frac{15}{\pi}} \sin ^{2} \theta,
$$


(e.g., [303]). $A_{20}^{E 2}$ can be expressed as

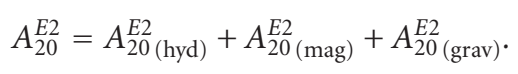

On the right hand side, the first term is related to anisotropic kinetic energies which we call as hydrodynamic part,

$$
\begin{aligned}
A_{20}^{E 2} \text { (hyd) }= & \frac{G}{c^{4}} \frac{32 \pi^{3 / 2}}{\sqrt{15}} \int_{0}^{1} d \mu \int_{0}^{\infty} r^{2} d r f_{20 \text { (hyd) }}^{E 2}, \\
f_{20}^{E 2} \text { (hyd) }= & \rho_{*} W^{2}\left(v_{r}^{2}\left(3 \mu^{2}-1\right)+v_{\theta}^{2}\left(2-3 \mu^{2}\right)-v_{\phi}^{2}\right. \\
& \left.\quad-6 v_{r} v_{\theta} \mu \sqrt{1-\mu^{2}}\right),
\end{aligned}
$$

the second term is related to anisotropy in gravitational potentials which we call as the gravitational part,

$$
\begin{aligned}
A_{20}^{E 2} \text { (grav) }= & \frac{G}{c^{4}} \frac{32 \pi^{3 / 2}}{\sqrt{15}} \int_{0}^{1} d \mu \int_{0}^{\infty} r^{2} d r f_{20 \text { (grav) }}^{E 2}, \\
f_{20 \text { (grav) }}^{E 2}= & {\left[\rho h\left(W^{2}+\left(\frac{v_{k}}{c}\right)^{2}\right)+\frac{2}{c^{2}}\left(p+\frac{|b|^{2}}{2}\right)\right.} \\
& \left.-\frac{1}{c^{2}}\left(\left(b^{0}\right)^{2}+\left(b_{k}\right)^{2}\right)\right] \\
& \times\left[-r \partial_{r} \Phi\left(3 \mu^{2}-1\right)+3 \partial_{\theta} \Phi \mu \sqrt{1-\mu^{2}}\right]
\end{aligned}
$$

and finally the third term is related to anisotropy in magnetic energies that we refer to as magnetic part,

$$
\begin{aligned}
A_{20}^{E 2(\mathrm{mag})}= & -\frac{G}{c^{4}} \frac{32 \pi^{3 / 2}}{\sqrt{15}} \int_{0}^{1} d \mu \int_{0}^{\infty} r^{2} d r f_{20(\mathrm{mag})}^{E 2}, \\
f_{20}^{E 2}(\mathrm{mag})= & {\left[b_{r}^{2}\left(3 \mu^{2}-1\right)+b_{\theta}^{2}\left(2-3 \mu^{2}\right)-b_{\phi}^{2}\right.} \\
& \left.-6 b_{r} b_{\theta} \mu \sqrt{1-\mu^{2}}\right] .
\end{aligned}
$$

The right panel of Figure 23 shows contributions to the total GW amplitudes (6) for the strongly magnetized model (left panel of Figure 22), in which the left-hand-side panels are for the sum of the hydrodynamic and gravitational part, namely $\log \left( \pm\left[f_{20}^{E 2}\right.\right.$ (hyd) $+f_{20}^{E 2}$ (grav) $\left.]\right)$ (left top $(+) / \operatorname{bottom}(-)$ (11), (13), and the right-hand-side panels are for the magnetic part, namely $\log \left( \pm f_{20}^{\mathrm{E} 2}(\mathrm{mag})\right)$ (right top $(+) /$ bottom $(-)$ ) (e.g., (14)). By comparing the top two panels in Figure 19, it can be seen that the positive contribution is overlapped with the regions where the MHD outflows exist. The major positive contribution is from the kinetic term of the MHD outflows with large radial velocities (e.g., $+\rho_{*} W^{2} v_{r}^{2}$ in (11)). The magnetic part also contributes to the positive trend (see top right-half in the right panel (labeled by mag $(+))$ ). This comes from the toroidal magnetic fields (e.g., $+b_{\phi}^{2}$ in (14)), which dominantly contribute to drive MHD explosions.

Figure 24 shows the GW spectra for a pair models of B12X5 $\beta 1$ and B11X5 $\beta 1$ like in Figure 22 that with or without the increasing trend. Regardless of the presence (left panel) or absence (right panel) of the increasing trend, the peak amplitudes in the spectra are around $1 \mathrm{kHz}$. This is because they come from the GWs near at bounce when the MHD effects are minor. On the other hand, the spectra for lower frequency domains (below $\sim 100 \mathrm{~Hz}$ ) are much larger for the model with the increasing trend (left panel) than without (right panel). This reflects a slower temporal variation of the secular drift inherent to the increase-type waveforms (e.g., Figure 22). It is true that the GWs in the low frequency domains mentioned above are relatively difficult to detect due to seismic noises, but a recently proposed future space interferometers like Fabry-Perot type DECIGO is designed to be sensitive in the frequency regimes [217, 305] (e.g., the black line in Figure 24). Our results suggest that these low-frequency signals, if observed, could be one important messenger of the increase-type waveforms that are likely to be associated with MHD explosions exceeding $10^{51} \mathrm{erg}$.

3.2. Neutrino Signals. As of now, SN1987A remains the only astrophysical neutrino source outside of our solar system. Even though the detected events were just two dozen, these events have been studied extensively (yielding $\sim 500$ papers) and have allowed us to have a confidence that our basic picture of the supernova physics is correct (e.g., [4], see $[5,6]$ for a recent review). For a next nearby event, it is almost certain that the flagship detectors like Super-Kamiokande and IceCube are able to detect a SN "neutrino light curve" with high statistics. For the detectors, the horizon to the sources now extends out to about $100 \mathrm{kpc}$ and thus covers our galaxy and its satellites. A future megaton-class detector reaches as far as Andromeda galaxy at a distance of $780 \mathrm{kpc}$ (e.g., Hyper-Kamiokande, Memphys, and LBNE) and largescale scintillator (e.g., HALO [307] that is an upgrade of SNO) or liquid-Argon detectors (GLACIER [308] that is an upgrade of ICARUS) will also play an important role (see [309]) for a complete list and details).

Before core bounce, neutrinos of different flavors are initially tapped in their relative neutrino spheres with increasing their Fermi energies as the central density becomes higher. After bounce, the shock dissociates nuclei into free nucleon, which drastically increases the number of protons, leading to a sharp increase in electron capture and production of the prompt $v_{e}$ burst. At this neutronization epoch (in the first $10-20 \mathrm{~ms}$ postbounce), the largest difference in the lightcurves among $v_{e}$ and the rest of the neutrino species can be seen (e.g., Figure 1 in [310]). Since neutrino oscillations take place only when there is a difference in the neutrino fluxes among different species (see Section 3 [22] for more details on the neutrino oscillations), the oscillation effects in the neutronization epoch would be strongest. Unfortunately, however, they are very hard to measure because the currently-running detectors are primarily sensitive to $\bar{v}_{e}$ signals that are produced by the inverse beta process $\bar{v}_{e}+p \rightarrow$ $e^{+}+n$. It should be noted that the detection the $v_{e}$ bursts is important for studying not only neutrino oscillations but also for determining the direction and distance to the source [310]. In a megaton-class Čherenkov detector in which gadolinium is added to catch neutrons [311], and large liquid-Argon detectors (like ICARUS and GLACIER cited above) are expected to be powerful $v_{e}$ detectors.

During the subsequent accretion phase (approximately before $1 \mathrm{~s}$ postbounce), the bounce shock stagnates and matter falls in through the stalled shock to the center, 


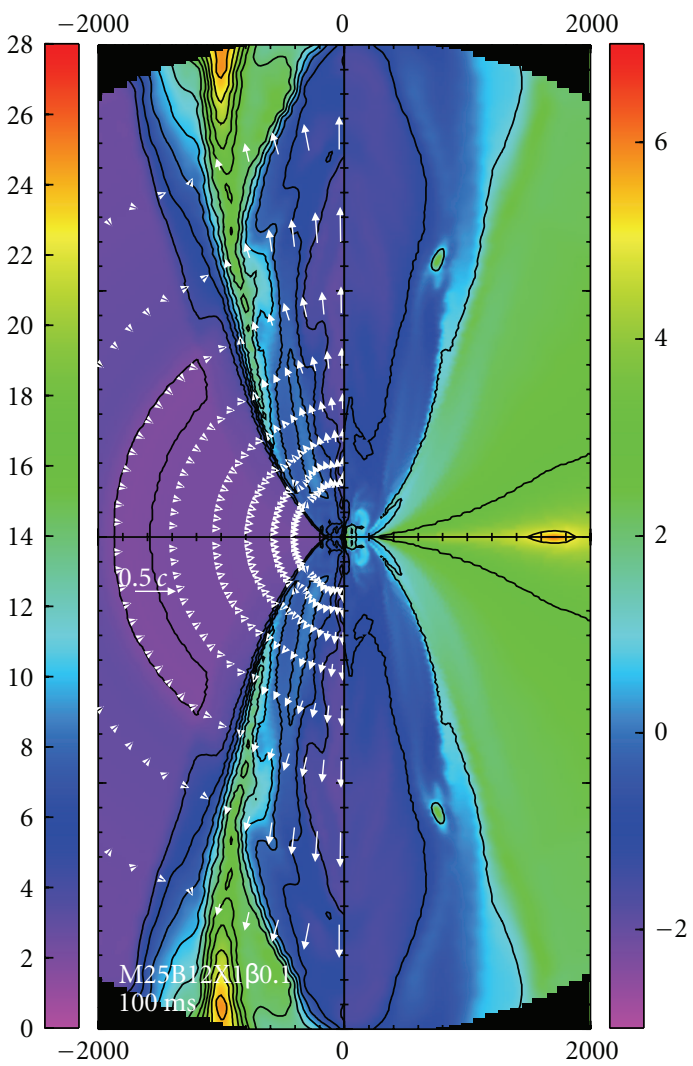

(a)

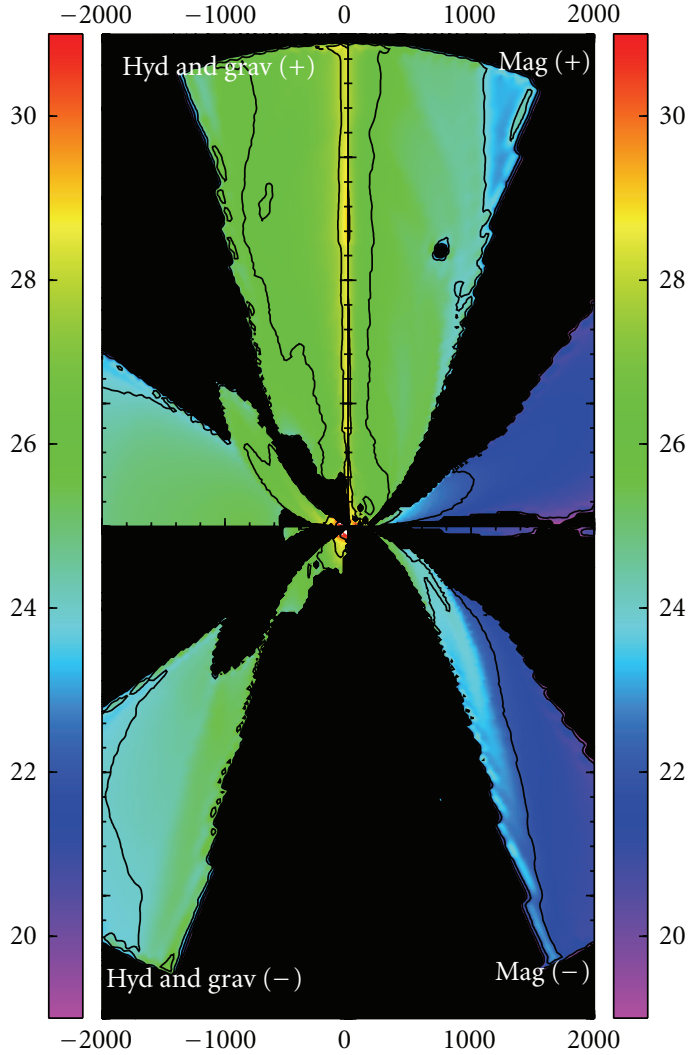

(b)

Figure 23: Left panel shows distributions of entropy ( $k_{B} /$ baryon) (left) and logarithm of plasma $\beta$ (right) for model B12X1 $\beta 0.1$ at 100 ms after bounce. The white arrows in the left-hand side show the velocity fields, which are normalized by the scale in the middle left edge $(0.5 c)$. Right panel shows the sum of the hydrodynamic and gravitational parts (indicated by "hyd and grav" in the left-hand side) and the magnetic part (indicated by "mag" in the right-hand side), respectively. The top and bottom panels represent the positive and negative contribution (indicated by $(+)$ or $(-))$ to $A_{20}^{E 2}$, respectively (see text for more detail). The side length of each plot is $4000(\mathrm{~km}) \times 8000(\mathrm{~km})$. This figure is taken from [219].

releasing gravitational energy that powers neutrino emission. For relatively well-studied progenitors in the mass range of 10.8 to $25 M_{\odot}$ (e.g., $[96,121,310,312,313]$ and references therein), one generally finds $L_{\nu_{e}} \sim L_{\bar{\nu}_{e}}>L_{v_{x}}$ and $\left\langle E_{v_{x}}\right\rangle>$ $\left\langle E_{\bar{v}_{e}}\right\rangle \gtrsim\left\langle E_{v_{e}}\right\rangle$ with $L_{\gamma_{i}}$ and $\left\langle E_{\gamma_{i}}\right\rangle$ representing luminosity and average neutrino energy for the neutrino species of $i=v_{e}, \bar{v}_{e}, v_{x}\left(; v_{\mu}, v_{\tau}\right.$ and their antiparticles). The accretion phase is followed by the PNS (protoneutron star) cooling in which the accretion stops and the PNS settles to become a neutron star after a successful revival of the stalled bounce shock into explosions. Since the explosion mechanism is still under debate (see discussion in Section 2), it is at present difficult to tell the accurate turn-over time. But it should be less than 1-2 s postbounce, otherwise the central PNS would collapse into a black hole [314]. In the PNS cooling phase, the luminosities of all neutrino species become similar $L_{\nu_{e}} \sim$ $L_{\bar{\gamma}_{e}} \sim L_{\gamma_{x}}$ (with the energy hierarchy of $\left\langle E_{\gamma_{x}}\right\rangle>\left\langle E_{\bar{\gamma}_{e}}\right\rangle>\left\langle E_{\gamma_{e}}\right\rangle$ ) and decrease monotonically with time. Due to the similarity in the luminosities and spectra among $\bar{v}_{e}$ and $v_{x}$ and also due to the darkening with time, it is much harder to see flavor oscillation effects by the currently running detectors.

Neutrinos streaming out of the iron core interact with matter via the Mikheyev-Smirnov-Wolfenstein (MSW) effect (e.g., [78]) firstly in propagating through progenitor envelope and then through the Earth before reaching detectors. Such effects have been extensively investigated so far from various points of view, with a focus such as on the progenitor dependence of the early neutrino burst (e.g., $[310,315])$ and on the Earth matter effects (e.g., [316, 317]). The conversion efficiency via the MSW effect depends sharply on density, equivalently electron fraction gradients, thus sensitive to discontinuities produced by the passage of supernova shocks. Such shock effects have been also extensively studied (e.g., [318-322], see [79] for a recent review). The shock passage to the so-called high-resonance regions may be observed as a sudden decrease in $v_{e}$ events in the case of normal mass hierarchy (or $\bar{\nu}_{e}$ in the case of inverted mass hierarchy). Such features monitoring time evolution of the density profile like 


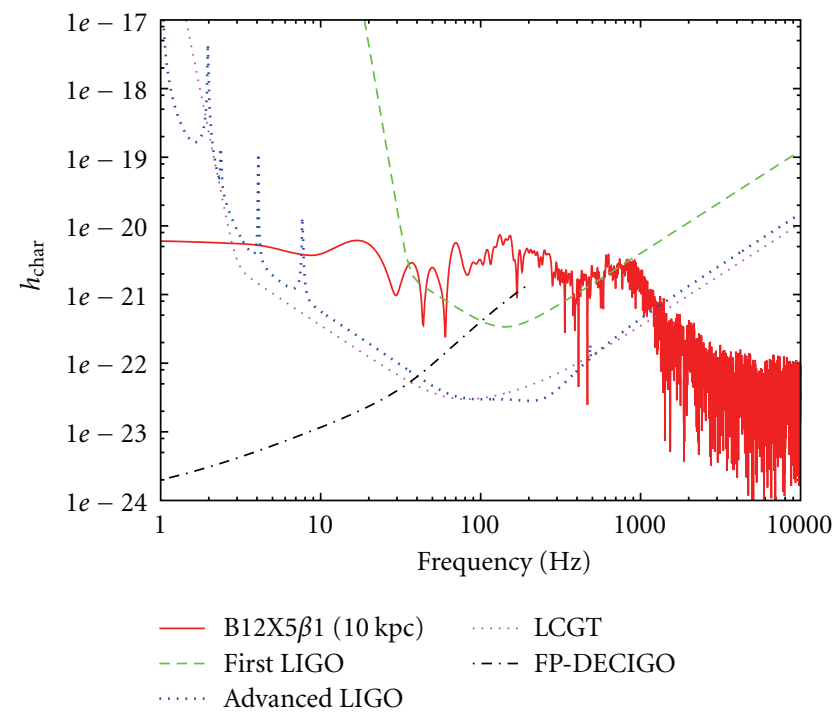

(a)

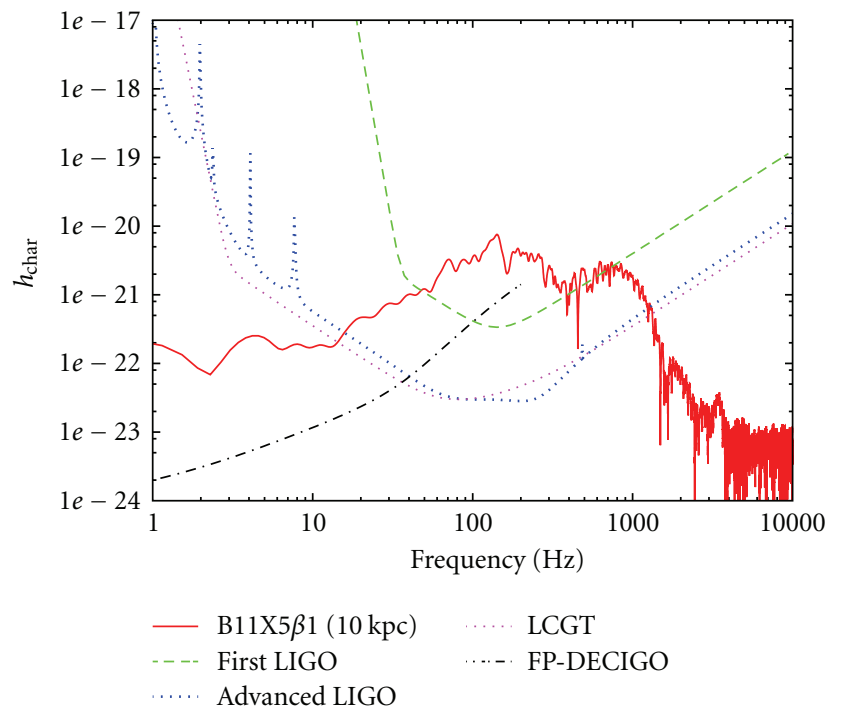

(b)

FIGURE 24: Gravitational-wave spectrum for representative models with the expected detection limits of the first LIGO [11], the advanced LIGO [304], Large-scale Cryogenic Gravitational wave Telescope (LCGT) [216], and Fabry-Perot type DECIGO [217, 305]. It is noted that $h_{\text {char }}$ is the characteristic gravitational wave strain defined in [218]. The supernova is assumed to be located at the distance of $10 \mathrm{kpc}$. This figure is taken from [219].

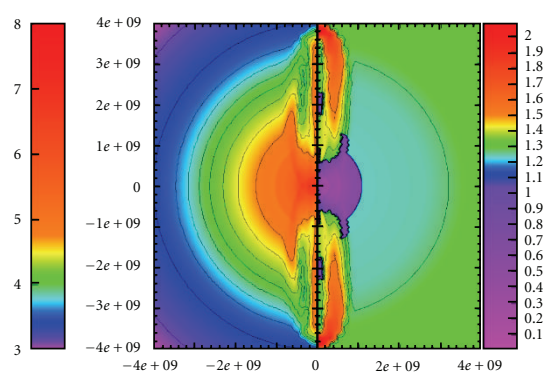

(a)
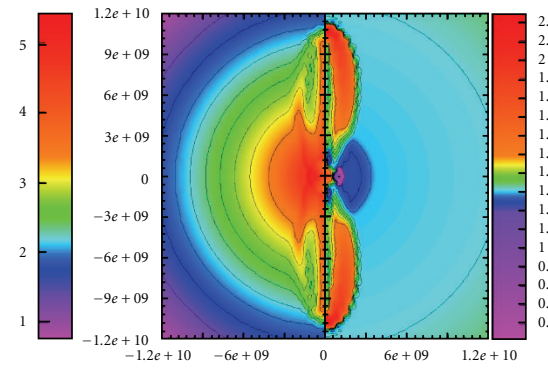

(b)

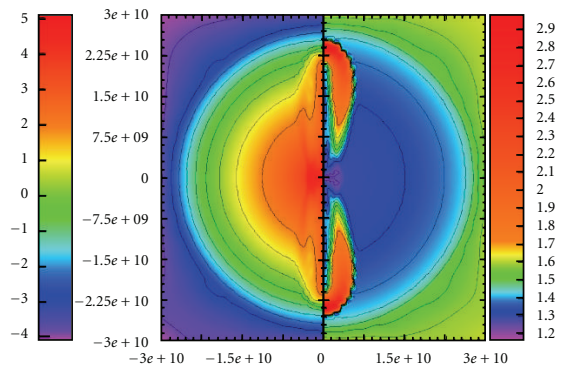

(c)

Figure 25: Snapshots showing MHD explosions of CCSNe at 0.8, 2.0, and $4.3 \mathrm{sec}$ after core bounce from left to right (e.g., [268]). In each figure, contour of the logarithmic density $\left(\mathrm{g} / \mathrm{cm}^{3}\right)(\mathrm{left})$ and entropy per baryon $\left(k_{B}\right)$ (right) are shown. The unit of the horizontal and the vertical axis is in $(\mathrm{cm})$. Note that the difference of the length scale for each panel and that the outermost radius of the progenitor is $\sim 3 \times 10^{10}(\mathrm{~cm})$.

a tomography, thus could provide a powerful test of the mixing angle and the mass hierarchy (e.g., [320, 323-325]).

It is rather recently that the importance of collective flavor oscillations was widely recognized. Neutrinos streaming out of the neutrino spheres are so dense that they provide a large matter effect for each other. The collective effect usually takes place between the neutrino sphere and the mentioned MSW region. Regardless of the inherent nonlinearity and the presence of multiangle effects, the final outcome for the emergent neutrino flux seems to be converging after a series of extensive study over the past years at least for the $1 \mathrm{D}$ models. Although the collective effects will not be significant to assist the neutrino-driven explosions (e.g., [326, 327], see however $[328,329])$, they are predicted to emerge as a distinct observable feature in their energy spectra (see [7477] for reviews of the rapidly growing research field and collective references therein).

An important lesson from SN1987A is that for explaining the duration of the events, there was no other energy-loss channel but for the ordinary neutrinos in the context of the Standard Model of particle physics (e.g., [6, 330]). The next SN event could provide an opportunity to study also a nontrivial property of neutrinos, such as the magnetic dipole moments. The resonant spin-flavor conversion has been also studied both analytically (e.g., [331-333] and references therein) and numerically (e.g., [334]), which can transform some of the prompt $\nu_{e}$ burst into $\bar{\nu}_{e}$ in highly magnetized supernova envelopes, leading to a huge $\bar{\nu}_{e}$ burst. 

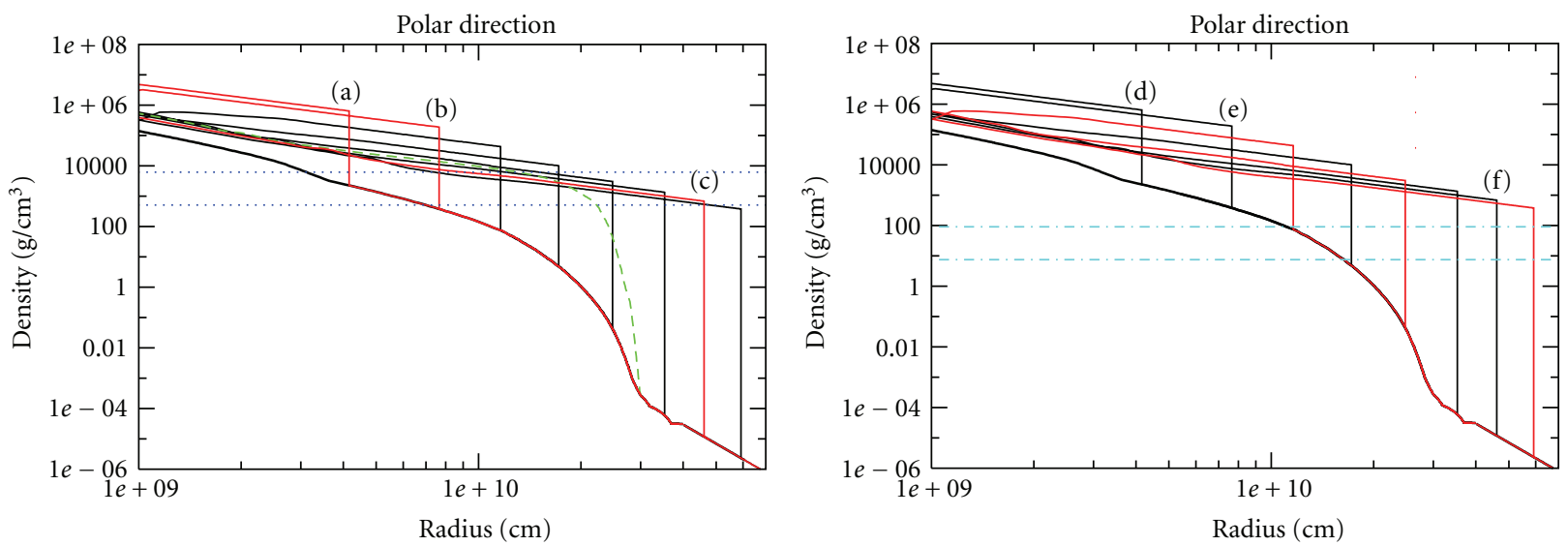

Figure 26: The density profile in the polar direction every $0.4 \mathrm{~s}$ as a function of radius. (a), (b) and (c) correspond to $0.4,0.8$ and $2.8 \mathrm{~s}$, respectively. The horizontal blue lines show approximately density of the H-resonance for different neutrino energies which are 5 (above) and $60 \mathrm{MeV}$ (below), respectively. The green line is an original density profile for $2.0 \mathrm{~s}$. Same as the left panel, but for $1.2 \mathrm{~s}$ (d), $2.0 \mathrm{~s}$ (e), and $3.2 \mathrm{~s}(\mathrm{f})$, respectively. Note that a sharpness of the shock along the polar direction is modified from the one in the MHD simulations (green line showing an original density profile for $2.0 \mathrm{~s}$ ), which is made to be inevitably blunt due to the employed numerical scheme using an artificial viscosity to capture shocks (taken from [306]).

The mentioned important ingredients related to the flavor conversions due to the MSW effects, collective effects, and the electromagnetic effects in the supernova environment have been studied often one by one in each study without putting all the effects together, possibly in order to highlight the new ingredient. In this sense, all the studies mentioned above should be regarded as complimentary towards the precise predictions of $\mathrm{SN}$ neutrinos.

Here it should be mentioned that most of those rich phenomenology of SN neutrinos have been based on 1D spherically symmetric simulations (e.g., $[75,310,312$, $315,319,320,335-338]$ and references therein). Apart from a simple parametrization to mimic anisotropy and Stochasticity of the shock (e.g., [339-341]), there have been not so many studies focusing how global asymmetry of the explosion dynamics obtained in recent supernova simulations (e.g., Sections 2 and 3 ) would affect the neutrino oscillations (see, however, $[79,342])$. This is mainly due to the lack of multi-D supernova models, which are very computationally expensive to continue the simulations till the shock waves propagate outward until they affect neutrino transformations. It is only recently that several studies along this line have been reported [133, 343], in which the collective or MSW effects are rendered to be treated in an approximate manner. By analyzing the $2 \mathrm{D}$ results of a $15 M_{\odot}$ model by Marek and Janka [125] who included one of the best available neutrino transfer approximations (e.g., Table 1), Lund et al. [343] pointed out that fast time variations caused by convection and SASI lead to significant modulations around a few hundred $\mathrm{Hz}$, which can be visible in IceCube or future megaton-class detectors for the galactic SN source. Based on the $2 \mathrm{D}$ results of $20 M_{\odot}$ models by Ott et al. [132] who reported the first 2D multiangle transport simulations, Brandt et al. [133] also obtained the similar results. From their rapidly rotating model, they also pointed out that a rapid rotation of precollapse $\mathrm{SN}$ cores imprints strong asymmetries in the neutrino flux [344] as well as in its light curves [133, 345].

In the following, we briefly summarize our findings [306] in which we studied exploratory the neutrino oscillations in the context of the MHD mechanism. As mentioned in Section 3, it is recently possible for special relativistic simulations to follow the dynamics of MHD explosions continuously, starting from the onset of gravitational collapse, through core-bounce, the magnetic shock-revival, till the shock-propagation to the stellar surface. Based on our models [268], we calculated numerically the neutrino flavor conversion in the highly nonspherical envelope through the MSW effect. The neutrino transport was simply treated by a leakage scheme (e.g., [268]). The emergent neutrino spectra was assumed to take a Fermi-Dirac type distribution function and the neutrino temperature was estimated to take the average matter temperature on the neutrino sphere. As explained in Section 3(a), the density profile along the polar and equatorial direction is very different due to the strong explosion anisotropy inherent to the MHD explosions. In the following, we pay attention to the anisotropic shock effect on the MSW effect. As will be discussed, we could observe a sharp dip in the neutrino event only seen from a polar direction, albeit depending on the mass hierarchy and the mixing angle of $\theta_{13}$. An advantage of the MHD models is that the shock revival can occur much faster after bounce compared to the other proposed mechanism. Therefore, the neutrino luminosity could remain higher than those in the other mechanisms, which could potentially enhance the detectability due to the early shock arrival to the resonance region. For simplicity, the effects of neutrino self-interactions are treated very phenomenologically and 


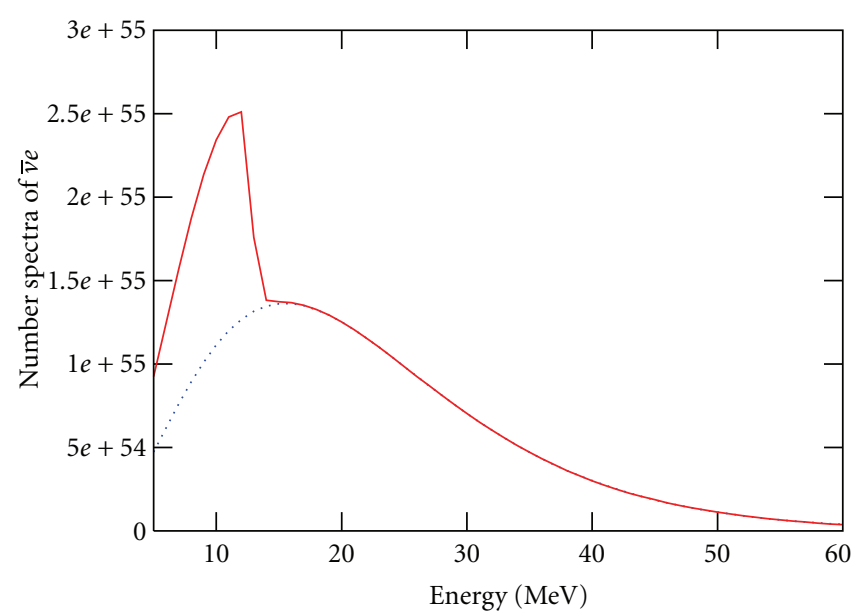

$\begin{array}{ll}- & \text { Polar direction } \\ \ldots . . & \text { Equatorial direction }\end{array}$

(a)

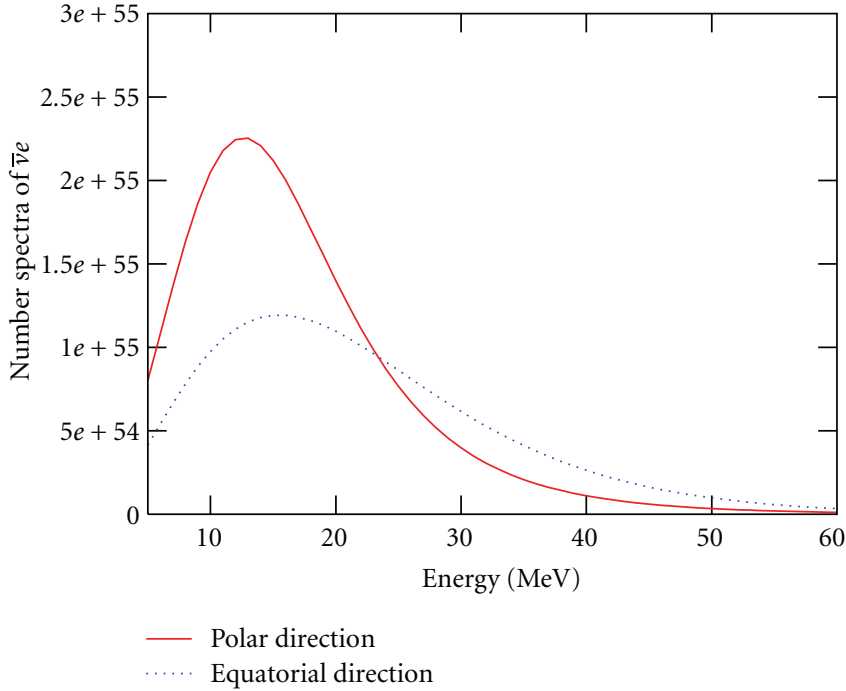

(b)

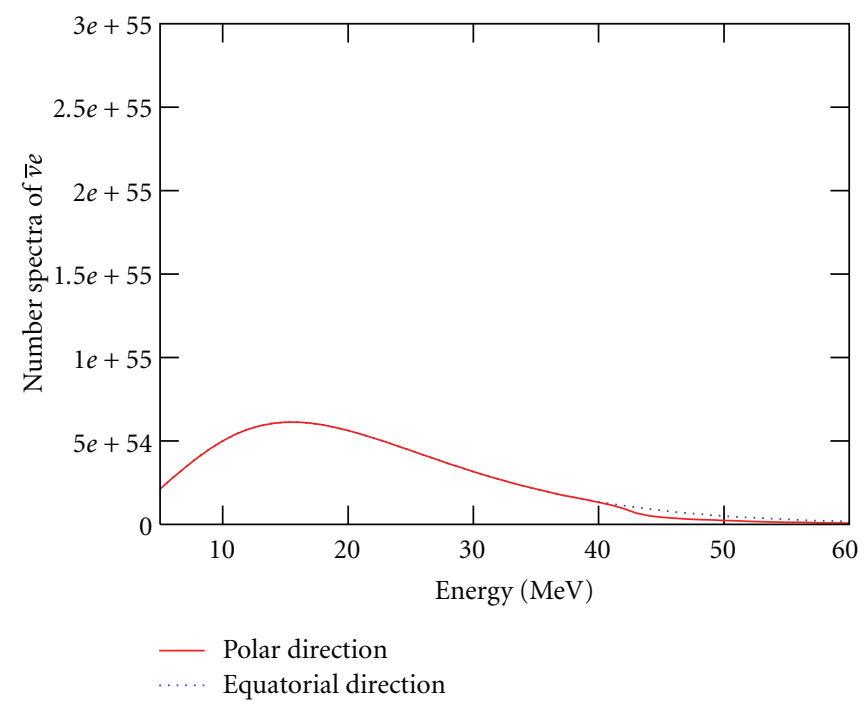

(c)

FIGURE 27: $\bar{v}_{e}$ spectra at the surface of the star in the case of (a), (b), and (c). We assume that the inverted mass hierarchy and $\sin ^{2} 2 \theta_{13}=10^{-3}$. The solid lines (red lines) and the dotted lines (blue lines) are the spectra of polar direction and equatorial direction, respectively. Note in this figure that the neutrino luminosity and spectra are modeled to be the same as those obtained in a $1 \mathrm{D}$ full-scale numerical simulation by the Lawrence Livermore group [91]. These figures are taken from [306].

the resonant spin-flavor is not considered. Even though far from comprehensive in this respect, we presented the first discussion how the magneto-driven explosion anisotropy has impacts on the emergent neutrino spectra and the resulting event number observed by the SK for a future Galactic supernova (e.g., [306]).

3.2.1. Possible Neutrino Signatures from MHD Explosions. One prominent feature of the MHD models is a high degree of the explosion asphericity. Figure 25 shows several snapshots featuring typical hydrodynamics of the model, from near core-bounce ( $0.8 \mathrm{~s}$, left), during the shock-propagation
( $2.0 \mathrm{~s}$, middle), till near the shock breakout from the star ( $4.3 \mathrm{~s}$, right), in which time is measured from the epoch of core bounce. It can be seen that the strong shock propagates outwards with time along the rotational axis. On the other hand, the density profile hardly changes in the equatorial direction, which is a generic feature of the MHD explosions.

As well known, the flavor conversion through the purematter MSW effect occurs in the resonance layer, where the density is

$$
\rho_{\text {res }} \sim 1.4 \times 10^{3} \mathrm{~g} / \mathrm{cm}^{3}\left(\frac{\Delta m^{2}}{10^{-3} \mathrm{eV}^{2}}\right)\left(\frac{10 \mathrm{MeV}}{E_{v}}\right)\left(\frac{0.5}{Y_{e}}\right) \cos 2 \theta,
$$




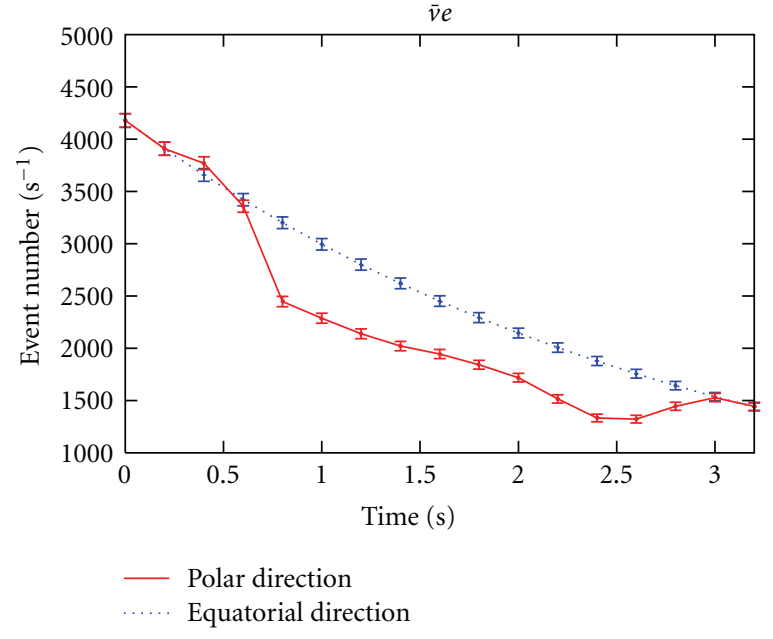

(a)

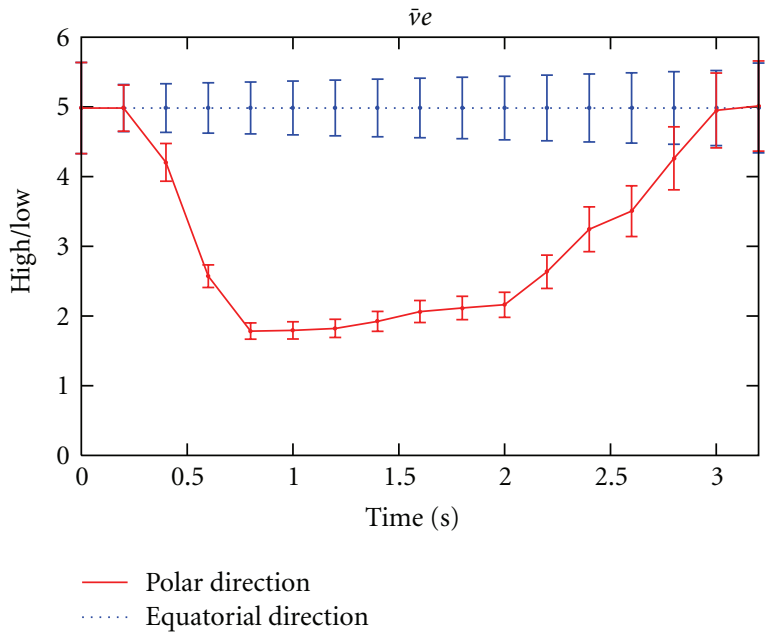

(b)

Figure 28: Same as Figure 27 but for the expected event number of $\bar{\nu}_{e}$ (left panel) in the SK as a function of the time. The solid line (red line) and the the dotted line (blue line) are for the polar and the equatorial directions, respectively. The supernova is assumed to be located at the distance of $10 \mathrm{kpc}$. The error bars represent the $1 \sigma$ statistical errors only. Right panel is for the ratio of high-to-low-energy events which can be a useful quantity to characterize the change (dip in the right panel) of neutrino signals by the shock effect (see [306] for details).

where $\Delta m^{2}$ is the mass squared difference, $E_{\nu}$ is the neutrino energy, $Y_{e}$ is the number of electrons per baryon, and $\theta$ is the mixing angle. Since the inner supernova core is too dense to allow MSW resonance conversion, we focus on two resonance points in the outer supernova envelope. One that occurs at higher density is called the $\mathrm{H}$-resonance, and the other, which occurs at lower density, is called the Lresonance. $\Delta m^{2}$ and $\theta$ correspond to $\Delta m_{13}^{2}$ and $\theta_{13}$ at the $\mathrm{H}$ resonance and to $\Delta m_{12}^{2}$ and $\theta_{12}$ at the L-resonance.

Figure 26 are evolutions of the density profiles in the polar direction every $0.4 \mathrm{~s}$ as a function of radius. Above and below horizontal lines show approximately density of the resonance for different neutrino energies which are 5 and $60 \mathrm{MeV}$, respectively. Blue lines (left panel) show the range of the density of the H-resonance, and sky-blue lines (right panel) show that of the L-resonance. Along the polar axis, the shock wave reaches to the H-resonance, $\sim O\left(10^{3}\right) \mathrm{g} / \mathrm{cm}^{3}$ at $\sim$ $0.5 \mathrm{~s}$, and the L-resonance, $\sim O(1) \mathrm{g} / \mathrm{cm}^{3}$ at $\sim 1.2 \mathrm{~s}$. It should be noted that those timescales are very early in comparison with the ones predicted in the neutrino-driven explosion models, typically $\sim 5 \mathrm{~s}$ and $\sim 15 \mathrm{~s}$ for the $\mathrm{H}$ - and L-resonances, respectively (e.g., $[320,325]$ ). This arises from the fact that the MHD explosion is triggered promptly after core bounce, which is in sharp contrast to the neutrino-driven delayed explosion models [320, 325]. The progenitor of the MHD models, possibly linked to long-duration gamma-ray bursts, is more compact due to a chemically homogeneous evolution $[274,275]$, which is also the reason for the early shock-arrival to the resonance regions.

Figure 27 is the energy spectra of $\bar{v}_{e}$ for (a), (b), and (c) in the case of inverted mass hierarchy. The solid lines (red lines) and the dotted lines (blue lines) are the spectra of the polar direction and the equatorial direction, respectively. At the panel (a), an enhancement of the low-energy side of the polar direction is seen, which is as a result of the supernova shock reaching to the H-resonance region. The survival probability of $\bar{v}_{e}$ can remain non-zero when the steep decline of the density at the shock front changes the resonance into non-adiabatic (see [306] for more details). As the shock propagates from the high density region to low energy region, the shock effect transits from low-energy side to high-energy side in spectra, because the density at the resonance point, $\rho_{\text {res }}$, is proportional to $E_{v}^{-1}$. In fact, the energy spectrum of the polar direction becomes softer in the high-energy side than for the equatorial direction as shown in Figure 27(b).

Figure 28 shows the time evolution of the event number in SK, where the solid line (red line) and the dotted line (blue line) are for the polar and the equatorial direction, respectively. The shock effect is clearly seen. The event number of the polar direction shows a steep decrease, marking the shock passage to the $\mathrm{H}$-resonance layer. The change of the event number by the shock passage is about $36 \%$ of the event number without the shock. Since the expected events are $\sim 2500$ at the sudden decrease (Figure 28), it seems to be quite possible to identify such a feature by the SK class detectors. Such a large number imprinting the shock effect, possibly up to two-orders-of magnitudes larger than the ones predicted in the neutrino-driven explosions (e.g., Figure 11 in $[320]$ ), is thanks to the mentioned early shock-arrival to the resonance layer, peculiar for the MHD explosions.

Finally we exploratory discuss possible impacts of the spectral swap between $\bar{v}_{e}$ and $v_{x}$, which is one of a probable outcome of the collective neutrino oscillations [346]. In our model, the original neutrino flux of $\bar{\nu}_{e}$ is larger than that of $v_{x}$ for the low-energy side, which is vice versa for the highenergy side. Due to the spectral swap, the above inequality sign reverses (see [306] for more detail). As a result, the 
TABle 2: A summary illustrating the candidate supernova mechanisms (vertical direction in the Table) and their expected features of the emergent multimessengers (horizontal direction). Regarding the photon messengers, possible signatures associated with nucleosynthesis are only partly presented (see Section 1 for a number of important findings regarding the light-curve and spectra modeling).

\begin{tabular}{|c|c|c|c|}
\hline Messenger & Gravitational waves & Neutrinos & $\begin{array}{c}\text { Photons } \\
\text { (nucleosynthesis) }\end{array}$ \\
\hline$\frac{\text { Canonical rotation }}{\gamma}$ & $\begin{array}{l}\text { Stochastic } \\
\text { (convection and SASI) }\end{array}$ & $\begin{array}{l}\text { Stochastic } \\
\text { (convection and SASI) }\end{array}$ & $\begin{array}{l}\nu \mathrm{p} \text { process } \\
\text { Anisotropic explosive } \\
\text { nucleosynthesis }\end{array}$ \\
\hline$\frac{1}{\text { Rapid rotation }}$ & $\begin{array}{c}\text { Stochastic (excess for equator } \\
\text { (spiral SASI modes) }\end{array}$ & Polar excess & $\nu \mathrm{p}$ process (?) \\
\hline Fails: black-hole forming & (bounce and $\mathrm{BH}$ formation) & Disappearing signals & No photon (?) \\
\hline MHD mechanism & $\begin{array}{l}\text { Burst and tail } \\
\text { (rapid rotation, non } \\
\text { axisymmetric instabilities, } \\
\text { and magnetic fields) }\end{array}$ & $\begin{array}{l}\text { (i) Polar excess (?) } \\
\text { (ii) Anisotropy in SK } \\
\text { events (MSW effect) } \\
\text { (iii) } \bar{v}_{e} \text { bursts (RSF) }\end{array}$ & $\begin{array}{l}\text { (i) } \nu \text { p process (?) } \\
\text { (ii) r-process cites (?) } \\
\text { (iii) Path to hypernovae (?) }\end{array}$ \\
\hline
\end{tabular}

event number in the polar direction is expected to increase by the influence of the shock. To draw a robust conclusion to the self-interaction effects, one needs to perform a more sophisticated analysis such as the multiangle approach (e.g., $[76]$ ), which is a major undertaking.

\section{Summary and Discussion}

The aim of writing this paper was to provide an overview of what we currently know about the explosion mechanism, neutrinos, gravitational waves, and explosive nucleosynthesis in CCSNe to bridge theory and observation through multimessenger astronomy. Not to inflate the volume, we had to limit ourselves to focus primarily on the findings based on our numerical studies, which are thus limited to GWs (Section 2.2) and explosive nucleosynthesis (Section 2.3) in the neutrino-heating mechanism (Section 2), and to GWs (Section 3.1) and neutrino signals (Section 3.2) in the MHD mechanism (Section 3). Apparently our current presentation is weak on the photon side mainly because there is a big gap to connect the outcomes obtained in the multiD neutrino-radiation-hydrodynamic simulations covering only the very centre of the star, to the light-curve and spectra modeling that requires multi-D photon-radiationhydrodynamic modeling (far) after the shock-breakout of the star. The gap is not only the matter of physical scale, but also the matter of numerical difficulty. Note that Table 2 is not intended to show an overview covering everything concerning the SN multimessengers, which is far beyond the scope of this review. We boldly present the incomplete figure here, hoping that the shortage might give momentum to theorists for fixing the problems and for gaining much more comprehensive multimessenger perspectives for the future.

In the neutrino-heating mechanism, stellar rotation holds a key importance to characterize the SN multimessengers. The whole story may be summarized as follows. If a precollapse iron core has a "canonical" rotation rate as predicted by recent stellar evolution calculations [126, 127], collapse-dynamics before bounce proceeds spherically and the postbounce structures interior to the PNS are essentially spherical. Typically later than $\sim 100 \mathrm{~ms}$ after bounce, the bounce shock turns to a standing accretion shock, and the activities of convective overturns and SASI become vigorous with time behind the standing shock. Since anisotropies of the neutrino flux and matter motions in the postbounce phase are governed by the nonlinear hydrodynamics, GWs emitted in this epoch also change stochastically with time (indicated by "Stochastic" in the table, e.g., Section 3.1 for more details). For detecting these signals for a Galactic CCSN event with a good signal-to-noise ratio, we need next generation detectors such as the advanced LIGO, LCGT, and the Fabry-Perot (FP-) type DECIGO. Neutrino signals at the nonlinear epoch also change stochastically with time, which is expected to be detected by currently running detector such as by IceCube and SK $[133,197,343]$ for the galactic event. It should be also mentioned that detailed neutrino transport including the most up-to-date interaction rates should be accurately implemented in the multi-D simulations to make a reliable prediction of the neutrino signals (e.g., $[312,313]$ ). When material behind the stalled shock successfully absorbs enough neutrino energy to be gravitationally unbound from 
the iron core, the $\nu \mathrm{p}$ process is expected to successfully explain some light proton-rich nuclei in the neutrinodriven wind phase [245-247]. When the revived supernova shock (successfully) attains the kinetic energy as energetic as $10^{51} \mathrm{erg}$, explosive nucleosynthesis that proceeds in the SASIaided low-mode explosions is expected to partly explain the solar abundance yields as well as the observed nonuniform morphology of the synthesized elements (e.g., Section 2.3.1).

One important notice here is that explosion energies obtained in the state-of-the-art multi-D simulations are typically underpowered by one or two orders-of-magnitudes to explain the canonical supernova kinetic energy $\left(\sim 10^{51} \mathrm{erg}\right.$, e.g., Table 1). Moreover, the softer EOS, such as of Lattimer and Swesty [347] (LS) EOS with an incompressibility at nuclear densities, $K$, of $180 \mathrm{MeV}$, is employed in those simulations (e.g., in multi-D simulations of the MPA, Oak Ridge+, and Tokyo+ groups in Table 1). On top of a striking evidence that favors a stiffer EOS based on the nuclear experimental data $(K=240 \pm 20 \mathrm{MeV},[348])$, the soft EOS may not account for the recently observed massive neutron star of $\sim 2 M_{\odot}$ [349] (see the maximum mass for the LS180 EOS in $[314,350])$. With a stiffer EOS, the explosion energy may be even lower as inferred from Marek and Janka [125] who did not obtain the neutrino-driven explosion for their model with $K=263 \mathrm{MeV}$ (on the other hand, they obtained 2D explosions for Shen EOS $(K=281 \mathrm{MeV})$ (Janka, private communication) $)$. What is then missing furthermore? We may get the answer by going to $3 \mathrm{D}$ simulations (Section 2.1) or by taking into account new ingredients, such as exotic physics in the core of the protoneutron star [123, 351], viscous heating by the magnetorotational instability (Section $3(b)$ ), or energy dissipation via Alfvén waves [352]. In addition to the 3D effects, GR is expected to help the onset of multi-D neutrinodriven explosions [169, 353] (e.g., [129] in 2D simulations but with detailed neutrino transport or [169] in 3D simulations but with a very approximate neutrino transport). At present, the most up-to-date neutrino transport code can treat the multienergy and multiangle transport in 2D [132] and even in 3D simulations [158] but only limited to the Newtonian simulations. Extrapolating a current growth rate of supercomputing power, the dream simulation (i.e., $6 \mathrm{D}$ simulations with full Boltzmann neutrino transport in full GR simulations) is likely to be practicable not in the distant future (in our lifetime!) presumably by using the exa-scale platforms.

In addition to these numerical advancements, the physical understanding of the supernova theory via analytical studies is also in a steady progress. What determines the saturation levels of SASI? A careful analysis on the parasitic instabilities has been reported to answer this question [354]. What determines mode couplings between small-scale convection eddies and large-scale SASI modes? To apply the theory of turbulence [355] should put a milestone to address this question. Besides the two representative EOSs of Lattimer-Swesty and Shen, new sets of EOSs have been recently reported [356-358]. Some of them will enable SN modellers to go beyond a single nucleus approximation, which is an important improvement for an accurate description of neutrino-nucleus interaction.

Rotation, albeit depending on its strength, can give a special direction (i.e., spin axis) in the supernova cores. As discussed in Section 2.2.2, stochastic nature of the GWs becomes weak in the presence of rotation as a result of the spiral SASI (It should be noted that the feature can be seen even in a slowly rotating progenitor that agrees with recent stellar evolution calculation [126, 127] (see Section 2.2.2 for more details)) (indicated by "excess for equator" in Table 2). Although it is not easy to detect these low-frequency GWs from anisotropic neutrino emission by currently running laser interferometers, a recently proposed spacebased interferometers (like FP-type DECIGO) would permit detection for a galactic event. Contributed by the neutrino GWs in the lower frequency domain, the total GW spectrum is expected to become rather flat over a broad frequency range below $\sim 1 \mathrm{kHz}$. This GW feature obtained in the context of the SASI-aided neutrino-driven mechanism is different from the one in the other candidate supernova mechanisms, such as the MHD mechanism (Section 3.1) and the acoustic mechanism [193], thus could provide an important probe into the explosion mechanism. Rapid rotation induces a polar excess of neutrino emission with a harder spectrum than on the equator $[133,344,345]$ (indicated by "polar excess" in the table). This should be also the case of the MHD mechanism, which deserves further investigation for a quantitative discussion (symbolized by Polar excess (?) in the table). These (rotation-induced) neutrino signatures, if observed, will carry an important information about the angular momentum profiles hidden deep inside massive stellar cores. Concerning the $\nu$ p process and explosive synthesis in rapidly rotating cores (as well as in the MHD explosions), there still remains a vast virgin territory for further investigation (symbolized by " $\nu$ p process (?)" in the table).

If the neutrino-heating mechanism (or some other mechanisms) fails to blow up massive stars, central PNSs collapse to BHs. Recent GR simulations by Ott et al. [199] show that the significant GW emission is associated at the moment of the $\mathrm{BH}$ formation, which can be a promising target of the advanced LIGO for a galactic source. As pointed out by Sumiyoshi et al. [359], the disappearing neutrino signals that mark the epoch of BH formations also can be a target of SK and IceCube [10]. When quarks and hyperons appear in the postbounce core, a neutrino burst produced by the sudden EOS softening and by the subsequent rebounce [123] is likely to be detected by IceCube for a galactic event [10]. The interval between core-bounce and the $\mathrm{BH}$ formation depends on the details of exotic physics in the super-dense PNS cores $[360,361]$. All of these observational signatures should provide an important probe into the so-called dense QCD region in the QCD phase diagram (e.g., [362] for recent review) to which lattice calculations are hardly accessible at present. Concerning photons, no optical outbursts are expected in the $\mathrm{BH}$-forming $\mathrm{SNe}$ (indicated by "no photon (?)" in Table 2), if not for rapid rotation and strong magnetic fields prior to core-collapse. 
If a precollapse core rotates enough rapidly (typically initial rotation period less than $4 \mathrm{~s}$ ) with strong magnetic fields (higher than $\sim 10^{11} \mathrm{G}$ ), the MHD mechanism can produce bipolar explosions along the rotational axis predominantly driven by the field wrapping processes (Section 3(a)). If the MRI can be sufficiently resolved in global simulations, the MRI would exponentially amplify the initial magnetic fields to a dynamically important strength within several rotational periods (Sections 3(b) and $3(\mathrm{c})$ ). The next-generation supercomputing resources are needed again to see the outcome. If such simulations would be executable, the MHD outflows will be produced even for more weakly magnetized cores than currently predicted. The GW signals in the MHD explosions are characterized by a burst-like bounce signal plus a secularly growing tail (Section 3.1, indicated by "burst and tail" in the table). Similar to the neutrino GWs in the neutrino-driven mechanism, a future detector (like FPtype DECIGO) is again necessary for detecting the lowfrequency tail component. As discussed in Section 3.2.1, the pole to equator anisotropy of the shock propagation in the MHD explosions affect the neutrino signals through the MSW effects. The anisotropic shock passage to the Hresonance regions leads to a sudden decrease in the SK events ( 2500 events for a galactic source), which is quite possible to identify by the SK-class detectors (indicated by "anisotropy in SK events" in Table 2). Since the MHD mechanism has such distinct signatures, a planned joint analysis of neutrino and GW data [363] would be potentially very powerful to tell the MHD mechanism from the other candidate mechanisms. If the resonant spin-flavor conversion (indicated as RSF in Table 2) occurs in the highly magnetized core, the neutronization burst of $v_{e}$ converts to that of $\bar{\nu}_{e}$, which is thus expected to be a probe into the magnetic moment of neutrinos (e.g., [334]). Finally the MHD explosions are considered to be a possible $r$ process cite [364, 365] (see collective references in [247] for other plausible $r$-process cites). This is because the mass ejection from the iron core occurs in much shorter timescales compared to the delayed neutrino-heating mechanism, so that the ejecta can stay neutron rich before it becomes proton-rich via neutrino capture reactions. Most of these possibilities regarding the MHD mechanism have been proposed so far in simulations with a crude treatment of neutrino transport, which is now rather easily reexamined by the state-of-the-art multi-D simulations (if the code is MHD).

Finally, what is about the story if the MHD mechanism fails? (indicated by "path to hypernovae in Table 2). The rapidly rotating PNS collapses into a $\mathrm{BH}$, probably leading to the formation of accretion disk around the $\mathrm{BH}$. Neutrinos emitted from the accretion disk heat matter in the polar funnel region to launch outflows or strong magnetic fields in the cores of the order of $10^{15} \mathrm{G}$ also play an active role both in driving the magneto-driven jets and in extracting a significant amount of energy from the $\mathrm{BH}$ (e.g., [366-368] and see references therein). This picture, often referred as collapsar (e.g., [298, 299]), has been the working hypothesis as a central engine of long-duration GRBs for these 10 years (see references in [367-371] for other candidate mechanism including magnetar models). However, it is still controversial whether the generation of the relativistic outflows predominantly proceeds via the neutrino-heating mechanism or the MHD mechanism. In contrast to a number of findings illustrated in Table 2, much little things are known about the $\mathrm{BH}$-forming supernovae and also about the collapsar. This is mainly because the requirement for making a realistic numerical modeling is very computationally expensive, which (at least) necessitates the multi-D MHD simulations not only with GR for handling the $\mathrm{BH}$ formation (thus full GR), but also with the multiangle neutrino transfer for treating highly anisotropic neutrino radiation from the accretion disk [372]. To get a unified picture of massive stellar death, we need to draw a schematic picture (like Table 2) also in the case of the $\mathrm{BH}$-forming supernovae. We anticipate that our long-lasting focus (and experience) on the explosion dynamics of canonical CCSNe will be readily applicable to clarifying the origin of the gigantic explosion energy of hypernovae and also unraveling (ultimately) the central engine of long-duration GRBs. This should provide yet another grand challenge in computational astrophysics in the next decades.

As repeatedly mentioned so far, all the numerical results in this paper should be tested by the next-generation calculations by which more sophistication is made not only in the treatment of multi-D radiation transport (both of neutrino and photon), but also in multi-D hydrodynamics including stellar rotation and magnetic fields in full GR. From an optimistic point of view, our understanding on every issue raised in this paper can progress in a step-bystep manner at the same pace as our available computational resources will be growing bigger and bigger from now on. Since 2009, several neutrino detectors form the Supernova Early Warning Systems (SNEWS) to broadcast the alert to astronomers to let them know the arrival of neutrinos [82]. Currently, Super-Kamiokande, LVD, Borexino, and IceCube contributes to the SNEWS, with a number of other neutrino and GW detectors planning to join in the near future. This is a very encouraging news towards the highprecision multimessenger astronomy. The interplay between the detailed numerical modeling, the advancing supercomputing resources, and the multimessenger astronomy, will remain a central issue for advancing our understanding of the theory of massive stellar core-collapse for the future. A documentary film recording our endeavors to make practicable the "multimessenger astronomy of CCSNe" seems not to show " $f i n$ " immediately and is becoming even longer as far as the three components evolve with time. To raise the edifice, it is becoming increasingly important to bring forward a worldwide, multidisciplinary collaboration among different research groups. We believe that such an approach would provide the shortest cut to get a deeper understanding of a number of unsettled and exciting issues that we were only able to touch in this paper. 


\section{Acknowledgments}

K. Kotake is thankful to K. Sato for continuing encouragements. He also wishes to thank his collaborators, S. Yamada, M. Liebendörfer, N. Ohnishi, K. Sumiyoshi, K. Nakamura, T. Kuroda, M. Hashimoto, S. Harikae, N. Yasutake, N. Nishimura, K. Shaku, and H. Suzuki. The authors would like to acknowledge helpful exchanges and stimulating discussions with E. Müller, T. Foglizzo, H.-T. Janka, C. D. Ott, C. Fryer, J. Novak, S. Ando, P. Cerdá-Durán, M. Obergaulinger, J. Murphy, R. Fernández, E. O’Connor, M. Shibata, T. Font, and J. M. Ibañez. They are also grateful to our anonymous referees who gave us a number of valuable comments to enhance the quality of this paper. Numerical computations were carried out in part on XT4 and general common use computer system at the center for Computational Astrophysics, CfCA, the National Astronomical Observatory of Japan. This study was supported in part by the Grantsin-Aid for the Scientific Research from the Ministry of Education, Science and Culture of Japan (nos. 19540309, 20740150, 23540323, 23340069, and 24244036) and by HPCI Strategic Program of Japanese MEXT.

\section{References}

[1] S. Ando, J. F. Beacom, and H. Yüksel, "Detection of neutrinos from supernovae in nearby galaxies," Physical Review Letters, vol. 95, no. 17, 4171101 pages, 2005.

[2] K. Hirata, T. Kajita, M. Koshiba, M. Nakahata, and Y. Oyama, "Observation of a neutrino burst from the supernova SN1987A," Physical Review Letters, vol. 58, no. 14, pp. 14901493, 1987.

[3] R. M. Bionta, G. Blewitt, C. B. Bratton et al., "Observation of a neutrino burst in coincidence with supernova 1987A in the large magellanic cloud," Physical Review Letters, vol. 58, no. 14, pp. 1494-1496, 1987.

[4] K. Sato and H. Suzuki, "Analysis of neutrino burst from the supernova 1987A in the large magellanic cloud," Physical Review Letters, vol. 58, no. 25, pp. 2722-2725, 1987.

[5] G. Raffelt, "Neutrinos and the stars," in press, http://arxiv. org/abs/1201.1637.

[6] G. G. Raffelt, "Physics with supernovae," Nuclear Physics B, vol. 110, pp. 254-267, 2002.

[7] Y. Totsuka, "Neutrino astronomy," Reports on Progress in Physics, vol. 55, no. 3, article 377, 1992.

[8] K. Hultqvist and IceCube collaboration, "IceCube: physics, status, and future," Nuclear Instruments and Methods in Physics Research A, vol. 626-627, supplement, pp. S6-S12, 2011.

[9] A. Suzuki, "Present status of KamLand," Nuclear Physics B, vol. 77, no. 1-3, pp. 171-176, 1999.

[10] IceCube Collaboration, R. Abbasi, Y. Abdou et al., "IceCube sensitivity for low-energy neutrinos from nearby supernovae," in press, http://arxiv.org/abs/1108.0171.

[11] B. Abbott, R. Abbott, R. Adhikari et al., "Upper limits from the LIGO and TAMA detectors on the rate of gravitationalwave bursts," Physical Review D, vol. 72, no. 12, Article ID 122004, 16 pages, 2005.

[12] M. Ando and The TAMA Collaboration, "Current status of the TAMA300 gravitational-wave detector," Classical and Quantum Gravity, vol. 22, no. 18, pp. S881-S889, 2005.
[13] J. Hough, S. Rowan, and B. S. Sathyaprakash, "The search for gravitational waves," Journal of Physics B, vol. 38, no. 9, article S497, 2005.

[14] M. van der Sluys, "Gravitational waves from compact binaries," . In press, http://arxiv.org/abs/1108.1307.

[15] G. M. Harry and The LIGO Scientific Collaboration, "Advanced LIGO: the next generation of gravitational wave detectors," Classical and Quantum Gravity, vol. 27, no. 8, Article ID 084006, 2010.

[16] S. A. Balbus and J. F. Hawley, "Instability, turbulence, and enhanced transport in accretion disks," Reviews of Modern Physics, vol. 70, no. 1, pp. 1-53, 1998.

[17] B. S. Sathyaprakash and B. F. Schutz, "Physics, astrophysics and cosmology with gravitational waves," Living Reviews in Relativity, vol. 12, article 2, 2009.

[18] J. Faber, "Status of neutron star-black hole and binary neutron star simulations," Classical and Quantum Gravity, vol. 26, no. 11, Article ID 114004, 2009.

[19] M. D. Duez, "Numerical relativity confronts compact neutron star binaries: a review and status report," Classical and Quantum Gravity, vol. 27, no. 11, Article ID 114002, 2010.

[20] N. Andersson, V. Ferrari, D. I. Jones et al., "Gravitational waves from neutron stars: promises and challenges," General Relativity and Gravitation, vol. 43, no. 2, pp. 409-436, 2011.

[21] C. J. Horowitz, "Multi-messenger observations of neutron rich matter," International Journal of Modern Physics E, vol. 20, pp. 2077-2100, 2011.

[22] K. Kotake, K. Sato, and K. Takahashi, "Explosion mechanism, neutrino burst and gravitational wave in core-collapse supernovae," Reports on Progress in Physics, vol. 69, no. 4, article 971, 2006.

[23] C. D. Ott, "The gravitational-wave signature of core-collapse supernovae," Classical and Quantum Gravity, vol. 26, no. 6, Article ID 063001, 2009.

[24] C. L. Fryer and K. C. B. New, "Gravitational waves from gravitational collapse," Living Reviews in Relativity, vol. 14, no. $1,2011$.

[25] S. L. Shapiro and S. A. Teukolsky, Black Holes, White Dwarfs and Neutron Stars: The Physics of Compact Objects, The National Science Foundation, Wiley-Interscience, New York, NY, USA, 1983.

[26] L. Wang and J. C. Wheeler, "Spectropolarimetry of supernovae," Annual Review of Astronomy and Astrophysics, vol. 46, no. 1, pp. 433-474, 2008.

[27] S. E. Woosley, “SN 1987A-after the peak," The Astrophysical Journal, vol. 330, pp. 218-253, 1988.

[28] S. Blinnikov, P. Lundqvist, O. Bartunov, K. Nomoto, and K. Iwamoto, "Radiation hydrodynamics of SN 1987A. I. Global analysis of the light curve for the first 4 months," The Astrophysical Journal, vol. 532, no. 2, pp. 1132-1149, 2000.

[29] V. P. Utrobin, "The light curve of supernova 1987A: the structure of the presupernova and radioactive nickel mixing," Astronomy Letters, vol. 30, no. 5, pp. 293-308, 2004.

[30] S. E. Woosley and A. Heger, "Nucleosynthesis and remnants in massive stars of solar metallicity," Physics Reports, vol. 442, no. 1-, pp. 269-283, 2007.

[31] D. Arnett, B. Fryxell, and E. Mueller, "Instabilities and nonradial motion in SN 1987A," The Astrophysical Journal, vol. 341, pp. L63-L66, 1989.

[32] E. Müller, B. Fryxell, and D. Arnett, "Instability and clumping in SN 1987A," Astronomy and Astrophysics, vol. 251, no. 2, pp. 505-514, 1991. 
[33] K. Kifonidis, T. Plewa, H. T. Janka, and E. Müller, "Nonspherical core collapse supernovae I. Neutrino-driven convection, Rayleigh-Taylor instabilities, and the formation and propagation of metal clumps," Astronomy and Astrophysics, vol. 408, no. 2, pp. 621-649, 2003.

[34] N. J. Hammer, H. T. Janka, and E. Müller, "Threedimensional simulations of mixing instabilities in supernova explosions," The Astrophysical Journal, vol. 714, no. 2, pp. 1371-1385, 2010.

[35] S. Nagataki, M. A. Hashimoto, K. Sato, and S. Yamada, "Explosive nucleosynthesis in axisymmetrically deformed type II supernovae," The Astrophysical Journal, vol. 486, no. 2, pp. 1026-1035, 1997.

[36] S. Nagataki, "Effects of jetlike explosion in SN 1987A," Astrophysical Journal Supplement Series, vol. 127, no. 1, pp. 141-157, 2000.

[37] V. P. Utrobin and N. N. Chugai, "Supernova 2000cb: highenergy version of SN 1987A," Astronomy and Astrophysics, vol. 532, article A100, 6 pages, 2011.

[38] L. Wang, J. C. Wheeler, P. Hoflich et al., "The axisymmetric ejecta of supernova 1987A," The Astrophysical Journal, vol. 579, no. 2, article 671, 2002.

[39] K. Kjær, B. Leibundgut, C. Fransson, A. Jerkstrand, and J. Spyromilio, "The 3-D structure of SN 1987A's inner ejecta," Astronomy and Astrophysics, vol. 517, article A51, 10 pages, 2010.

[40] L. Wang, D. A. Howell, P. Hoflich, and J. C. Wheeler, "Bipolar Supernova Explosions," The Astrophysical Journal, vol. 550, article 1030, 2001.

[41] D. C. Leonard, A. V. Filippenko, M. Ganeshalingam et al., "A non-spherical core in the explosion of supernova SN 2004dj," Nature, vol. 440, no. 7083, pp. 505-507, 2006.

[42] D. C. Leonard, A. V. Filippenko, M. Ganeshalingam et al., "A non-spherical core in the explosion of supernova SN 2004dj," Nature, vol. 440, no. 7083, pp. 505-507, 2006.

[43] K. Kifonidis, T. Plewa, L. Scheck, H. T. Janka, and E. Müller, "Non-spherical core collapse supernovae," Astronomy and Astrophysics, vol. 453, no. 2, pp. 661-678, 2006.

[44] L. Scheck, K. Kifonidis, H. T. Janka, and E. Müller, "Multidimensional supernova simulations with approximative neutrino transport," Astronomy and Astrophysics, vol. 457, no. 3, pp. 963-986, 2006.

[45] A. Burrows, E. Livne, L. Dessart, C. D. Ott, and J. Murphy, "Features of the acoustic mechanism of core-collapse supernova explosions," The Astrophysical Journal, vol. 655, no. 1 I, pp. 416-433, 2007.

[46] A. Burrows, L. Dessart, E. Livne, C. D. Ott, and J. Murphy, "Simulations of magnetically driven supernova and hypernova explosions in the context of rapid rotation," The Astrophysical Journal, vol. 664, no. 1, article 416, 2007.

[47] L. Dessart and D. J. Hillier, "Supernova radiative-transfer modelling: a new approach using non-local thermodynamic equilibrium and full time dependence," Monthly Notices of the Royal Astronomical Society, vol. 405, no. 4, pp. 2141-2160, 2010.

[48] L. Dessart and D. J. Hillier, "Time-dependent effects in photospheric-phase type II supernova spectra," Monthly Notices of the Royal Astronomical Society, vol. 383, no. 1, pp. 57-74, 2008.

[49] L. Dessart and D. J. Hillier, "Quantitative spectroscopy of photospheric-phase type II supernovae," Astronomy and Astrophysics, vol. 437, no. 2, pp. 667-685, 2005.
[50] D. Kasen, R. C. Thomas, and P. Nugent, "Time-dependent Monte Carlo radiative transfer calculations for threedimensional supernova spectra, light curves, and polarization," The Astrophysical Journal, vol. 651, no. 1, article 366, 2006.

[51] D. Kasen and S. E. Woosley, “Type II supernovae: model light curves and standard candle relationships," The Astrophysical Journal, vol. 703, no. 2, pp. 2205-2216, 2009.

[52] L. Dessart and D. J. Hillier, "Synthetic line and continuum linear-polarization signatures of axisymmetric type II supernova ejecta," Monthly Notices of the Royal Astronomical Society, vol. 415, no. 4, pp. 3497-3519, 2011.

[53] D. C. Leonard, A. V. Filippenko, M. Ganeshalingam et al., "A non-spherical core in the explosion of supernova SN 2004dj," Nature, vol. 440, no. 7083, pp. 505-507, 2006.

[54] R. Chornock, A. V. Filippenko, W. Li, and J. M. Silverman, "LArge late-time asphericities in three type IIP supernovae," The Astrophysical Journal Letters, vol. 713, no. 2, pp. 13631375, 2010.

[55] R. A. Fesen, M. C. Hammell, J. Morse et al., "Discovery of outlying high-velocity oxygen-rich ejecta in Cassiopeia A," The Astrophysical Journal, vol. 636, pp. 859-872, 2006.

[56] R. A. Fesen, J. A. Zastrow, M. C. Hammell, J. M. Shull, and D. W. Silvia, "Ejecta knot flickering, mass ablation, and fragmentation in Cassiopeia A," The Astrophysical Journal, vol. 736, article 109, 2011.

[57] B. Aschenbach, R. Egger, and J. Trümper, "Discovery of explosion fragments outside the Vela supernova remnant shock-wave boundary," Nature, vol. 373, no. 6515, pp. 587590, 1995.

[58] J. P. Hughes, C. E. Rakowski, D. N. Burrows, and P. O. Slane, "Nucleosynthesis and mixing in Cassiopeia A," The Astrophysical Journal, vol. 528, no. 2, pp. L109-L113, 2000.

[59] M. Miceli, F. Bocchino, and F. Reale, "Physical and chemical inhomogeneities inside the Vela SNR shell. Indications of ejecta shrapnels," The Astrophysical Journal, vol. 676, no. 2, article 1064, 2008.

[60] S. J. Smartt, "Progenitors of core-collapse supernovae," Annual Review of Astronomy and Astrophysics, vol. 47, pp. 63106, 2009.

[61] J. W. Murphy, Z. G. Jennings, B. Williams, J. J. Dalcanton, and A. E. Dolphin, "The progenitor mass of SN 2011dh from stellar population analysis," The Astrophysical Journal, vol. 742, article L4, 2011.

[62] S. E. Woosley and J. S. Bloom, "The supernova gammaray burst connection," Annual Review of Astronomy and Astrophysics, vol. 44, no. 1, pp. 507-556, 2006.

[63] M. Modjaz, "Stellar forensics with the supernova-GRB connection-Ludwig Biermann award lecture 2010," Astronomische Nachrichten, vol. 332, no. 5, pp. 434-447, 2011.

[64] P. Podsiadlowski, N. Langer, A. J. T. Poelarends, S. Rappaport, A. Heger, and E. Pfahl, "The effects of binary evolution on the dynamics of core collapse and neutron star kicks," The Astrophysical Journal, vol. 612, no. 2, pp. 1044-1051, 2004.

[65] P. Podsiadlowski, N. Ivanova, S. Justham, and S. Rappaport, "Explosive common-envelope ejection: implications for gamma-ray bursts and low-mass black-hole binaries," Monthly Notices of the Royal Astronomical Society, vol. 406, article 840, 2010.

[66] C. L. Fryer, P. A. Mazzali, J. Prochaska et al., "Constraints on type Ib/c supernovae and gamma-ray burst progenitors," 
Publications of the Astronomical Society of the Pacific, vol. 119, no. 861, article 1211, 2007.

[67] N. Smith, W. Li, A. V. Filippenko, and R. Chornock, "Observed fractions of core-collapse supernova types and initial masses of their single and binary progenitor stars," Monthly Notices of the Royal Astronomical Society, vol. 412, pp. 1522-1538, 2011.

[68] S. C. Yoon and N. Langer, "Evolution of rapidly rotating metal-poor massive stars towards gamma-ray bursts," Astronomy and Astrophysics, vol. 443, no. 2, pp. 643-648, 2005.

[69] S. C. Yoon, S. E. Woosley, and N. Langer, "Type Ib/c supernovae in binary systems. I. Evolution and properties of the progenitor stars," The Astrophysical Journal, vol. 725, no. 1, pp. 940-954, 2010.

[70] K. Nomoto, N. Tominaga, H. Umeda, C. Kobayashi, and K. Maeda, "Nucleosynthesis yields of core-collapse supernovae and hypernovae, and galactic chemical evolution," Nuclear Physics A, vol. 777, pp. 424-458, 2006.

[71] L. Dessart, D. J. Hillier, E. Livne et al., "Core-collapse explosions of Wolf-Rayet stars and the connection to type IIb/Ib/Ic supernovae," Monthly Notices of the Royal Astronomical Society, vol. 414, no. 4, pp. 2985-3005, 2011.

[72] W. D. Arnett and C. Meakin, "Towards realistic progenitors of core-collapse supernovae," The Astrophysical Journal Letters, vol. 733, no. 2, article 78, 2011.

[73] C. A. Meakin and D. Arnett, "Turbulent convection in stellar interiors. I. Hydrodynamic simulation," The Astrophysical Journal, vol. 667, pp. 448-475, 2007.

[74] G. G. Raffelt and A. Y. Smirnov, "Self-induced spectral splits in supernova neutrino fluxes," Physical Review D, vol. 76, no. 8, Article ID 081301, 5 pages, 2007.

[75] H. Duan, G. M. Fuller, J. Carlson, and Y. Z. Qian, "Flavor evolution of the neutronization neutrino burst from an $\mathrm{O}-$ $\mathrm{Ne}-\mathrm{Mg}$ core-collapse supernova," Physical Review Letters, vol. 100, no. 2, Article ID 021101, 4 pages, 2008.

[76] H. Duan, G. M. Fuller, and Y. Qian, "Collective neutrino oscillations," Annual Review of Nuclear and Particle Science, vol. 60, pp. 569-594, 2010.

[77] B. Dasgupta, "Physics and astrophysics opportunities with supernova neutrinos," ArXiv e-prints.

[78] S. P. Mikheev and A. I. Smirnov, "Resonant amplification of $v$ oscillations in matter and solar-neutrino spectroscopy," Nuovo Cimento C, vol. 9, no. 1, pp. 17-26, 1986.

[79] H. Duan and J. P. Kneller, "Neutrino flavour transformation in supernovae," Journal of Physics G, vol. 36, no. 11, Article ID 113201, 2009.

[80] H. T. Janka, K. Langanke, A. Marek, G. Martínez-Pinedo, and B. Müller, "Theory of core-collapse supernovae," Physics Reports, vol. 442, no. 1-6, pp. 38-74, 2007.

[81] A. Burrows, L. Dessart, C. D. Ott, and E. Livne, "Multidimensional explorations in supernova theory," Physics Reports, vol. 442, p. 23, 2007.

[82] P. Antonioli, R. T. Fienberg, F. Fleurot et al., "SNEWS: the supernova early warning system," New Journal of Physics, vol. 6, article 114, 2004.

[83] F. Patat, D. Baade, P. Hoflich, J. R. Maund, L. Wang, and J. C. Wheeler, "VLT spectropolarimetry of the fast expanding type Ia SN 2006X," Astronomy and Astrophysics, vol. 508, no. 1, pp. 229-246, 2009.

[84] M. Tanaka, K. S. Kawabata, K. Maeda et al., "Spectropolarimetry of the unique type Ib supernova 2005bf: larger asymmetry revealed by later-phase data," The Astrophysical Journal, vol. 699, no. 2, article 1119, 2009.

[85] F. K. Thielemann, R. Hirschi, M. Liebendörfer, and R. Diehl, "Massive Stars and Their Supernovae," in Lecture Notes in Physics, R. Diehl, D. H. Hartmann, and N. Prantzos, Eds., vol. 182, pp. 153-232, Springer, Berlin, Germany, 2011.

[86] E. Chassande-Mottin, M. Hendry, P. J. Sutton, and S. Márka, "Multimessenger astronomy with the Einstein telescope," General Relativity and Gravitation, vol. 43, no. 2, pp. 437464, 2011.

[87] S. Márka, The LIGO Scientific Collaboration, and The Virgo Collaboration, "The path to the enhanced and advanced LIGO gravitational-wave detectors," Classical and Quantum Gravity, vol. 26, no. 11, Article ID 114013, 2009.

[88] T. Pradier and Antares Collaboration, "The Antares neutrino telescope and multi-messenger astronomy," Classical and Quantum Gravity, vol. 27, no. 19, Article ID 194004, 2010.

[89] Y. Aso, Z. Márka, C. Finley, J. Dwyer, K. Kotake, and S. Márka, "Search method for coincident events from LIGO and IceCube detectors," Classical and Quantum Gravity, vol. 25, no. 11, Article ID 114039, 2008.

[90] S. A. Colgate and R. H. White, "The hydrodynamic behavior of supernovae explosions," The Astrophysical Journal, vol. 143, article 626, 1966.

[91] J. R. Wilson, Numerical Astrophysics, 1985.

[92] H. A. Bethe and J. R. Wilson, "Revival of a stalled supernova shock by neutrino heating," The Astrophysical Journal, vol. 295, pp. 14-23, 1985.

[93] H. A. Bethe, "Supernova mechanisms," Reviews of Modern Physics, vol. 62, no. 4, pp. 801-866, 1990.

[94] M. Rampp and H. T. Janka, "Spherically symmetric simulation with Boltzmann neutrino transport of core collapse and postbounce evolution of a $15 \mathrm{M}$ star," The Astrophysical Journal Letters, vol. 539, no. 1, article L33, 2000.

[95] M. Liebendörfer, A. Mezzacappa, and F. K. Thielemann, "Conservative general relativistic radiation hydrodynamics in spherical symmetry and comoving coordinates," Physical Review D, vol. 63, no. 10, Article ID 104003, 14 pages, 2001.

[96] T. A. Thompson, A. Burrows, and P. A. Pinto, "Shock breakout in core-collapse supernovae and its neutrino signature," The Astrophysical Journal, vol. 592, no. 1, article 434, 2003.

[97] K. Sumiyoshi, S. Yamada, H. Suzuki, H. Shen, S. Chiba, and H. Toki, "Postbounce evolution of core-collapse supernovae: long-term effects of the equation of state," The Astrophysical Journal, vol. 629, no. 2, article 922, 2005.

[98] M. Herant, W. Benz, W. R. Hix, C. L. Fryer, and S. A. Colgate, "Inside the supernova: a powerful convective engine," The Astrophysical Journal, vol. 435, no. 1, pp. 339-361, 1994.

[99] A. Burrows, J. Hayes, and B. A. Fryxell, "On the nature of core-collapse supernova explosions," The Astrophysical Journal Letters, vol. 450, no. 2, pp. 830-850, 1995.

[100] H. T. Janka and E. Müller, "Neutrino heating, convection, and the mechanism of type-II supernova explosions," Astronomy and Astrophysics, vol. 306, article 167, 1996.

[101] C. L. Fryer, D. E. Holz, and S. A. Hughes, "Gravitational wave emission from core collapse of massive stars," The Astrophysical Journal, vol. 565, no. 1, pp. 430-446, 2002.

[102] C. L. Fryer, "Neutron star kicks from asymmetric collapse," The Astrophysical Journal Letters, vol. 601, no. 2, article L175, 2004.

[103] J. M. Blondin, A. Mezzacappa, and C. Demarino, "Stability of standing accretion shocks, with an eye toward core-collapse 
supernovae," The Astrophysical Journal Letters, vol. 584, no. 2, pp. 971-980, 2003.

[104] L. Scheck, T. Plewa, H. T. Janka, K. Kifonidis, and E. Müller, "Pulsar recoil by large-scale anisotropies in supernova explosions," Physical Review Letters, vol. 92, no. 1, Article ID 011103, 4 pages, 2004.

[105] N. Ohnishi, K. Kotake, and S. Yamada, "Numerical analysis of standing accretion shock instability with neutrino heating in supernova cores," The Astrophysical Journal, vol. 641, pp. 1018-1028, 2006.

[106] N. Ohnishi, K. Kotake, and S. Yamada, "Inelastic neutrinohelium scatterings and standing accretion shock instability in core-collapse supernovae," The Astrophysical Journal, vol. 667, pp. 375-381, 2007.

[107] T. Foglizzo, L. Scheck, and H. T. Janka, "Neutrino-driven convection versus advection in core-collapse supernovae," The Astrophysical Journal, vol. 625, pp. 1436-1450, 2006.

[108] J. W. Murphy and A. Burrows, "Criteria for core-collapse supernova explosions by the neutrino mechanism," The Astrophysical Journal, vol. 688, no. 2, pp. 1159-1175, 2008.

[109] R. Fernández and C. Thompson, "Stability of a spherical accretion shock with nuclear dissociation," The Astrophysical Journal, vol. 697, no. 2, pp. 1827-1841, 2009.

[110] R. Fernández and C. Thompson, "Dynamics of a spherical accretion shock with neutrino heating and alpha-particle recombination," The Astrophysical Journal, vol. 703, no. 2, pp. 1464-1464, 2009.

[111] W. Iwakami, K. Kotake, N. Ohnishi, S. Yamada, and K. Sawada, "Three-dimensional simulations of standing accretion shock instability in core-collapse supernovae," The Astrophysical Journal, vol. 678, no. 2, article 1207, 2008.

[112] W. Iwakami, K. Kotake, N. Ohnishi, S. Yamada, and K. Sawada, "Effects of rotation on standing accretion shock instability in non-linear phase for core-collapse supernovae," The Astrophysical Journal, vol. 700, no. 1, article 232, 2009.

[113] R. Fernández, "The spiral modes of the standing accretion shock instability," The Astrophysical Journal, vol. 725, no. 2, pp. 1563-1580, 2010.

[114] H. T. Janka, "Conditions for shock revival by neutrino heating in core-collapse supernovae," Astronomy and Astrophysics, vol. 368, pp. 527-560, 2001.

[115] A. Wongwathanarat, N. J. Hammer, and E. Müller, "An axisfree overset grid in spherical polar coordinates for simulating 3D self-gravitating flows," Astronomy and Astrophysics, vol. 514, article A48, 14 pages, 2010.

[116] J. Nordhaus, A. Burrows, A. Almgren, and J. Bell, "Dimension as a key to the neutrino mechanism of core-collapse supernova explosions," The Astrophysical Journal, vol. 720, no. 1 , article $694,2010$.

[117] K. Nomoto and M. Mashimoto, "Presupernova evolution of massive stars," Physics Reports, vol. 163, no. 1-3, pp. 13-36, 1988.

[118] S. E. Woosley, A. Heger, and T. A. Weaver, "The evolution and explosion of massive stars," Reviews of Modern Physics, vol. 74, no. 4, pp. 1015-1071, 2002.

[119] S. E. Woosley and T. A. Weaver, "The evolution and explosion of massive stars. II. Explosive hydrodynamics and nucleosynthesis," Astrophysical Journal Supplement Series, vol. 101, article 181, 1995.

[120] C. Y. Cardall, "Supernova neutrino challenges," Nuclear Physics B, vol. 145, no. 1-3, pp. 295-300, 2005.

[121] F. S. Kitaura, H. T. Janka, and W. Hillebrandt, "Explosions of $\mathrm{O}-\mathrm{Ne}-\mathrm{Mg}$ cores, the crab supernova, and subluminous type
II-P supernovae," Astronomy and Astrophysics, vol. 450, no. 1, pp. 345-350, 2006.

[122] H. T. Janka, B. Müller, F. S. Kitaura, and R. Buras, "Dynamics of shock propagation and nucleosynthesis conditions in $\mathrm{O}-$ $\mathrm{Ne}-\mathrm{Mg}$ core supernovae," Astronomy and Astrophysics, vol. 485, no. 1, pp. 199-208, 2008.

[123] I. Sagert, T. Fischer, M. Hempel et al., "Signals of the QCD phase transition in core-collapse supernovae," Physical Review Letters, vol. 102, no. 8, Article ID 081101, 4 pages, 2009.

[124] R. Buras, H. T. Janka, M. Rampp, and K. Kifonidis, "Twodimensional hydrodynamic core-collapse supernova simulations with spectral neutrino transport II. Models for different progenitor stars," Astronomy and Astrophysics, vol. 457, no. 1, pp. 281-308, 2006.

[125] A. Marek and H. T. Janka, "Delayed neutrino-driven supernova explosions aided by the standing accretion-shock instability," The Astrophysical Journal, vol. 694, no. 1, article 664, 2009.

[126] A. Maeder and G. Meynet, "Stellar evolution with rotation. VI. The Eddington and Omega-limits, the rotational mass loss for OB and LBV stars," Astronomy and Astrophysics, vol. 361, pp. 159-166, 2000.

[127] A. Heger, S. E. Woosley, and H. C. Spruit, "Presupernova evolution of differentially rotating massive stars including magnetic fields," The Astrophysical Journal, vol. 626, no. 1, p. 350, 2005.

[128] R. Buras, M. Rampp, H. T. Janka, and K. Kifonidis, "Twodimensional hydrodynamic core-collapse supernova simulations with spectral neutrino transport I," Astronomy and Astrophysics, vol. 447, no. 3, pp. 1049-1092, 2006.

[129] B. Mueller, H. T. Janka, and A. Marek, "General relativistic explosion models of core-collapse supernovae," ArXiv eprints.

[130] H. Dimmelmeier, J. A. Font, and E. Müller, "Relativistic simulations of rotational core collapse I. Methods, initial models, and code tests," Astronomy and Astrophysics, vol. 388, no. 3, pp. 917-935, 2002.

[131] I. Cordero-Carrión, P. Cerdá-Durán, H. Dimmelmeier, J. L. Jaramillo, J. Novak, and E. Gourgoulhon, "Improved constrained scheme for the Einstein equations: an approach to the uniqueness issue," Physical Review D, vol. 79, no. 2, Article ID 024017, 17 pages, 2009.

[132] C. D. Ott, A. Burrows, L. Dessart, and E. Livne, "Twodimensional multiangle, multigroup neutrino radiationhydrodynamic simulations of postbounce supernova cores," The Astrophysical Journal, vol. 685, no. 2, article 1069, 2008.

[133] T. D. Brandt, A. Burrows, C. D. Ott, and E. Livne, "Results from core-collapse simulations with multi-dimensional, multi-angle neutrino transport," The Astrophysical Journal Letters, vol. 728, no. 1, article 8, 2011.

[134] S. W. Bruenn, "Stellar core collapse-numerical model and infall epoch,” Astrophysical Journal Supplement Series, vol. 58, pp. 771-841, 1985.

[135] S. W. Bruenn, A. Mezzacappa, W. R. Hix et al., "2D and 3D core-collapse supernovae simulation results obtained with the CHIMERA code," ArXiv e-prints. In press.

[136] A. Burrows, E. Livne, L. Dessart, C. D. Ott, and J. Murphy, "A new mechanism for core-collapse supernova explosions," The Astrophysical Journal, vol. 640, no. 2, pp. 878-890, 2006.

[137] M. Liebendörfer, S.C. Whitehouse, and T. Fischer, "The isotropic diffusion source approximation for supernova neutrino transport," The Astrophysical Journal, vol. 698, no. 2, article 1174, 2009. 
[138] Y. Suwa, K. Kotake, T. Takiwaki, S. C. Whitehouse, M. Liebendörfer, and K. Sato, "Explosion geometry of a rotating $13 \mathrm{M}_{\text {sun }}$ star driven by the SASI-aided neutrino-heating supernova mechanism," Publications of the Astronomical Society of Japan, vol. 62, pp. L49-L53, 2010.

[139] T. Takiwaki, K. Kotake, and Y. Suwa, "Three-dimensional hydrodynamic core-collapse supernova simulations for an 11.2 Msun star with spectral neutrino transport," The Astrophysical Journal, vol. 749, no. 2, article 98, 2012.

[140] M. Liebendörfer, M. Rampp, H. T. Janka, and A. Mezzacappa, "Supernova simulations with Boltzmann neutrino transport: a comparison of methods," The Astrophysical Journal, vol. 620, no. 2, article 840, 2005.

[141] T. A. Thompson, E. Quataert, and A. Burrows, "Viscosity and rotation in core-collapse supernovae," The Astrophysical Journal, vol. 620, no. 2, article 861, 2005.

[142] T. Foglizzo and M. Tagger, "Entropic-acoustic instability in shocked accretion flows," Astronomy and Astrophysics, vol. 363, pp. 174-183, 2000.

[143] T. Foglizzo, "Entropic-acoustic instability of shocked Bondi accretion I. What does perturbed Bondi accretion sound like?" Astronomy and Astrophysics, vol. 368, pp. 311-324, 2001.

[144] F. Hanke, A. Marek, B. Mueller, and H. T. Janka, "Is strong SASI activity the key to successful neutrino-driven supernova explosions?" The Astrophysical Journal, vol. 755, no. 2, article $138,2012$.

[145] C. W. Misner and D. H. Sharp, "Relativistic equations for adiabatic, spherically symmetric gravitational collapse," Physical Review, vol. 136, no. 2, pp. B571-B576, 1964.

[146] M. M. May and R. H. White, "Hydrodynamic calculations of general-relativistic collapse," Physical Review, vol. 141, no. 4, pp. 1232-1241, 1966.

[147] R. A. Schwartz, "Gravitational collapse, neutrinos and supernovae," Annals of Physics, vol. 43, no. 1, pp. 42-73, 1967.

[148] R. W. Lindquist, "Relativistic transport theory," Annals of Physics, vol. 37, no. 3, pp. 487-518, 1966.

[149] J. R. Wilson, "A Numerical Study of Gravitational Stellar Collapse," TheAstrophysical Journal, vol. 163, article 209, 1971.

[150] K. A. van Riper, "General relativistic hydrodynamics and the adiabatic collapse of stellar cores," The Astrophysical Journal, vol. 232, pp. 558-571, 1979.

[151] K. A. van Riper and J. M. Lattimer, "Stellar core collapse. I-infall epoch,” The Astrophysical Journal, vol. 249, pp. 270289, 1981.

[152] K. A. van Riper, "Stellar core collapse. II-inner core bounce and shock propagation," The Astrophysical Journal, vol. 257, pp. 793-820, 1982.

[153] S. W. Bruenn, K. R. De Nisco, and A. Mezzacappa, "General relativistic effects in the core collapse supernova mechanism," The Astrophysical Journal Letters, vol. 560, no. 1, pp. 326-338, 2001.

[154] S. Yamada, "An implicit lagrangian code for spherically symmetric general relativistic hydrodynamics with an approximate riemann solver," The Astrophysical Journal, vol. 475, no. 2, article 720, 1997.

[155] S. Yamada, H. T. Janka, and H. Suzuki, "Neutrino transport in type II supernovae: Boltzmann solver vs. Monte Carlo method," Astronomy and Astrophysics, vol. 344, pp. 533-550, 1999.

[156] K. Sumiyoshi, S. Yamada, H. Suzuki, H. Shen, S. Chiba, and H. Toki, "Postbounce evolution of core-collapse supernovae: long-term effects of the equation of state," The Astrophysical Journal, vol. 629, no. 2, pp. 922-932, 2005.

[157] K. Sumiyoshi, S. Yamada, and H. Suzuki, "Dynamics and neutrino signal of black hole formation in nonrotating failed supernovae. I. Equation of state dependence," The Astrophysical Journal, vol. 667, pp. 382-394, 2007.

[158] K. Sumiyoshi and S. Yamada, "Neutrino transfer in three dimensions for core-collapse supernovae. I. Static configurations," The Astrophysical Journal Supplement Series, vol. 199, no. 1, article 17, 2012.

[159] A. Mezzacappa and R. A. Matzner, "Computer simulation of time-dependent, spherically symmetric spacetimes containing radiating fluids-formalism and code tests," The Astrophysical Journal, vol. 343, pp. 853-873, 1989.

[160] M. Liebendörfer, A. Mezzacappa, and F. K. Thielemann, "Conservative general relativistic radiation hydrodynamics in spherical symmetry and comoving coordinates," Physical Review D, vol. 63, no. 10, Article ID 104003, 14 pages, 2001.

[161] M. Liebendörfer, O. E. B. Messer, A. Mezzacappa, S. W. Bruenn, C. Y. Cardall, and F.-K. Thielemann, "A finite difference representation of neutrino radiation hydrodynamics in spherically symmetric general relativistic spacetime," Astrophysical Journal Supplement Series, vol. 150, no. 1, pp. 263-316, 2004.

[162] E. J. Lentz, A. Mezzacappa, O. E. Bronson Messer, M. Liebendörfer, W. R. Hix, and S. W. Bruenn, "On the requirements for realistic modeling of neutrino transport in simulations of core-collapse supernovae," The Astrophysical Journal, vol. 747, no. 1, article 73, 2012.

[163] R. Buras, M. Rampp, H. T. Janka, and K. Kifonidis, "Twodimensional hydrodynamic core-collapse supernova simulations with spectral neutrino transport," Astronomy and Astrophysics, vol. 447, no. 3, pp. 1049-1092, 2006.

[164] M. Shibata and Y. I. Sekiguchi, "Magnetohydrodynamics in full general relativity: formulation and tests," Physical Review D, vol. 72, no. 4, Article ID 044014, 24 pages, 2005.

[165] M. Shibata and Y. I. Sekiguchi, "Three-dimensional simulations of stellar core collapse in full general relativity: nonaxisymmetric dynamical instabilities," Physical Review D, vol. 71, no. 2, Article ID 024014, 32 pages, 2005.

[166] C. D. Ott, H. Dimmelmeier, A. Marek et al., “3D collapse of rotating stellar iron cores in general relativity including deleptonization and a nuclear equation of state," Physical Review Letters, vol. 98, no. 26, Article ID 261101, 4 pages, 2007.

[167] M. Liebendörfer, "A simple parameterization of the consequences of deleptonization for simulations of stellar core collapse," The Astrophysical Journal, vol. 663, pp. 1042-1051, 2005.

[168] Y. Sekiguchi, "Stellar core collapse in full general relativity with microphysics," Progress of Theoretical Physics, vol. 124, no. 2, pp. 331-379, 2010.

[169] T. Kuroda, K. Kotake, and T. Takiwaki, "Fully general relativistic simulations of core-collapse supernovae with an approximate neutrino transport," The Astrophysical Journal, vol. 755, no. 1, article 11, 2012.

[170] E. Müller, "Gravitational radiation from collapsing rotating stellar cores," Astronomy and Astrophysics, vol. 114, no. 1, pp. 53-59, 1982.

[171] R. Mönchmeyer, G. Schaefer, E. Mueller, and R. E. Kates, "Gravitational waves from the collapse of rotating stellar cores," Astronomy and Astrophysics, vol. 246, no. 2, pp. 417440, 1991. 
[172] S. Yamada and K. Sato, "Gravitational radiation from rotational collapse of a supernova core," The Astrophysical Journal, vol. 450, article 245, 1995.

[173] T. Zwerger and E. Müller, "Dynamics and gravitational wave signature of axisymmetric rotational core collapse," Astronomy and Astrophysics, vol. 320, pp. 209-227, 1997.

[174] K. Kotake, S. Yamada, and K. Sato, "Gravitational radiation from axisymmetric rotational core collapse," Physical Review $D$, vol. 68, no. 4, Article ID 044023, 7 pages, 2003.

[175] K. Kotake, S. Yamada, K. Sato, K. Sumiyoshi, H. Ono, and H. Suzuki, "Gravitational radiation from rotational core collapse: effects of magnetic fields and realistic equations of state," Physical Review D, vol. 69, no. 12, Article ID 124004, 11 pages, 2004.

[176] M. Shibata and Y. I. Sekiguchi, "Gravitational waves from axisymmetric rotating stellar core collapse to a neutron star in full general relativity," Physical Review D, vol. 69, no. 8, Article ID 084024, 16 pages, 2004.

[177] C. D. Ott, A. Burrows, E. Livne, and R. Walder, "Gravitational waves from axisymmetric, rotating stellar core collapse," The Astrophysical Journal, vol. 600, no. 2, article 834, 2004.

[178] C. D. Ott, H. Dimmelmeier, A. Marek et al., "3D collapse of rotating stellar iron cores in general relativity including deleptonization and a nuclear equation of state," Physical Review Letters, vol. 98, no. 26, Article ID 261101, 4 pages, 2007.

[179] C. D. Ott, H. Dimmelmeier, A. Marek et al., "Rotating collapse of stellar iron cores in general relativity," Classical and Quantum Gravity, vol. 24, no. 12, pp. S139-S154, 2007.

[180] H. Dimmelmeier, J. A. Font, and E. Müller, "Relativistic simulations of rotational core collapse. II. Collapse dynamics and gravitational radiation," Astronomy and Astrophysics, vol. 393, pp. 523-542, 2002.

[181] H. Dimmelmeier, C. D. Ott, H. T. Janka, A. Marek, and E. Müller, "Generic gravitational-Wave signals from the collapse of rotating stellar cores," Physical Review Letters, vol. 98, no. 25, Article ID 251101, 4 pages, 2007.

[182] H. Dimmelmeier, C. D. Ott, A. Marek, and H. T. Janka, "Gravitational wave burst signal from core collapse of rotating stars," Physical Review D, vol. 78, no. 6, Article ID 064056, 28 pages, 2008.

[183] S. Scheidegger, T. Fischer, S. C. Whitehouse, and M. Liebendörfer, "Gravitational waves from 3D MHD core collapse simulations," Astronomy and Astrophysics, vol. 490, no. 1, pp. 231-241, 2008.

[184] S. Scheidegger, R. Kaeppeli, S. C. Whitehouse, T. Fischer, and M. Liebendöerfer, "The influence of model parameters on the prediction of gravitational wave signals from stellar core collapse," Astronomy and Astrophysics, vol. 514, article A51, 23 pages, 2010.

[185] M. Liebendörfer, "A simple parameterization of the consequences of deleptonization for simulations of stellar core collapse," The Astrophysical Journal, vol. 663, pp. 1042-1051, 2005.

[186] C. D. Ott, A. Burrows, T. A. Thompson, E. Livne, and R. Walder, "The spin periods and rotational profiles of neutron stars at birth," The Astrophysical Journal, vol. 164, no. 1, article 130, 2006.

[187] K. Kotake, "Multiple physical elements to determine the gravitational-wave signatures of core-collapse supernovae," Comptes Rendus Physique. In press.

[188] A. Burrows and J. Hayes, "Pulsar recoil and gravitational radiation due to asymmetrical stellar collapse and explosion," Physical Review Letters, vol. 76, pp. 352-355, 1996.
[189] E. Müller and H. T. Janka, "Gravitational radiation from convective instabilities in type II supernova explosions," Astronomy and Astrophysics, vol. 317, pp. 140-163, 1997.

[190] E. Müller, M. Rampp, R. Buras, H. T. Janka, and D. H. Shoemaker, "Toward gravitational wave signals from realistic core-collapse supernova models ," The Astrophysical Journal, vol. 603, no. 1, pp. 221-230, 2004.

[191] M. Rampp, E. Mueller, and M. Ruffert, "Simulations of non-axisymmetric rotational core collapse," Astronomy and Astrophysics, vol. 332, pp. 969-983, 1998.

[192] C. D. Ott, H. Dimmelmeier, A. Marek et al., “3D collapse of rotating stellar iron cores in general relativity including deleptonization and a nuclear equation of state," Physical Review Letters, vol. 98, no. 26, Article ID 261101, 4 pages, 2007.

[193] C. D. Ott, A. Burrows, L. Dessart, and E. Livne, "A new mechanism for gravitational-wave emission in core-collapse supernovae," Physical Review Letters, vol. 96, no. 20, Article ID 201102, 4 pages, 2006.

[194] K. Kotake, W. Iwakami, N. Ohnishi, and S. Yamada, "Stochastic nature of gravitational waves from supernova explosions with standing accretion shock instability," The Astrophysical Journal Letters, vol. 697, no. 2, article L133, 2009.

[195] K. Kotake, N. Ohnishi, and S. Yamada, "Gravitational radiation from standing accretion shock instability in corecollapse supernovae," The Astrophysical Journal, vol. 655, no. 1, article 406, 2007.

[196] K. Kotake, W. Iwakami, N. Ohnishi, and S. Yamada, "Raytracing analysis of anisotropic neutrino radiation for estimating gravitational waves in core-collapse supernovae," The Astrophysical Journal, vol. 704, article 951, 2009.

[197] A. Marek, H. T. Janka, and E. Müller, "Equation-of-state dependent features in shock-oscillation modulated neutrino and gravitational-wave signals from supernovae," Astronomy and Astrophysics, vol. 496, no. 2, pp. 475-494, 2009.

[198] J. W. Murphy, C. D. Ott, and A. Burrows, "A model for gravitational wave emission from neutrino-driven corecollapse supernovae," ArXiv e-prints.

[199] C. D. Ott, C. Reisswig, E. Schnetter et al., "Dynamics and gravitational wave signature of collapsar formation," Physical Review Letters, vol. 106, no. 16, Article ID 161103, 4 pages, 2011.

[200] W. D. Arnett and C. Meakin, "Toward realistic progenitors of core-collapse supernovae," The Astrophysical Journal Letters, vol. 733, no. 2, article 78, 2011.

[201] R . Epstein, "The generation of gravitational radiation by escaping supernova neutrinos," The Astrophysical Journal, vol. 223, pp. 1037-1045, 1978.

[202] M. S. Turner and R. V. Wagoner, in Sources of Gravitational Radiation, L. L. Smarr, Ed., pp. 383-407, 1979.

[203] M. Favata, "The gravitational-wave memory effect," Classical and Quantum Gravity, vol. 27, no. 8, Article ID 084036, 2010.

[204] T. Hiramatsu, K. Kotake, H. Kudoh, and A. Taruya, "Gravitational wave background from neutrino-driven gamma-ray bursts," Monthly Notices of the Royal Astronomical Society, vol. 364, no. 3, pp. 1063-1068, 2005.

[205] Y. Suwa and K. Murase, "Probing the central engine of long gamma-ray bursts and hypernovae with gravitational waves and neutrinos," Physical Review D, vol. 80, no. 12, Article ID 123008, 11 pages, 2009.

[206] K. Kotake, T. Takiwaki, and S. Harikae, "Gravitational wave signatures of hyperaccreting collapsar disks," The Astrophysical Journal, vol. 755, no. 2, article 84, 2012. 
[207] Y. Suwa, T. Takiwaki, K. Kotake, and K. Sato, "Gravitational wave background from population III stars," The Astrophysical Journal Letters, vol. 665, pp. L43-L46, 2007.

[208] K. N. Yakunin, P. Marronetti, A. Mezzacappa et al., "Gravitational waves from core collapse supernovae," Classical and Quantum Gravity, vol. 27, no. 19, Article ID 194005, 2010.

[209] C. L. Fryer, D. E. Holz, and S. A. Hughes, "Gravitational waves from stellar collapse: correlations to explosion asymmetries," The Astrophysical Journal, vol. 609, pp. 288-300, 2004.

[210] K. Kotake, W. Iwakami-Nakano, and N. Ohnishi, "Effects of rotation on stochasticity of gravitational waves in the nonlinear phase of core-collapse supernovae," The Astrophysical Journal, vol. 736, no. 2, article 124, 2011.

[211] E. Müller, H. T. Janka, and A. Wongwathanarat, "Parametrized 3D models of neutrino-driven supernova explosions: neutrino emission asymmetries and gravitatio-nalwave signals," Astronomy \& Astrophysics, vol. 537, article A63, 20 pages, 2012.

[212] J. M. Blondin and A. Mezzacappa, "Pulsar spins from an instability in the accretion shock of supernovae," Nature, vol. 445, no. 7123, pp. 58-60, 2007.

[213] E. Rantsiou, A. Burrows, J. Nordhaus, and A. Almgren, "Induced rotation in three-dimensional simulations of corecollapse supernovae: implications for pulsar spins," The Astrophysical Journal, vol. 732, no. 1, article 57, 2011.

[214] T. Yamasaki and T. Foglizzo, "Effect of rotation on the stability of a stalled cylindrical shock and its consequences for core-collapse supernovae," The Astrophysical Journal, vol. 679, no. 1, pp. 607-615, 2008.

[215] K. S. Thorne, in Particle and Nuclear Astrophysics and Cosmology in the Next Millenium, E. W. Kolb and R. D. Peccei, Eds., p. 160, World Scientific, 1995.

[216] K. Kuroda and LCGT Collaboration, "Status of LCGT," Classical and Quantum Gravity, vol. 27, no. 8, Article ID 084004, 2010.

[217] S. E. A. Kawamura, "The Japanese space gravitational wave antenna-DECIGO," Classical and Quantum Gravity, vol. 23, no. 8, article S125, 2006.

[218] É. É. Flanagan and S. A. Hughes, "Measuring gravitational waves from binary black hole coalescences. II. The waves' information and its extraction, with and without templates," Physical Review D, vol. 57, no. 8, pp. 4566-4587, 1998.

[219] T. Takiwaki and K. Kotake, "Gravitational wave signatures of magnetohydrodynamically-driven core-collapse supernova explosions," The Astrophysical Journal, vol. 743, no. 1, article 30, 2011.

[220] C. D. Ott, "Probing the core-collapse supernova mechanism with gravitational waves," Classical and Quantum Gravity, vol. 26, no. 20, Article ID 204015, 2009.

[221] M. Hashimoto, "Supernova nucleosynthesis in massive stars," Progress of Theoretical Physics, vol. 94, no. 5, pp. 663-736, 1995.

[222] A. Jerkstrand, "Spectral modeling of nebular-phase supernovae," ArXiv e-prints.

[223] S. E. Woosley and R. D. Hoffman, "Co-57 and Ti-44 production in SN 1987A," The Astrophysical Journal, vol. 368, pp. L31-L34, 1991.

[224] P. Bouchet, M. M. Phillips, N. B. Suntzeff, C. Gouiffes, R. W. Hanuschik, and D. H. Wooden, "The bolometric lightcurve of supernova 1987A-part two-results from visible and infrared spectrophotometry," Astronomy and Astrophysics, vol. 245, no. 2, article 490, 1991.
[225] C. Fransson and C. Kozma, "The freeze-out phase of SN 1987A-implications for the light curve," The Astrophysical Journal, vol. 408, no. 1, pp. L25-L28, 1993.

[226] P. A. Mazzali, K. Nomoto, F. Patat, and K. Maeda, "The nebular spectra of the hypernova SN 1998bw and evidence for asymmetry," The Astrophysical Journal, vol. 559, no. 2, pp. 1047-1053, 2001.

[227] I. Maurer, A. Jerkstrand, P. A. Mazzali et al., "NERO-a postmaximum supernova radiation transport code," Monthly Notices of the Royal Astronomical Society, vol. 418, no. 3, pp. 1517-1525, 2011.

[228] A. Jerkstrand, C. Fransson, and C. Kozma, "The ${ }^{44}$ Ti-powered spectrum of SN 1987A," Astronomy and Astrophysics, vol. 530, article A45, 23 pages, 2011.

[229] G. F. Varani, W. P. S. Meikle, J. Spyromilio, and D. A. Allen, "Direct observation of radioactive cobalt decay in supernova 1987A," Monthly Notices of the Royal Astronomical Society, vol. 245, pp. 570-576, 1990.

[230] J. D. Kurfess, W. N. Johnson, R. L. Kinzer et al., "Oriented scintillation spectrometer experiment observations of Co-57 in SN 1987A," The Astrophysical Journal, vol. 399, no. 2, pp. L137-L140, 1992.

[231] A. V. Filippenko, "Optical spectra of supernovae," Annual Review of Astronomy and Astrophysics, vol. 35, pp. 309-355, 1997.

[232] N. N. Chugai, R. A. Chevalier, R. P. Kirshner, and P. M. Challis, "Hubble space telescope spectrum of SN 1987A at an age of 8 years: radioactive luminescence of cool gas," The Astrophysical Journal Letters, vol. 483, no. 2, pp. 925-940, 1997.

[233] P. Lundqvist, C. Kozma, J. Sollerman, and C. Fransson, "ISO/SWS observations of SN 1987A. II. A refined upper limit on the mass of ${ }^{44} \mathrm{Ti}$ in the ejecta of SN 1987A," Astronomy and Astrophysics, vol. 374, pp. 629-637, 2001.

[234] C.L. Fryer, G. Rockefeller, and M.S. Warren, "SNSPH: a parallel three-dimensional smoothed particle radiation hydrodynamics code," The Astrophysical Journal, vol. 643, no. 1, pp. 292-305, 2006.

[235] G. Magkotsios, F. X. Timmes, A. L. Hungerford, C. L. Fryer, P. A. Young, and M. Wiescher, "Trends in ${ }^{44} \mathrm{Ti}$ and ${ }^{56} \mathrm{Ni}$ from core-collapse supernovae," Astrophysical Journal Supplement Series, vol. 191, no. 1, article 66, 2010.

[236] F. K. Thielemann, K. Nomoto, and M. A. Hashimoto, "Corecollapse supernovae and their ejecta," The Astrophysical Journal, vol. 460, article 408, 1996.

[237] T. Rauscher, A. Heger, R. D. Hoffman, and S. E. Woosley, "Nucleosynthesis in massive stars with improved nuclear and stellar physics," The Astrophysical Journal, vol. 576, no. 1, pp. 323-348, 2002.

[238] A. L. Hungerford, C. L. Fryer, and G. Rockefeller, "Gamma rays from single-lobe supernova explosions," The Astrophysical Journal, vol. 635, no. 1, pp. 487-501, 2005.

[239] P. A. Young, C. L. Fryer, A. Hungerford et al., "Constraints on the progenitor of Cassiopeia A," The Astrophysical Journal, vol. 640, no. 2, article 891, 2006.

[240] C. C. Joggerst, A. Almgren, and S. E. Woosley, "Threedimensional simulations of rayleigh-taylor mixing in corecollapse supernovae with castro," The Astrophysical Journal, vol. 723, no. 1, pp. 353-363, 2010.

[241] S. M. Couch, Wheeler J. C., and M. Milosavljević, "Aspherical core-collapse supernovae in red supergiants powered by nonrelativistic jets," The Astrophysical Journal, vol. 696, no. 1, pp. 953-970, 2009. 
[242] N. Tominaga, "Aspherical properties of hydrodynamics and nucleosynthesis in jet-induced supernovae," The Astrophysical Journal, vol. 690, no. 1, pp. 526-536, 2009.

[243] K. Maeda and K. Nomoto, "Bipolar supernova explosions: nucleosynthesis and implications for abundances in extremely metal-poor stars," The Astrophysical Journal, vol. 598, no. 2, article 1163, 2003.

[244] A. Gawryszczak, J. Guzman, T. Plewa, and K. Kifonidis, "Non-spherical core collapse supernovae. III. Evolution towards homology and dependence on the numerical resolution," Astronomy and Astrophysics, vol. 521, article A38, 2010.

[245] J. Pruet, R. D. Hoffman, S. E. Woosley, H. T. Janka, and R. Buras, "Nucleosynthesis in early supernova winds. II. The role of neutrinos," The Astrophysical Journal, vol. 644, no. 2, pp. 1028-1039, 2006.

[246] C. Fröhlich, G. Martínez-Pinedo, M. Liebendörfer et al., "Neutrino-induced nucleosynthesis of $A>64$ nuclei: the $v p$ process," Physical Review Letters, vol. 96, no. 14, Article ID 142502, 4 pages, 2006.

[247] S. Wanajo and H. T. Janka, "The r-process in the neutrinodriven wind from a black-hole torus," The Astrophysical Journal, vol. 746, no. 2, article 180, 2012.

[248] S. I. Fujimoto, K. Kotake, M. A. Hashimoto, M. Ono, and N. Ohnishi, "Explosive nucleosynthesis in the neutrino-driven aspherical supernova explosion of a non-rotating $15 \mathrm{M}_{\text {sun }}$ star with solar metallicity," The Astrophysical Journal, vol. 738, no. 1, article 61, 2011.

[249] R. H. Cyburt, J. Ellis, B. D. Fields, F. Luo, K. A. Olive, and V. C. Spanos, "Nuclear reaction uncertainties, massive gravitino decays and the cosmological lithium problem," Journal of Cosmology and Astroparticle Physics, vol. 2010, article 32, 2010.

[250] J. Schwab, P. Podsiadlowski, and S. Rappaport, "Further evidence for the bimodal distribution of neutron star masses," The Astrophysical Journal, vol. 719, pp. 722-727, 2010.

[251] M. Kimura, H. Tsunemi, S. Katsuda, and H. Uchida, "Suzaku observations across the cygnus loop from the NorthEastern to the SouthWestern rim," Publications of the Astronomical Society of Japan, vol. 61, pp. S137-S145, 2009.

[252] H. Uchida, H. Tsunemi, S. Katsuda, M. Kimura, H. Kosugi, and H. Takahashi, "Abundance inhomogeneity in the Northern rim of the cygnus loop," Publications of the Astronomical Society of Japan, vol. 61, no. 3, pp. 503-510, 2009.

[253] T. Shigeyama, K. Nomoto, and M. Hashimoto, "Hydrodynamical models and the light curve of supernova 1987A in the large magellanic cloud," Astronomy and Astrophysics, vol. 196, no. 1-2, pp. 141-151, 1988.

[254] M. Renaud, J. Vink, A. Decourchelle et al., "The signature of ${ }^{44} \mathrm{Ti}$ in Cassiopeia A revealed by IBIS/ISGRI on INTEGRAL," The Astrophysical Journal Letters, vol. 647, no. 1, article L41, 2006.

[255] J. M. LeBlanc and J. R. Wilson, "A numerical example of the collapse of a rotating magnetized star," The Astrophysical Journal, vol. 161, article 541, 1970.

[256] G. S. Bisnovatyi-Kogan, Y. P. Popov, and A. A. Samochin, "The magnetohydrodynamic rotational model of supernova explosion," Astrophysics and Space Science, vol. 41, no. 2, pp. 287-320, 1976.

[257] E. Müller and W. Hillebrandt, "A magnetohydrodynamical supernova model," Astronomy and Astrophysics, vol. 80, no. 2, pp. 147-154, 1979.

[258] E. M. D. Symbalisty, "Magnetorotational iron core collapse," The Astrophysical Journal, vol. 285, pp. 729-746, 1984.
[259] N. V. Ardeljan, G. S. Bisnovatyi-Kogan, and S. G. Moiseenko, "Nonstationary magnetorotational processes in a rotating magnetized cloud," Astronomy and Astrophysics, vol. 355, no. 3, pp. 1181-1190, 2000.

[260] S. Yamada and H. Sawai, "Numerical study on the rotational collapse of strongly magnetized cores of massive stars," The Astrophysical Journal, vol. 608, no. 2, article 907, 2004.

[261] K. Kotake, H. Sawai, S. Yamada, and K. Sato, "Magnetorotational effects on anisotropic neutrino emission and convection in core collapse supernovae," The Astrophysical Journal, vol. 608, no. 1, article 391, 2004.

[262] K. Kotake, S. Yamada, and K. Sato, "North-South neutrino heating asymmetry in strongly magnetized and rotating stellar cores," The Astrophysical Journal, vol. 618, pp. 474-484, 2005.

[263] M. Obergaulinger, M. A. Aloy, H. Dimmelmeier, and E. Müller, "Axisymmetric simulations of magnetorotational core collapse: approximate inclusion of general relativistic effects," Astronomy and Astrophysics, vol. 457, no. 1, pp. 209222, 2006.

[264] M. Obergaulinger, M. A. Aloy, and E. Müller, "Axisymmetric simulations of magneto-rotational core collapse: dynamics and gravitational wave signal," Astronomy and Astrophysics, vol. 450, no. 3, pp. 1107-1134, 2006.

[265] P. Cerdá-Durán, J. A. Font, and H. Dimmelmeier, “General relativistic simulations of passive-magneto-rotational core collapse with microphysics," Astronomy and Astrophysics, vol. 474, no. 1, pp. 169-191, 2007.

[266] Y. Suwa, T. Takiwaki, K. Kotake, and K. Sato, "Magnetorotational collapse of population III stars," Publications of the Astronomical Society of Japan, vol. 59, pp. 771-785, 2007.

[267] T. Takiwaki, K. Kotake, S. Nagataki, and K. Sato, "Magnetodriven shock waves in core-collapse supernovae," The Astrophysical Journal, vol. 616, no. 2, article 1086, 2004.

[268] T. Takiwaki, K. Kotake, and K. Sato, "Special relativistic simulations of magnetically-dominated jets in collapsing massive stars," The Astrophysical Journal, vol. 691, no. 2, article 1360, 2009.

[269] S. Scheidegger, R. Kaeppeli, S. C. Whitehouse, T. Fischer, and M. Liebendoerfer, "The influence of model parameters on the prediction of gravitational wave signals from stellar core collapse," . In press, http://arxiv.org/abs/1001.1570.

[270] M. Obergaulinger and H. T. Janka, "Magnetic field amplification in collapsing, non-rotating stellar cores," . In press, http://arxiv.org/abs/1101.1198.

[271] R. C. Duncan and C. Thompson, "Formation of very strongly magnetized neutron stars-implications for gamma-ray bursts," The Astrophysical Journal Letters, vol. 392, no. 1, pp. L9-L13, 1992.

[272] J.M. Lattimer and M. Prakash, "Neutron star observations: prognosis for equation of state constraints," Physics Reports, vol. 442, no. 1-6, pp. 109-165, 2007.

[273] L. Dessart, A. Burrows, E. Livne, and C. D. Ott, "The proto-neutron star phase of the collapsar model and the route to long-soft gamma-ray bursts and hypernovae," The Astrophysical Journal Letters, vol. 673, no. 1, pp. L43-L46, 2008.

[274] S. E. Woosley and A. Heger, "The progenitor stars of gammaray bursts," The Astrophysical Journal, vol. 637, no. 2, article 914, 2006.

[275] S. C. Yoon and N. Langer, "Evolution of rapidly rotating metal-poor massive stars towards gamma-ray bursts," Astronomy and Astrophysics, vol. 443, no. 2, pp. 643-648, 2005. 
[276] D. L. Meier, R. I. Epstein, W. D. Arnett, and D. N. Schramm, "Magnetohydrodynamic phenomena in collapsing stellar cores," The Astrophysical Journal, vol. 204, pp. 869-878, 1976.

[277] S. Akiyama, J. C. Wheeler, D. L. Meier, and I. Lichtenstadt, "The magnetorotational instability in core-collapse supernova explosions," Astrophysical Journal Letters, vol. 584, no. 2, pp. 954-970, 2003.

[278] J. F. Hawley, C. F. Gammie, and S. A. Balbus, "Local threedimensional magnetohydrodynamic simulations of accretion disks," The Astrophysical Journal, vol. 440, article 742, 1995.

[279] Y. Masada, T. Sano, and H. Takabe, "Nonaxisymmetric magnetorotational instability in proto-neutron stars," The Astrophysical Journal, vol. 641, no. 1, pp. 447-457, 2006.

[280] M. Shibata, Y. T. Liu, S. L. Shapiro, and B. C. Stephens, "Magnetorotational collapse of massive stellar cores to neutron stars: simulations in full general relativity," Physical Review D, vol. 74, no. 10, Article ID 104026, 28 pages, 2006.

[281] Z. B. Etienne, Y. T. Liu, and S. L. Shapiro, "General relativistic simulations of slowly and differentially rotating magnetized neutron stars," Physical Review D, vol. 74, no. 4, Article ID 044030, 2006.

[282] M. Obergaulinger, P. Cerdá-Durán, E. Müller, and M. A. Aloy, "Semi-global simulations of the magneto-rotational instability in core collapse supernovae," Astronomy and Astrophysics, vol. 498, no. 1, pp. 241-271, 2009.

[283] W. Zhang, A. MacFadyen, and P. Wang, "Three-dimensional relativistic MHD simulations of the Kelvin-Helmholtz instability: magnetic field amplification by a turbulent dynamo," The Astrophysical Journal Letters, vol. 692, no. 1, article L40, 2009.

[284] T. Sano, S. Inutsuka, and S. M. Miyama, "A saturation mechanism of magnetorotational instability due to ohmic dissipation," The Astrophysical Journal, vol. 506, no. 1, pp. L57-L60, 1998.

[285] B. van Leer, "Towards the ultimate conservative difference scheme. V. A second-order sequel to Godunov's method," Journal of Computational Physics, vol. 32, no. 1, pp. 101-136, 1979.

[286] D. A. Clarke, "A consistent method of characteristics for multidimensional magnetohydrodynamics," The Astrophysical Journal Letters, vol. 457, no. 1, pp. 291-320, 1996.

[287] C. R. Evans and J. F. Hawley, "Simulation of magnetohydrodynamic flows- a constrained transport method," The Astrophysical Journal, vol. 332, pp. 659-677, 1988.

[288] J. M. Stone and M. L. Norman, "ZEUS-2D: a radiation magnetohydrodynamics code for astrophysical flows in two space dimensions. I- the hydrodynamic algorithms and tests," Astrophysical Journal Supplement Series, vol. 80, no. 2, pp. 753-790, 1992.

[289] M. E. Pessah and J. Goodman, "On the saturation of the magnetorotational instability via parasitic modes," The Astrophysical Journal, vol. 698, no. 1, pp. L72-L76, 2009.

[290] P. Longaretti and G. Lesur, "MRI-driven turbulent transport: the role of dissipation, channel modes and their parasites," Astronomy and Astrophysics, vol. 516, article A51, 2010.

[291] J. F. Hawley and S. A. Balbus, "A powerful local shear instability in weakly magnetized disks. III-long-term evolution in a shearing sheet," The Astrophysical Journal, vol. 400, no. 2, pp. 595-609, 1992.

[292] J. Goodman and G. Xu, "Parasitic instabilities in magnetized, differentially rotating disks," The Astrophysical Journal, vol. 432, no. 1, pp. 213-223, 1994.

[293] T. Sano and S. Inutsuka, "Saturation and thermalization of the magnetorotational instability: recurrent channel flows and reconnections," The Astrophysical Journal, vol. 561, no. 2, pp. L179-182, 2001.

[294] G. Bodo, A. Mignone, F. Cattaneo, P. Rossi, and A. Ferrari, "Aspect ratio dependence in magnetorotational instability shearing box simulations," Astronomy and Astrophysics, vol. 487, no. 1, pp. 1-5, 2008.

[295] T. Sano, S. Inutsuka, N. J. Turner, and J. M. Stone, "Angular momentum transport by magnetohydrodynamic turbulence in accretion disks: gas pressure dependence of the saturation level of the magnetorotational instability," The Astrophysical Journal, vol. 605, no. 1, pp. 321-339, 2004.

[296] M. E. Pessah and C. Chan, "Viscous, resistive magnetorotational modes," The Astrophysical Journal, vol. 684, no. 1, pp. 498-514, 2008.

[297] Y. Masada, T. Takiwaki, K. Kotake, and T. Sano, "Local simulations of the magneto-rotational instability in corecollapse supernovae," The Astrophysical Journal. In press, http://arxiv.org/abs/1209.2360.

[298] A. I. MacFadyen and S. E. Woosley, "Collapsars: gamma-ray bursts and explosions in 'failed supernovae," The Astrophysical Journal, vol. 524, no. 1, article 262, 1999.

[299] A. I. MacFadyen, S. E. Woosley, and A. Heger, "Supernovae, jets, and collapsars," The Astrophysical Journal, vol. 550, no. 1, pp. 410-425, 2001.

[300] S. Harikae, T. Takiwaki, and K. Kotake, "Long-term evolution of slowly rotating collapsar in special relativistic magnetohydrodynamics," The Astrophysical Journal, vol. 704, no. 1, article 354, 2009.

[301] S. Harikae, K. Kotake, and T. Takiwaki, "Neutrino pair annihilation in collapsars: a ray-tracing method in special relativity," The Astrophysical Journal, vol. 713, no. 1, pp. 304$317,2010$.

[302] P. Meszaros, "Gamma-ray bursts," Reports on Progress in Physics, vol. 69, no. 8, article 2259, 2006.

[303] K. S. Thorne, "Multipole expansions of gravitational radiation," Reviews of Modern Physics, vol. 52, no. 2, pp. 299-339, 1980.

[304] A. Weinstein, "Advanced LIGO optical configuration and prototyping effort," Classical and Quantum Gravity, vol. 19, article 1575, 2002.

[305] H. Kudoh, A. Taruya, T. Hiramatsu, and Y. Himemoto, "Detecting a gravitational-wave background with nextgeneration space interferometers," Physical Review D, vol. 73, no. 6, Article ID 064006, 16 pages, 2006.

[306] S. Kawagoe, T. Takiwaki, and K. Kotake, "Neutrino oscillations in magnetically driven supernova explosions," Journal of Cosmology and Astroparticle Physics, vol. 2009, no. 9, article 33, 2009.

[307] J. Engel, G. C. McLaughlin, and C. Volpe, "What can be learned with a lead-based supernova-neutrino detector?" Physical Review D, vol. 67, no. 1, Article ID 013005, 2003.

[308] D. Autiero, J. Äystö, A. Badertscher et al., "Large underground, liquid based detectors for astro-particle physics in Europe: scientific case and prospects," Journal of Cosmology and Astroparticle Physics, vol. 2007, no. 11, article 011, 2007.

[309] K. Scholberg, "Future supernova neutrino detectors," Journal of Physics Conference Series, vol. 203, no. 1, Article ID 012079, 2010.

[310] M. Kachelrieß, R. Tomas, R. Buras, H. T. Janka, A. Marek, and M. Rampp, "Exploiting the neutronization burst of a galactic supernova," Physical Review D, vol. 71, no. 6, Article ID 063003, 14 pages, 2005. 
[311] J. F. Beacom and M. R. Vagins, "Antineutrino spectroscopy with large water Čerenkov detectors," Physical Review Letters, vol. 93, no. 17, Article ID 171101, 4 pages, 2004.

[312] L. Hüdepohl, B. Müller, H. T. Janka, A. Marek, and G. G. Raffelt, "Neutrino signal of electron-capture supernovae from core collapse to cooling," Physical Review Letters, vol. 104, no. 25, Article ID 251101, 2010.

[313] T. Fischer, S. C. Whitehouse, A. Mezzacappa, F. K. Thielemann, and M. Liebendörfer, "Protoneutron star evolution and the neutrino-driven wind in general relativistic neutrino radiation hydrodynamics simulations," Astronomy and Astrophysics, vol. 517, article A80, 25 pages, 2010.

[314] E. O'Connor and C. D. Ott, "Black hole formation in failing core-collapse supernovae," The Astrophysical Journal, vol. 730, no. 2, article 70, 2011.

[315] K. Takahashi, K. Sato, A. Burrows, and T. A. Thompson, "Supernova neutrinos, neutrino oscillations, and the mass of the progenitor star," Physical Review D, vol. 68, no. 11, Article ID 113009, 8 pages, 2003.

[316] C. Lunardini and A.Y. Smirnov, "Supernova neutrinos: Earth matter effects and neutrino mass spectrum," Nuclear Physics $B$, vol. 616, no. 1-2, pp. 307-348, 2001.

[317] A. S. Dighe, M. Kachelrieß, G. G. Raffelt, and R. Tomàs, "Signatures of supernova neutrino oscillations in the Earth mantle and core," Journal of Cosmology and Astroparticle Physics, vol. 2004, article 4, 2004.

[318] R. C. Schirato and G. M. Fuller, "Connection between supernova shocks, flavor transformation, and the neutrino signal," in press, http://arxiv.org/abs/astro-ph/0205390.

[319] C. Lunardini and A. Y. Smirnov, "Probing the neutrino mass hierarchy and the 13-mixing with supernovae," Journal of Cosmology and Astroparticle Physics, vol. 2003, no. 6, article 9, 2003.

[320] R. Tomas, M. Kachelrieß, G. Raffelt, A. Dighe, H. T. Janka, and L. Scheck, "Neutrino signatures of supernova forward and reverse shock propagation," Journal of Cosmology and Astroparticle Physics, vol. 9, article 15, 2004.

[321] B. Dasgupta and A. Dighe, "Phase effects in neutrino conversions during a supernova shock wave," Physical Review D, vol. 75, no. 9, Article ID 093002, 10 pages, 2007.

[322] C. Lunardini and O. L. G. Peres, "Upper limits on the diffuse supernova neutrino flux from the SuperKamiokande data," Journal of Cosmology and Astroparticle Physics, vol. 8, article 33, 2008.

[323] A. S. Dighe and A. Y. Smirnov, "Identifying the neutrino mass spectrum from a supernova neutrino burst," Physical Review D, vol. 62, no. 3, Article ID 033007, 24 pages, 2000.

[324] K. Takahashi, K. Sato, H. E. Dalhed, and J. R. Wilson, "Shock propagation and neutrino oscillation in supernova," Astroparticle Physics, vol. 20, no. 2, pp. 189-193, 2003.

[325] G. L. Fogli, E. Lisi, A. Mirizzi, and D. Montanino, "Probing supernova shock waves and neutrino flavour transitions in next-generation water Cherenkov detectors," Journal of Cosmology and Astroparticle Physics, vol. 4, article 2, 2005.

[326] S. Chakraborty, T. Fischer, A. Mirizzi, N. Saviano, and R. Tomas, "No collective neutrino flavor conversions during the supernova accretion phase," Physical Review Letters, vol. 107, no. 15, Article ID 151101, 4 pages, 2011.

[327] B. Dasgupta, E. P. O'Connor, and C. D. Ott, “The role of collective neutrino flavor oscillations in core-collapse supernova shock revival," Physical Review D, vol. 85, no. 6, Article ID 065008, 16 pages, 2012.
[328] Y. Suwa, K. Kotake, T. Takiwaki, M. Liebendoerfer, and K. Sato, "Impacts of collective neutrino oscillations on corecollapse supernova explosions," The Astrophysical Journal, vol. 738, no. 2, article 165, 2011.

[329] O. Pejcha, B. Dasgupta, and T. A. Thompson, "Effect of collective neutrino oscillations on the neutrino mechanism of core-collapse supernovae," in press, http://arxiv.org/ abs/1106.5718.

[330] G. G. Raffelt, Stars As Laboratories for Fundamental Physics: The Astrophysics of Neutrinos, Axions, and Other Weakly Interacting Particles, University of Chicago Press, 1996.

[331] J. Schechter and J. W. F. Valle, "Majorana neutrinos and magnetic fields," Physical Review D, vol. 24, no. 7, pp. 1883$1889,1981$.

[332] C. S. Lim and W. J. Marciano, "Resonant spin-flavor precession of solar and supernova neutrinos," Physical Review $D$, vol. 37, no. 6, pp. 1368-1373, 1988.

[333] E. K. Akhmedov and M. Y. Khlopov, "Resonant amplification of neutrino oscillations in longitudinal magnetic field," Modern Physics Letters A, vol. 3, no. 5, pp. 451-457, 1988.

[334] S. Ando and K. Sato, "Resonant spin-flavor conversion of supernova neutrinos: dependence on presupernova models and future prospects," Physical Review D, vol. 68, no. 2, Article ID 023003, 9 pages, 2003.

[335] T. Totani, K. Sato, H. E. Dalhed, and J. R. Wilson, "Future detection of supernova neutrino burst and explosion mechanism," The Astrophysical Journal, vol. 496, no. 1, article 216, 1998.

[336] C. Lunardini, B. Müller, and H. T Janka, "Neutrino oscillation signatures of oxygen-neon-magnesium supernovae," Physical Review D, vol. 78, no. 2, Article ID 023016, 13 pages, 2008.

[337] J. F. Cherry, M. R. Wu, J. Carlson, H. Duan, G. M. Fuller, and Y. Z. Qian, "Density fluctuation effects on collective neutrino oscillations in O-Ne-Mg core-collapse supernovae," Physical Review D, vol. 84, no. 10, Article ID 105034, 12 pages, 2011.

[338] S. Sarikas, G. G. Raffelt, L. Hüdepohl, and H. T. Janka, "Suppression of self-induced flavor conversion in the supernova accretion phase," Physical Review Letters, vol. 108, no. 6, Article ID 061101, 4 pages, 2012.

[339] A. Friedland and A. Gruzinov, "Neutrino signatures of supernova turbulence," Astrophysics. In press, http://arxiv.org/abs/ astro-ph/0607244.

[340] S. Choubey, N. P. Harries, and G. G. Ross, "Turbulent supernova shock waves and the sterile neutrino signature in megaton water detectors," Physical Review D, vol. 76, no. 7, Article ID 073013, 20 pages, 2007.

[341] G. Reid, J. Adams, and S. Seunarine, "Collective neutrino oscillations in turbulent backgrounds," Physical Review D, vol. 84, no. 8, Article ID 085023, 32 pages, 2011.

[342] B. Dasgupta, A. Dighe, A. Mirizzi, and G. Raffelt, "Collective neutrino oscillations in nonspherical geometry," Physical Review D, vol. 78, no. 3, Article ID 033014, 14 pages, 2008.

[343] T. Lund, A. Marek, C. Lunardini, H. T. Janka, and G. Raffelt, "Fast time variations of supernova neutrino fluxes and their detectability," Physical Review D, vol. 82, no. 6, Article ID 063007, 13 pages, 2010.

[344] K. Kotake, S. Yamada, and K. Sato, "Anisotropic neutrino radiation in rotational core collapse," The Astrophysical Journal, vol. 595, no. 1, article 304, 2003.

[345] H. T. Janka and R. Mönchmeyer, "Anisotropic neutrino emission from rotating protoneutron stars," Astronomy and Astrophysics, vol. 209, no. 1-2, pp. L5-L8, 1989. 
[346] G. Fogli, E. Lisi, A. Marrone, and A. Mirizzi, "Collective neutrino flavor transitions in supernovae and the role of trajectory averaging," Journal of Cosmology and Astroparticle Physics, vol. 2007, article 10, 2007.

[347] J.M. Lattimer and F.D. Swesty, "A generalized equation of state for hot, dense matter," Nuclear Physics A, vol. 535, no. 2, pp. 331-376, 1991.

[348] S. Shlomo, V. M. Kolomietz, and G. Colo, "Deducing the nuclear-matter incompressibilty coefficient from data on isoscalar compression modes," European Physical Journal A, vol. 30, no. 1, pp. 23-30, 2006.

[349] P. B. Demorest, T. Pennucci, S. M. Ransom, M. S. E. Roberts, and J. W. T. Hessels, "A two-solar-mass neutron star measured using Shapiro delay," Nature, vol. 467, no. 7319, pp. 1081-1083, 2010.

[350] K. Kiuchi and K. Kotake, "Equilibrium configurations of strongly magnetized neutron stars with realistic equations of state," Monthly Notices of the Royal Astronomical Society, vol. 385, no. 3, pp. 1327-1347, 2008.

[351] T. Fischer, I. Sagert, G. Pagliara et al., "Core-collapse supernova explosions triggered by a quark-hadron phase transition during the early post-bounce phase," Astrophysical Journal Supplement Series, vol. 194, article 39, 2011.

[352] T. K. Suzuki, K. Sumiyoshi, and S. Yamada, "Alfven wavedriven supernova explosion," The Astrophysical Journal, vol. 678, no. 2, pp. 1200-1206, 2008.

[353] B. Mueller, A. Marek, H. T. Janka, and H. Dimmelmeier, "General relativistic explosion models of core-collapse supernovae," ArXiv e-prints.

[354] J. Guilet, J. Sato, and T. Foglizzo, "The saturation of sasi by parasitic instabilities," The Astrophysical Journal, vol. 713, no. 2, article 1350, 2010.

[355] J. W. Murphy and C. Meakin, "A global turbulence model for neutrino-driven convection in core-collapse supernovae," The Astrophysical Journal, vol. 742, no. 2, article 74, 2011.

[356] M. Hempel and J. Schaffner-Bielich, "A statistical model for a complete supernova equation of state," Nuclear Physics A, vol. 837, no. 3-4, pp. 210-254, 2010.

[357] S. Furusawa, S. Yamada, K. Sumiyoshi, and H. Suzuki, "A new baryonic equation of state at sub-nuclear densities for corecollapse simulations," The Astrophysical Journal, vol. 738, no. 2, article 178, 2011.

[358] G. Shen, C. J. Horowitz, and E. O'Connor, "Second relativistic mean field and virial equation of state for astrophysical simulations," Physical Review C, vol. 83, no. 6, Article ID 065808, 11 pages, 2011.

[359] K. Sumiyoshi, S. Yamada, H. Suzuki, and S. Chiba, "Neutrino signals from the formation of a black hole: a probe of the equation of state of dense matter," Physical Review Letters, vol. 97, no. 9, Article ID 091101, 4 pages, 2006.

[360] K. Nakazato, K. Sumiyoshi, H. Suzuki, and S. Yamada, "Exploring hadron physics in black hole formations: a new promising target of neutrino astronomy," Physical Review D, vol. 81, no. 8, Article ID 083009, 6 pages, 2010.

[361] K. Nakazato, K. Sumiyoshi, and S. Yamada, "Impact of quarks and pions on dynamics and neutrino signal of black hole formation in non-rotating stellar core collapse," The Astrophysical Journal, vol. 721, no. 2, article 1284, 2010.

[362] K. Fukushima and T. Hatsuda, "The phase diagram of dense QCD," Reports on Progress in Physics, vol. 74, no. 1, Article ID 014001, 2011.

[363] I. Leonor, L. Cadonati, E. Coccia et al., "Searching for prompt signatures of nearby core-collapse supernovae by a joint analysis of neutrino and gravitational-wave data," ArXiv eprints.

[364] S. Nishimura, K. Kotake, M. A. Hashimoto et al., " $r$-Process nucleosynthesis in magnetohydrodynamic jet explosions of core-collapse supernovae," The Astrophysical Journal, vol. 642, pp. 410-419, 2006.

[365] S. I. Fujimoto, M. A. Hashimoto, K. Kotake, and S. Yamada, "Heavy element nucleosynthesis in a collapsar," The Astrophysical Journal, vol. 656, no. 1, pp. 382-392, 2007.

[366] J. C. Wheeler, I. Yi, P. Hoflich, and L. Wang, "Asymmetric supernovae, pulsars, magnetars, and gamma-ray bursts," The Astrophysical Journal, vol. 537, no. 2, article 810, 2000.

[367] T. A. Thompson, P. Chang, and E. Quataert, "Magnetar spindown, hyperenergetic supernovae, and gamma-ray bursts," The Astrophysical Journal, vol. 611, no. 1, pp. 380-393, 2004.

[368] D. A. Uzdensky and A. I. MacFadyen, "Magnetar-driven magnetic tower as a model for gamma-ray bursts and asymmetric supernovae," The Astrophysical Journal, vol. 669, pp. 546-560, 2007.

[369] Z. G. Dai and T. Lu, "Gamma-ray burst afterglows and evolution of postburst fireballs with energy injection from strongly magnetic millisecond pulsars," Astronomy and Astrophysics, vol. 333, pp. L87-L90, 1998.

[370] N. Bucciantini, E. Quataert, J. Arons, B. D. Metzger, and T. A. Thompson, "Relativistic jets and long-duration gammaray bursts from the birth of magnetars," Monthly Notices of the Royal Astronomical Society, vol. 383, no. 1, pp. L25-L29, 2007.

[371] B. D. Metzger, D. Giannios, T. A. Thompson, N. Bucciantini, and E. Quataert, "The protomagnetar model for gamma-ray bursts," Monthly Notices of the Royal Astronomical Society, vol. 413, no. 3, pp. 2031-3056, 2011.

[372] K. Kotake, K. Sumiyoshi, S. Yamada et al., "Core-collapse supernovae as supercomputing science: a status report toward 6D simulations with exact Boltzmann neutrino transport in full general relativity," Progress of Theoretical and Experimental Physics, vol. 2012, no. 1, Article ID 01A301, 2012. 

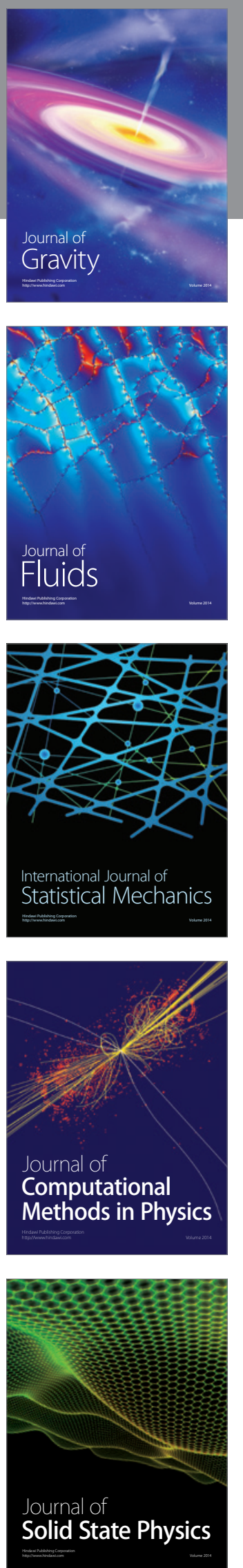

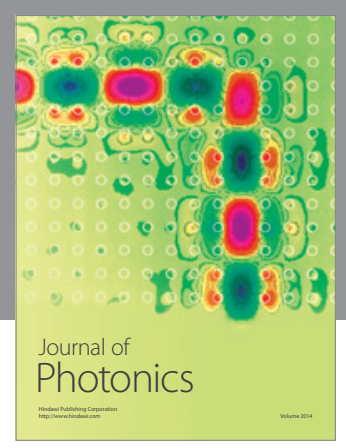

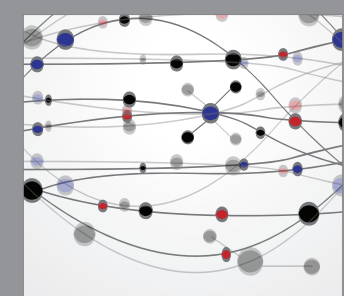

The Scientific World Journal
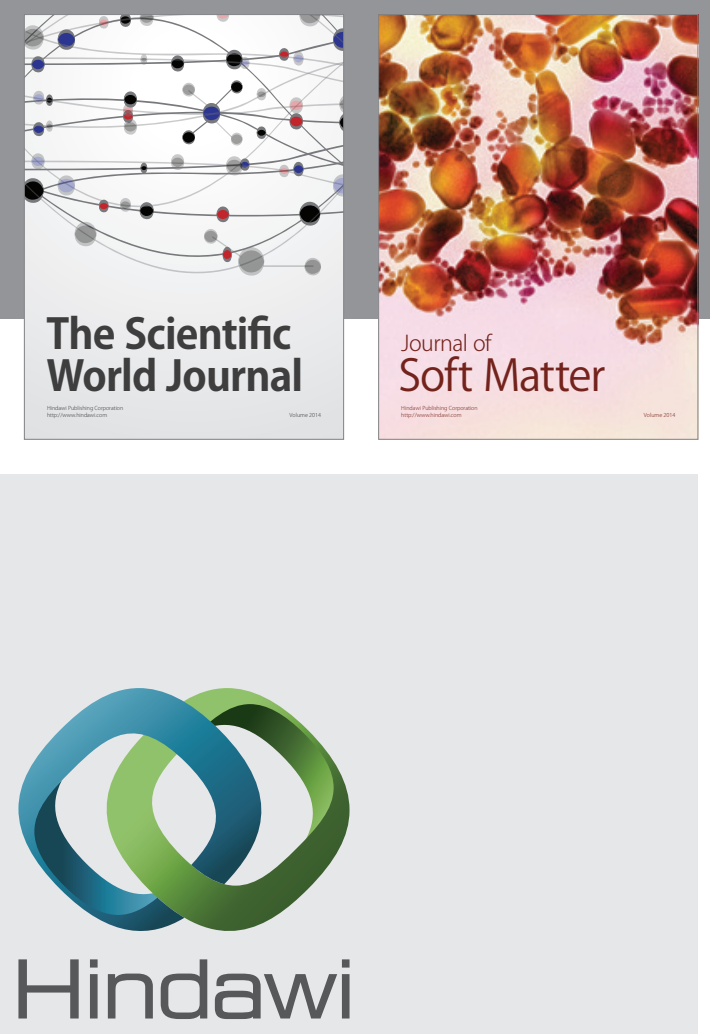

Submit your manuscripts at

http://www.hindawi.com
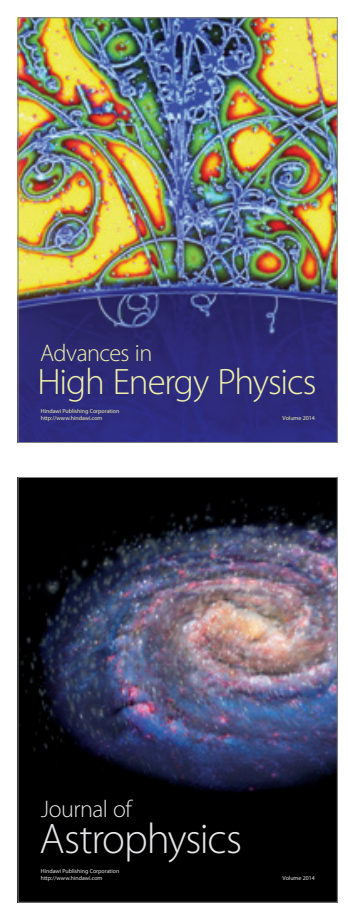
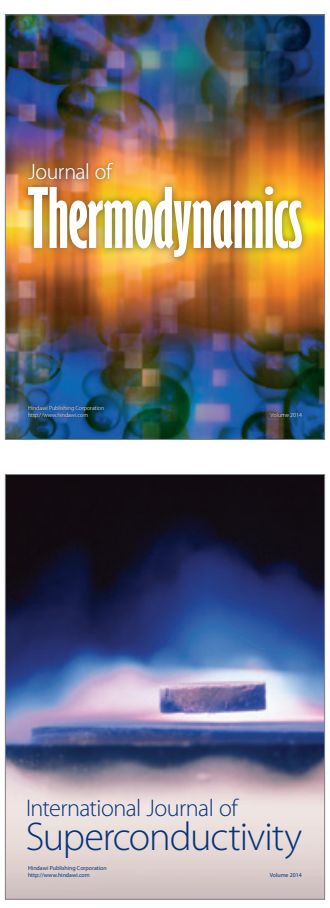
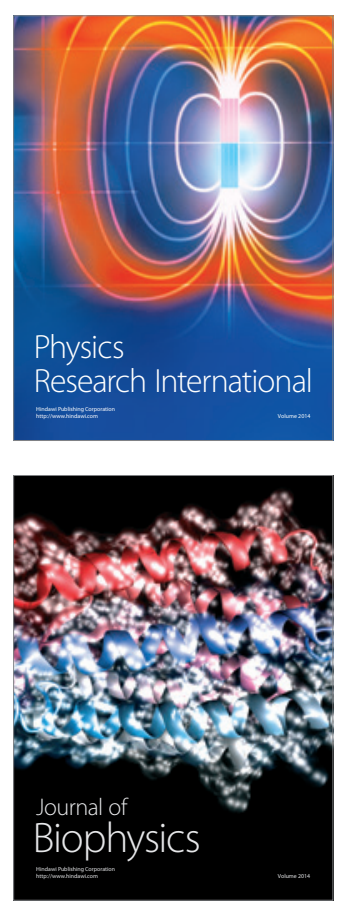
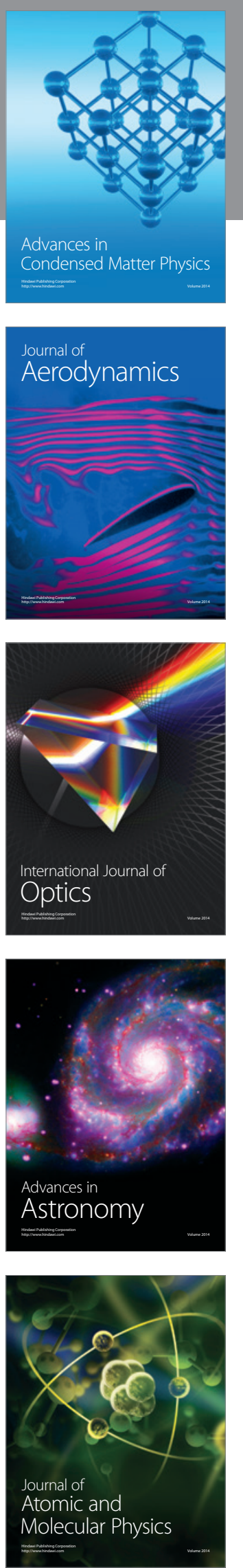